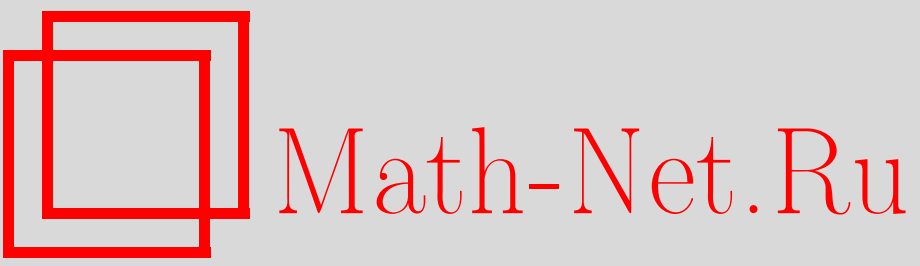

А. И. Штерн, Почти периодические функции и представления в локально выпуклых пространствах, УМН, 2005, том 60, выпуск 3, 97-168

DOI: https://doi.org/10.4213/rm1430

Использование Общероссийского математического портала Math-Net.Ru подразумевает, что вы прочитали и согласны с пользовательским соглашением

http://www.mathnet.ru/rus/agreement

Параметры загрузки:

IP: 54.166 .219 .16

26 апреля 2023 г., 13:18:25 


\title{
ПОЧТИ ПЕРИОДИЧЕСКИЕ ФУНКЦИИ И ПРЕДСТАВЛЕНИЯ В ЛОКАЛЬНО ВЫПУКЛЫХ ПРОСТРАНСТВАХ
}

\author{
А. И. ШтеРн
}

Рассматриваются свойства различных классов почти периодических функций со значениями в локально выпуклых пространствах и почти периодических представлений в локально выпуклых пространствах. Хорошо известный критерий почти периодичности слабо почти периодических представлений групп в банаховых пространствах (в терминах скалярной почти периодичности) распространяется на случай слабо непрерывных слабо почти периодических представлений в бочечных пространствах, в которых слабо замкнутые вьпуклые оболочки слабо компактных множеств слабо компактны. Указаны приложения этого результата и дан обзор современного состояния ряда других классических задач теории почти периодических функций (применительно к почти периодическим функциям со значениями в локально выпуклых пространствах) и современных направлений исследований, связанных с почти периодическими функциями на группах и конечномерными унитарными представлениями групп. В частности, рассматриваются задачи о разложении слабо почти периодических представлений и о характеризации различных классов почти периодических функций (включая критерии почти периодичности), вопросы существования среднего значения, условия счетности спектра скалярно почти периодических функций, теоремы об интеграле и о разностях почти периодических функций, а также другие связи между сильной, скалярной и слабой почти периодичностью для функций со значениями в локально выпуклых пространствах.

Библиографояи: 237 названий.

\section{СОДЕРЖАНИЕ}

$\S 1$. Введение. Основные определения. Обозначения и терминология .....

$\S 2$. Основные факты теории де Лю-Гликсберга . . . . . . . . . . . . . . . . . . .

$\S 3$. Аналог разложения де Лю-Гликсберга для слабо почти периодических представлений в бочечных локально выпуклых пространствах

$\S 4$. Связь мехду почти периодичностью, слабой почти периодичностью и скалярной почти периодичностью ........................ 116

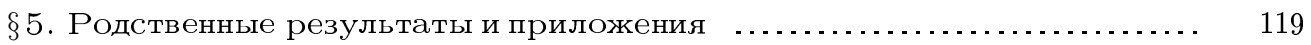

5.1. Критерии почти периодичности и слабой почти периодичности ........ 119

5.2. Среднее на слабо почти периодических функциях ................. . . 120

5.3. Условия счетности спектра скалярно почти периодической функции ... 121

5.4. Слабая почти периодичность функций и представлений … . . . . . . . . 123

5.5. Почти периодичность функций с почти периодическими разностями и почти периодичность неопределенного интеграла

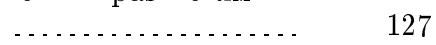




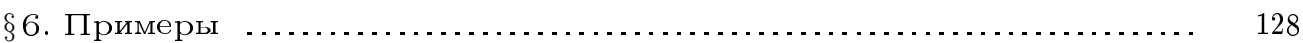

$\S 7$. Другие результаты, связанные с классическими задачами теории почти периодических функций . .............................. 129

7.1. Непосредственные следствия теоремы разложения ................ 129

7.2. Дополнительные сведения о почти периодических функциях и представлениях

7.3. Свойства почти периодических функций: существование среднего значения, спектр, теоремы о разностях и об интеграле, условия компактности, теорема Дини, свойства диссипативных функций ...................

7.4. Обобщения почти периодических функций и топологическая динамика

$\S 8$. Другие направления исследований, связанные с почти периодичес-

кими функциями

8.1. Компактификация Бора, [MAP] группы и [FIR] группы _. . . . . . . . . . . 143

8.2. Геометрия групповых алгебр. Свойства Радона-Никодима, ДанфордаПеттиса, Асплунда, Шура ...............................

8.3. Свойства слабо почти периодических компактификаций групп и полу-

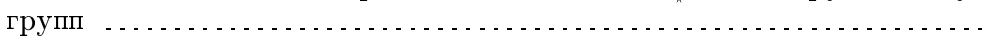

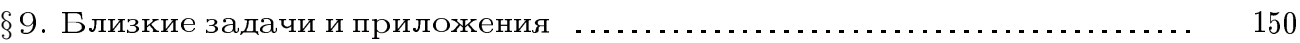

9.1. Геометрия банаховых и локально выпуклых пространств . ........... 150

9.2. Приложения слабо почти периодических функций и представлений к те-

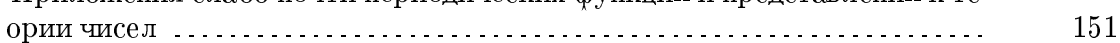

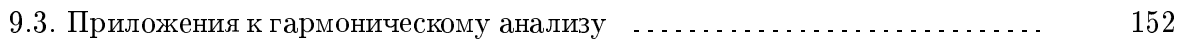

9.4. Приложения к устойчивости представлений абелевых полугрупп и к спек-

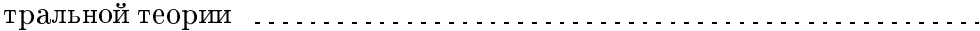

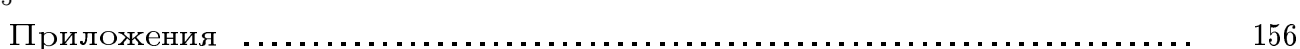

Приложение 1. Полнота по фон Нейману и свойство Крейна ... . . . . . . . . 156

Приложение 2. Интегрирование операторных функций ............... 157

Список литературы . . . . . . . . . . . . 159

В статье Евгении Александровны Бредихиной "Почти периодическая функция" в четвертом томе "Математической энциклопедии" (вьшедшем в 1984 г., почти через 10 лет после смерти автора) почти периодической функцией назьвается “функция, которая может быть представлена обобщенным рядом Фурье”. Конечно, в этой дефиниции можно увидеть и предпочтение определения Бохнера [41] определению Бора [46], но главное - решительное включение предмета в круг явлений, связанных с унитарными представлениями групп и теорией апроксимации. С этой точки зрения уже теория неба по Евдоксу вьплядит предшественницей частных сумм рядов Фурье (ведь именно Евдоксу принадлежит первое инфинитное определение равенства отношений отрезков, во многом похожее на сечение Дедекинда, так что есть на свете веши более тонкие, чем движение планет, которым, по Евдоксу, и трех-четырех гармонических слагаемых довольно). Как и в свое время правильные многогранники (в “Тимее” Платона, см. [197]), так и почти периодические функщии Бора, едва родившись, оказались полезньми в физике (при формировании условия рекуррентности в замкнутых термодинамических системах у Эренфеста, см. [180]). Тем самым темп развития

Исследование поддержано грантом РФФИ 02-01-00574 и программой поддержки ведущих научных школ (проект НШ-619.2003.1). 
понятий в этой области невысок, да и не все можно гармонизировать. Но, хотя и в новейшее время основное понятие почти периодической функции формировалось медленно (история почти периодических функций отражена во многих книгах и статьях, см., например, [43], [140], [141], [149], [189], [219]), успех этого понятия грандиозенпубликации, посвященные почти периодическим объектам (прежде всего - функциям и представлениям груп и полугрупп), а также явно использующие сведения о таких объектах, составляют многие тысячи статей и книг.

Одной из наиболее часто цитируемых в этом круге является книга Б. М. Левитана и В. В. Жикова "Почти периодические функции и дифференциальные уравнения", вьшедшая в 1978 г. [141]. Ее роль была в свое время чрезвычайно велика, поскольку книга подвела итоги большого периода развития и способствовала уяснению связей между почти периодическими функциями и скалярно почти периодическими функциями со значениями в банаховом пространстве с общими вопросами линейного анализа. С тех пор прошло более четверти века, и теория почти периодических функщий и представлений развивалась в это время не менее успешно и применялась в решении многообразных задач (прежде всего - в теории обькновенных дифференциальных уравнений, в том числе - в абстрактных пространствах, и дифференциальных уравнений с частными производньми; оба эти вида приложений заслуживают отдельного обсуждения, но применения почти периодических функций гораздо шире, - см., например, обзоры [99], [130], [196], [205]). Описание материала, добытого в каждом из этих направлений, было бы весьма полезным (ведь в [130] авторам пришлось приводить список нужных им фактов теории почти периодических функций - не нашлось удобных ссылок!), но и гораздо более ограниченная задача - изложить современное состояние запаса основных инструментов собственно теории почти периодических функций - требует масштабов толстой книги, а не журнальной статьи, и поэтому тема обзора требует жесткого ограничения.

Одним из мошных направлений современной теории почти периодических функщий является изучение почти периодических функций со значениями в векторных пространствах, не обязательно нормированных, но, как правило, локально вьпуклых. Как стало ясно уже в 50-е годы, эти вопросы связаны с задачами теории представлений (не только унитарных и не только конечномерных). Это направление имеет многообразные приложения, как традиционные - к теории дифференциальных уравнений, так и сравнительно новые - к теории аналитических или обобщенных аналитических функций и к теории чисел. Чтобы не дать приложениям поглотить бо́льшую часть текста, мы будем по возможности избегать ссылок на работы, посвященные приложениям почти периодических функций к дифференциальным уравнениям и аналитическим функциям, и сосредоточимся прежде всего на классических вопросах собственно теории применительно к почти периодическим функциям на группах с векторными значениями, включая характеризации различных классов почти периодических функций (в том числе - критерии почти периодичности), сушествование среднего значения, условия счетности спектра, теоремы об интеграле и о разностях и связь между сильной, скалярной и слабой почти периодичностью. Следует отметить, что, хотя решение этих задач в частном случае функций со значениями в банаховых пространствах известно специалистам, но и оно недоступно большинству читателей из-за редкости источников и неполной ясности текстов. Кроме того, оказьвается, что для рас- 
пространения известных результатов о почти периодических функциях и слабо почти периодических функциях со значениями в банаховых пространствах и о соответствующих представлениях в банаховых пространствах на случай функций со значениями в локально вьпуклых пространствах и соответствуюших представлений сушественно $^{1}$, чтобы пространство было бочечным (или по крайней мере пространством Макки [203]) и удовлетворяло либо условию полноты, введенному фон Нейманом в 1935 г. либо, в задачах о слабо почти периодических функциях, условию полноты, явным образом введенному Р. Муром в 1968 г. и по существу связанному с известной теоремой Крейна о слабой компактности замкнутой оболочки слабо компактного множества в банаховом пространстве (оба эти условия, вообе говоря, менее ограничительны, чем часто используемое условие квазиполноты), в то время как за пределами этих условий имеют место любопытные патологии, что можно показать на примерах весьма общего характера. В качестве вспомогательных средств изложения используется, в частности, теория де Лю-Гликсберга компактных полутопологических полугрупп и их представлений (основные факты этой теории напоминаются, поскольку изложения этого материала на русском языке недостаточно полны и недостаточно надежны) и связь между условиями слабой и сильной непрерьвности представлений некоторых топологических групп и полугрупп. Эта часть изложения во многом основана на работах Арендта, Басита, Баскакова, Гюнщлера, Чжана, Жикова, Кадеца, Левитана, де Лю и Любича и в некоторой степени унифищирует собранньй в этих работах материал. С другой стороны, обзор содержит информащию о роли теории почти периодических и особенно слабо почти периодических функций в теории представлений груш и полугрупп и гармоническом анализе на этих объектах, включая свойства простейших компактификаций групп и полугрупп. Здесь использованы достижения Бордбара, Гликсберга, Кромбэ, Э. Лау, Мегрелишвили, Милна, Пима, Чоу и ряда других авторов. Теоремы, помеченные буквами, - цитаты, а нумерованные теоремы являются новыми утверждениями.

Изложение ведется следующим образом. В 11 изложены основные определения, список которых в дальнейшем придется постоянно пополнять, и приведены необходимые сведения из теории локально выпуклых пространств. В $\S 2$ напоминаются важнейшие факты общей теории компактных полутопологических полугрупп и их представлений. В 33 они используются для построения аналога разложения де Лю-Гликсберга для слабо почти периодических представлений в бочечных пространствах со свойством Крейна. В $\S 4$ полученное разложение применяется в установлении условий почти периодичности одновременно слабо и скалярно почти периодических представлений. В $\S 5$ указаны приложения полученных результатов к другим фундаментальным вопросам теории почти периодических функций с векторными значениями, включая условия счетности спектра, теоремы об интеграле и о разностях. В $\S 6$ разобраны некоторые примеры. В $\S 7$ приведены результаты, связанные с классическими задачами теории почти периодических функций, а в $\S 8$ - обзор других направлений исследования почти периодических функций и почти периодических представлений груп.

\footnotetext{
${ }^{1}$ Но не необходимо. Используя технику $l^{\infty}$-бочечных пространств и некоторые другие ослабления условия бочечности, можно существенно расширить запас примеров, в которых справедлива теорема разложения и ее следствия. Однако здесь мы не стремимся к достижению наилучших возможных результатов, ограничиваясь тем, что может быть получено с помощью общеизвестных приемов. Как мы надеемся, это позволит сосредоточить внимание читателя не на трудностях анализа, а на взаимодействии основных идей.
} 
Некоторые смежные задачи и приложения обсуждаются в $\S$. Заключительные замечания высказаны в $\S 10$. В приложениях обсуждаются условия полноты локально вьпуклых пространств, используемые в работе, и напоминаются свойства "слабого интеграла" от векторных и операторных функций в локально выпуклых пространстBax.

Автор считает своим приятньм долгом поблагодарить Э. Гранирера, Г. Гюнцлера и Э. Т.-М. Лау за любезно предоставленные оттиски их работ, П. Милнса за электронную версию одной из его публикаций и доктора А. Хотцеля за помошь в подготовке материалов статьи.

\section{$\S$ 1. Введение. Основные определения. Обозначения и терминология}

По всем вопросам, касающимся топологических векторных пространств, мы отсылаем читателя к [195], если понятие, определение или факт не снабжены точной ссылкой. Как обычно, условимся, что символ * означает переход к сопряженному пространству или оператору, $\sigma(E, F)$ означает слабую топологию на локально выпуклом пространстве $E$, порожденную двойственностью $\{E, F\}$ с локально выпуклым пространством $F$, а $\tau(E, F)$ означает топологию Макки на локально вьпуклом пространстве $E$, порожденную двойственностью $\{E, F\}$. Мы также используем ссылки на [76], которые всегда явно указаны. Для ссылок по теории представлений групп мы используем книги [147] и [171], по теории инвариантных средних - [97] и [177], по общей топологии - [80] и по теории нормированных линейных пространств - [66].

Начнем с определения полутопологической полугруппы.

ОПРЕДЕЛЕНИЕ 1. Полутопологической полугруппой называется полугруппа с единичньм элементом, снабженная хаусдорфовой топологией, в которой умножение является раздельно непрерывным. Пусть $S$ - полутопологическая полугруппа. Напомним, что подполугруппой в $S$ называется такое непустое подмножество $M \subset S$, что $M M \subset M$, левым (правым $)$ идеалом в $S$ называется такое подмножество $M \subset S$, что $S M \subset M(M S \subset M)$, и $M$ называется двусторонним идеалом в $S$, если $M$ является одновременно левьм и правым идеалом в $S$. Пересечение $K(S)$ всех двусторонних идеалов в $S$ назьвается ядром $S$. Элемент $e \in S$ назьвается идемпотентом в $S$, если $е e=e$. Если $M \subset S$, обозначим через $\mathscr{E}(M)$ множество всех идемпотентов в $S$, принадлежаших $M$. Для множества всех минимальных левых (правых) идеалов в $S$ мы используем символ $\mathscr{L}(S)(\mathscr{R}(S))$. Компактной полутопологической полугруппой мы называем полугруппу с единицей и с раздельно непрерьвным умножением, топологическое пространство которой компактно.

Пусть $S$ - полутопологическая полугруппа, $E$ - локально вьпуклое пространство, а $\pi$ - представление полугруппы $S$ в $E$. Если явно не оговорено противное, в дальнейшем всегда предполагается, что представление $\pi$ слабо непрерьвно. Обозначим символом $\mathrm{CB}(S)$ банахово пространство непрерьвных ограниченных числовых функций на $S$ в равномерной топологии.

ОПРЕДЕЛЕНИЕ 2. КаК обычно, мы будем говорить, что вектор $x \in E$ no чmu nepuодичен (по Бохнеру) относительно представления $\pi$, если замыкание соответствующей орбиты $\mathscr{O}(x)=\{\pi(s) x \mid s \in S\}$ компактно в $E$ (в исходной топологии пространства $E$ ). Далее, вектор $x \in E$ назьвается слабо почти периодическим (по Эберлейну) относительно представления $\pi$, если слабое замыкание орбиты $\mathscr{O}(x), x \in E$, является 
слабо компактным множеством в $E$. Представление $\pi$ назьвается почти периодическим (слабо почти периодическим), если все векторы $x \in E$ почти периодичны (слабо почти периодичны). Представление $\pi$ назьвается скалярно почти периодическим ${ }^{2}$, если каждый "матричньй элемент" $g \mapsto F(f(g)), g \in G$, где $F \in E^{*}$, является числовой функцией, почти периодической в смысле Бохнера. Представление $\pi$ называется скалярно слабо почти периодическим, если каждый "матричный элемент" $g \mapsto F(f(g)), g \in G$, где $F \in E^{*}$, является числовой функцией, слабо почти периодической в смысле Эберлейна.

Пусть $\lambda(\rho)$ - левое (правое) регулярное антипредставление (представление) группы $G$ в пространстве $\mathrm{CB}(G)$ ограниченных непрерьвных функций на $G$, определенное левыми (правыми) сдвигами на $\mathrm{CB}(G)$, т.е. формулами

$$
\begin{aligned}
& \lambda(t) f(s)=f(t s), \quad s, t \in S, \quad f \in \mathrm{CB}(G), \\
& \rho(t) f(s)=f(s t), \quad s, t \in G, \quad f \in \mathrm{CB}(G) .
\end{aligned}
$$

Числовая функция $f \in \mathrm{CB}(S)$ называется почти периодической (по Бохнеру, ср. [141]), если $f$ является слабо почти периодическим вектором относительно левого регулярного антипредставления (или, что равносильно, правого регулярного представления [97]) в пространстве $\mathrm{CB}(S)$. Функция $f \in \mathrm{CB}(S)$ называется слабо почти периодической (по Эберлейну, ср. [74]) если $f$ является слабо почти периодическим вектором относительно левого регулярного антипредставления (или, что равносильно, правого регулярного представления [97]) в пространстве $\mathrm{CB}(S)$.

Множество $W A P(S)$ всех слабо почти периодических функций, принадлежащих пространству $\mathrm{CB}(S)$, является замкнутой $S$-инвариантной равномерной самосопряженной подалгеброй алгебры $\mathrm{CB}(S)$, содержащей единичньй элемент. Спектр $S_{w}$ (пространство максимальных идеалов) коммутативной банаховой алгебры $W A P(S)$ является компактным топологическим пространством, снабженным естественньм умножением, превращающим $S_{w}$ в компактную полутопологическую полугруппу (см. определение 3 в $\S 2$ ниже). Эта полугруппа играет ключевую роль в доказательстве следуюшего аналога знаменитой теоремы Гельфанда-Райкова (см., например, [147]): топологическая группа имеет достаточно много слабо непрерывных представлений изометрическими операторами в рефлексивных банаховых пространствах тогда и только тогда, когда эта топологическая група допускает непрерьвное вложение в компактную полутопологическую полугрупу [198]. (Доказательство этого факта существенно использует результаты статьи [227].) Основные сведения о компактных полутопологических полугруппах и их обобщениях можно найти в [35]-[37], [51], [189]. Полезным новым обшим руководством по почти периодическим функциям и слабо почти периодическим функциям со значениями в банаховом пространстве является диссертация Чжана [230].

В следующем определении мы вводим основные классы почти периодических функций со значениями в локально вьпуклом пространстве. Это определение является обязьваюшим, поскольку имеет много вариантов. Если идея аппроксимации выражена условием той или иной компактности, а идея ряда Фурье - групповой структурой и топологией на групе, определяющей список непрерывных представлений,

\footnotetext{
${ }^{2}$ Во многих источниках, включая [2] и [141], эти представления называются слабо почти периодическими. Мы придерживаемся терминологии, сложившейся в два последних десятилетия.
} 
то варьировать в определении можно и топологию в пространстве непрерывных векторнозначных функций, и само условие непрерьвности этих функций. В статье фон Неймана и Бохнера [43] (одной из первых по теории почти периодических функций с векторньми значениями и развивавшей идеи статьи [46], связанной со скалярнозначными функциями) нет никакого условия непрерывности функций (требуется только ограниченность образа функции как подмножества локально вьпуклого пространства значений), и единственньм дополнительным требованием является условие предкомпактности семейства сдвигов рассматриваемой функции относительно естественной равномерности, определяемой топологией пространства-образа. Этот класс функций слишком удален от почти периодических функций Бора, которые заведомо непрерьвны. Поэтому в основном определении мы сохраним условие непрерьвности, но, чтобы несколько расширить класс, от элементов которого обычно требуется сильная непрерьвность (в банаховом случае так поступают многие авторы, см., например, [6], [10], [15], [27], [33], [35], [36], [51], [56], [90], [108], [123], [157], [185], [187]), мы рассмотрим класс слабо непрерывных функций (в банаховом случае так поступили де Лю и Гликсберг [67] и Чжан в [230]), более широкий, чем боровский (по крайней мере формально), с естественным дополнительным условием компактности, и введем его родственные классы. Это определение согласовано с определением 1 и было принято в [120], [202], [203] для функций со значениями в локально вьпуклых пространствах.

ОПРеДЕЛЕНИЕ $3 .^{3}$ Пусть $E$ - локально выпуклое пространство, $S$ - полутопологическая полугруппа (см. определение 1$), \mathscr{U}$ - база окрестностей нуля в $E, U$ - окрестность нуля в $E$, а $U^{\prime}$ - семейство функций $g: S \rightarrow E$, принимающих значения в $U$. Пусть $\mathscr{U}^{\prime}=\left\{U^{\prime} \mid U \in \mathscr{U}\right\}$. Пусть В $(S, E)$ - локально выпуклое пространство, образованное функциями $g: S \rightarrow E$, множества значений которых ограничены в пространстве $E$, и снабженное базой окрестностей нуля $\mathscr{U}^{\prime}$ [43], [220]. Обозначим символом $\mathrm{CB}(S, E)$ локально вьпуклое пространство непрерьвных ограниченных $E$-значных функций на $S$ в равномерной топологии, т.е. в топологии, индуцированной на подпространстве $\mathrm{CB}(S, E)$ указанной вьше топологией на пространстве $\mathrm{B}(S, E)$.

Функция $f: S \rightarrow E$ называется почти периодической в смысле фон Неймана, если ее левая и правая орбиты (т.е. семейства левых и правых сдвигов функции $f$ ) вполне ограничены в $\mathrm{B}(S, E)$ в только что определенной топологии. Если в этом определении заменить полную ограниченность в $E$ слабой полной ограниченностью в $\mathrm{B}(S, E)$, мы получим определение слабо почти периодической функиии в смысле фон Неймана.

Пусть $E$ - локально вьпуклое пространство, $S$ - полутопологическая полугруппа, а $W C B(S, E)$ - пространство ограниченных функций на $S$ со значениями в $E$, которые оказьваются непрерьвньми, если пространство $E$ снабжено слабой топологией $\sigma\left(E, E^{*}\right)$. Пусть пространство $W C B(S, E)$ снабжено некоторой локально выпуклой топологией $\beta$, согласованной с топологией простой слабой сходимости на $S$. Функция $f: S \rightarrow E$ назьвается $\beta$-почти периодической (или $\beta$-почти периодической в смысле Бохнера), если семейство левых (или правых) сдвигов функции $f$ имеет компактное замькание по отношению к топологии $\beta$ в пространстве $W C B(S, E)$. Если семейства

\footnotetext{
${ }^{3}$ Семейство естественных топологий в пространстве в том или ином смысле непрерывных функций со значениями в локально выпуклом пространстве намного богаче скудного банахова рациона, и каждьй выбор приводит к своему классу почти периодических функций. Читатель может найти начало списка полезных топологий такого рода в [129].
} 
левых и правых сдвигов функции $f$ имеют слабо компактные замькания, то функция $f$ назьвается $\beta$-слабо почти периодической функиией в смысле Эберлейна.

Пусть $E$ - локально вьпуклое пространство, $S$ - полутопологическая полугруппа. Функция $f: G \rightarrow E$ называется скалярно почти периодической в смысле фон Неймана, если каждый “матричный элемент" $g \mapsto F(f(g)), g \in G$, где $F \in E^{*}$, является числовой функцией, почти периодической в смысле фон Неймана. Функция $f: G \rightarrow E$ называется скалярно слабо почти периодической, если каждый “матричный элемент" $g \mapsto F(f(g)), g \in G$, где $F \in E^{*}$, является числовой функцией, слабо почти периодической в смысле Эберлейна.

Даже для функций со значениями в банаховых пространствах различие между условиями предкомпактности в определении фон Неймана и относительной компактности в определениях Бохнера и Эберлейна сушественно (см. [157]).

Очевидно, что любая почти периодическая функция является слабо почти периодической. Кроме того (ср. [35]), нетрудно видеть, что все перечисленные пространства инвариантны относительно сдвигов и имеют свойство Крейна, если $E$ имеет свойство Крейна.

Каждое из перечисленных вьше условий, относяшееся к правым сдвигам, равносильно соответствуюшему условию относительно левых сдвигов. Вот схема (для простоты - для числовых функций). Пусть $S$ - топологическая полугрупша, $\mathrm{CB}(S)$ - банахово пространство ограниченных непрерывных комплексных функций на $S$, a $f$ - функшия, принадлежашая банахову пространству $W_{R}(S)$ всех функций из $\mathrm{CB}(S)$, правая орбита которых

$$
O_{R}(f)=\left\{R_{s} f \mid s \in S\right\}, \quad R_{s} f(t)=f(t s), \quad t, s \in S,
$$

относительно слабо компактна в $\mathrm{CB}(S)$. Пусть $S^{w}$ - замыкание полугруппы $\left\{R_{s} \mid\right.$ $s \in S\}$ в слабой операторной топологии. Пусть $R: S \rightarrow S^{w}$ - непрерывный гомоморфизм, определенный формулой $R(s)=R_{s}, s \in S$, и пусть $\widetilde{R}: \mathrm{CB}\left(S^{w}\right) \rightarrow \mathrm{CB}(S)$ - соответствуюшее индуцированное отображение. Для любой функции $f \in W_{R}(S)$ обозначим ее прообраз через $F$, т.е. положим $F=\widetilde{R}^{-1}(f) \in \mathrm{CB}\left(S^{w}\right)$. Очевидно, что любой левьй сдвиг ее прообраза (функция вида $L_{R_{s}} F$ ) содержится в $\mathrm{CB}\left(S^{w}\right)$, где использовано стандартное обозначение $L_{s} f(t)=f(s t)$. Тогда

$$
\begin{aligned}
\widetilde{R}\left(L_{R_{s}} F\right)(t) & =L_{R_{s}} F(R(t))=L_{R_{s}} F\left(R_{t}\right)=F\left(R_{s} R_{t}\right) \\
& =F(R(s t))=\widetilde{R}(F)(s t)=f(s t)=\left(L_{s} f\right)(t) .
\end{aligned}
$$

Следовательно, семейство $W_{R}(S)$ инвариантно относительно левых сдвигов $\left(L_{s} f=\right.$ $\left.\widetilde{R}\left(L_{R_{s}} F\right)\right)$, и левая орбита функции $F$ содержится в левой орбите $f$. Разумеется, это рассуждение справедливо для слабо почти периодических полугруш операторов в локально выпуклых пространствах (ср. лемму 1 и теорему 2 ниже).

Понятие слабо почти периодической функщии (на абелевой локально компактной группе) ввел Эберлейн [74]. Он также показал [75], что любая слабо почти периодическая функция на абелевой локально компактной группе может быть представлена в виде суммы почти периодической функции и “диссипативной” слабо почти периодической функции. Это соотношение было затем распространено (в [51], [115], [116]) на случай полугруппы и в [67] на случай топологической полугрупшы $S$, допускающей инвариантное среднее на пространстве ограниченных непрерьвных функций на $S$. 
Этот результат приобрел универсальньй характер, когда Рылль-Нардзевский доказал свою знаменитую теорему о неподвижной точке [191] и немедленно применил ее в доказательстве существования инвариантного среднего на пространстве слабо почти периодических функций на любой топологической групше.

В заключение параграфа следует сказать несколько слов о "почти периодах", т.е. об определении почти периодических функций “по Бору”. Пусть $G$ - топологическая группа и $R_{s}, s \in G,-$ операшия правого сдвига $\left(\left(R_{s} f\right)(t)=f(s t), t \in G, f\right.$ - комплексная функция на $G$ ). Непрерьвная функция $f$ на $G$ называется правой почти периодической по Бору, если для любого $\varepsilon>0$ существует такое компактноемножество $K_{\varepsilon} \subset G$, что $A_{\varepsilon} K_{\varepsilon}=G$, где $A_{\varepsilon}=\left\{s \in G|| R_{s} f(t)-f(t) \mid<\varepsilon\right.$ для всех $\left.t \in G\right\}$. Левые почти периодические функции определяются аналогично. Оказывается, классы правых и левых почти периодических функций по Бору не обязаны совпадать между собой [223] или с пространством почти периодических функций по Бохнеру [164] и не обязаны быть векторньми пространствами [160] даже в случае, если $G$ - локально компактная группа.

Ниже мы обсудим взаимоотношения между условиями слабой почти периодичности и почти периодичности слабо непрерьвных представлений топологических групп в локально вьпуклом пространстве. Соответствуюшие явления связаны с фактами известной теории компактных полутопологических полугруп, компактных операторных групп и полугрупп и с соотношениями между условиями слабой и сильной непрерьвности представлений групп в локально выпуклых пространствах и между условиями раздельной непрерьвности (непрерывности по каждому переменному в отдельности) и совместной непрерьвности (непрерьвности по совокупности переменных) для действий групп и полугрупп и умножений в этих алгебраических объектах. По этой причине мы напомним здесь некоторые основные результаты, относяшиеся к этому кругу явлений.

\section{$\S 2$ Основные факты теории де Лю-Гликсберга}

Приведем нужные нам в дальнейшем результаты о полутопологических полугруппах в виде следующих теорем A-E.

ТЕОРема А (Эллис, [77]). Компактная полутопологическая полугруппа, которая алгебраически является группой, есть компактная топологическая группа (т.е. умножсение является совместно непрерывным и операция обращения тоже непрерывна). ${ }^{4}$

ТеОРема В [67; 2.2-2.9]. Пусть $S$ - компактная полутопологическая полуzpynna.

(1) Справедливы следующие утверждения:

\footnotetext{
${ }^{4}$ Лоусон [139; следствие 6.3] доказал более сильное утверждение: любая подгруппа компактной полутопологической полугруппы является топологической группой. В дальнейшем условия непрерывности для действий полугрупп и условия того, что раздельно непрерьвное умножение в полугруппе имеет достаточно много точек совместной непрерывности или даже определяет топологическую полугруппу, рассматривались с различных точек зрения, в частности, в [64], [67], [78], [93], [107], [110], [139], [153], [174]; соответствующие резултаты существенно уточняют теоремы Эллиса и Лоусона, но эти более сильные утверждения нам не понадобятся.
} 
(1a) множество $K(S)$ непусто $u$

$$
\begin{aligned}
& \mathscr{L}(S)=\{S e \mid e \in \mathscr{E}(K(S))\}, \\
& \mathscr{R}(S)=\{e S \mid e \in \mathscr{E}(K(S))\}, \\
& K(S)=\bigcup_{I \in \mathscr{L}(S)} I=\bigcup_{I \in \mathscr{R}(S)} I ;
\end{aligned}
$$

(1b) если $J_{1}, J_{2} \in \mathscr{L}(S)\left(J_{1}, J_{2} \in \mathscr{R}(S)\right)$ и пересечение $J_{1} \cap J_{2}$ непусто, $m o J_{1}=J_{2}$

(1c) если $J \in \mathscr{L}(S)(J \in \mathscr{R}(S))$, то $J s=J(s J=J)$ для любьх $s \in J$;

(1d) если $J_{1} \in \mathscr{L}(S)$ и $J_{2} \in \mathscr{R}(S)$, то пересечение $J_{1} \cap J_{2}$ непусто $и$ содержит единственный идемпотент, который мы обозначим через е; этот идемпотент удовлетворяет условию $J_{1} \cap J_{2}=e S e, u J_{1} \cap J_{2}$ компактная топологическая группа с единичным әлементом е.

(2) Следующие условия әквивалентнь:

(2а) пространство $\mathrm{C}(S)$ всех непрерывных функций на $S$ допускает (двусторонне) инвариантное среднее (т.е. существует такой непрерывный линейный функционал $m$ на $\mathrm{C}(S)$, что $\|m\|=1, m(\mathbf{1})=1$, где $\mathbf{1}$ обозначает функцию, тождественно равную $1, u$

$$
m(\rho(s) f)=m(\lambda(s) f)=m(f) \quad \text { для любъх } \quad s \in S, \quad f \in \mathrm{C}(S),
$$

или, что равносильно, для всех $s$ из некоторого подмножества $M \subset S$, плотного в $S$ );

(2b) $S$ имеет единственный минимальный левьй идеал и единственный минимальный правый идеал, эти идеаль совпадают, и каждый из них совпадает с $K(S)$;

(2c) $K(S)$ - компактная топологическая группа, и инвариантное среднее на $S$ определено интегрированием по мере Хаара на $K(S)$.

Приведем еше один результат статьи [67], связанньй со средними на пространстве непрерьвных функций на компактной полутопологической полугруппе.

Теорема С $[67 ; 2.7$ и 2.10$]$.

(1) Пусть $S$ - компактная полутопологическая полугруппа, $f$ - функция из $\mathrm{C}(S)$, и пусть $\mathscr{O}(f)=\{\rho(s) f \mid s \in S\}$. Тогда множество $\mathscr{O}(f)$ слабо компактно в $\mathrm{C}(S)$ и отображение $s \rightarrow \rho(s) f, s \in S$, слабо непрерывно. Если $\mathscr{O}(f)$ сильно относительно компактно, то отображение $s \rightarrow \rho(s) f$, $s \in S$, сильно непрерывно.

(2) Пусть $S$ - полутопологическая полугруппа, $S^{\prime}$ - компактная полутопологическая полугруппа, а $\varphi: S \rightarrow S^{\prime}$ - непрерывный гомоморфизм, причем его образ $\varphi(S)$ плотен в $S^{\prime}$. Пусть $\widetilde{\varphi}: \mathrm{C}\left(S^{\prime}\right) \rightarrow \mathrm{CB}(S)$ - отображсение, заданное формулой $f \mapsto f \circ \varphi$ для любой функиии $f \in \mathrm{C}\left(S^{\prime}\right)$. В этом случае пространство $\mathrm{C}\left(S^{\prime}\right)$ допускает двусторонне инвариантное среднее тогда и только тогда, когда его образ $\widetilde{\varphi}\left(\mathrm{C}\left(S^{\prime}\right)\right)$ допускает двусторонне инвариантное среднее.

Кроме того, напомним основные свойства слабо почти периодических функций на полутопологической полугруппе. 
Teopema D $[67 ; 5.1-5.11]$.

(1) Если $S$ - компактная полутопологическая полугруппа, то $W A P(S)=$ $\mathrm{C}(S)$. Кроме того, если умножение в $S$ непрерывно по совокупности переменных ("совместно непрерывно"), то $A P(S)=\mathrm{C}(S)$.

(2) Пусть $S$ и $S^{\prime}$ - полутопологические полугруппы, $\varphi: S \rightarrow S^{\prime}-$ непрерывный гомоморфизм, а $\widehat{\varphi}-$ отображение, заданное формулой $\widehat{\varphi} f=f \circ \varphi$ для любой функиии $f \in \mathrm{CB}\left(S^{\prime}\right)$. Тогда $\widehat{\varphi}\left(W A P\left(S^{\prime}\right)\right) \subset W A P(S)$ и $\widehat{\varphi}\left(A P\left(S^{\prime}\right)\right) \subset$ $A P(S)$. Если $S^{\prime}$ компактна, то $\widehat{\varphi}\left(\mathrm{C}\left(S^{\prime}\right)\right) \subset W A P(S)$, а если к тому же и умножсение в $S^{\prime}$ непрерывно по совокупности переменных, то $\widehat{\varphi}\left(\mathrm{C}\left(S^{\prime}\right)\right) \subset$ $A P(S)$.

(3) Пусть $S$ - полутопологическая полугруппа и $\mathrm{CB}(S)$ - пространство непрерывных ограниченных комплекснозначных функций на $S$. Сохраним символ $\rho(s)$ для ограничения оператора $\rho(s), s \in S$, на подпространство WAP $(S)$ пространства $\mathrm{CB}(S)$. Эти ограничения образуют слабо почти периодическую полугруппу операторов в банаховом пространстве $W A P(S)$. Обозначим через $S^{w}$ замыкание әтой операторной полугруппь в слабой операторной топологии; тогда $S^{w}$ - компактная полутопологическая полугруппа (так назыввемая слабо почти периодическая компактификация полугруппы $S$ ). Гомоморфизм $S \rightarrow S^{w}$, переводящий $s \in S$ в $\rho(s)$, непрерывен. Соответствующее отображение, определенное в п. (2) этой теоремь, является изоморфизмом алгебри $\mathrm{C}\left(S^{w}\right)$ на алгебру $W A P(S)$.

(4) Если $S$ - компактная полутопологическая полугруппа, то гомоморфизм $S \rightarrow S^{w}$, переводящий $s \in S$ в $\rho(s)$ (см. утверждение (3)), является топологическим изоморфизмом топологической полугруппы $S$ на $S^{w}$.

(5) Если $S$ и $S^{\prime}$ - полутопологические полугруппы $и \varphi: S \rightarrow S^{\prime}$ - непрерывный гомоморфизм, то существует такой непрерывный гомоморфизм $\varphi^{w}: S^{w} \rightarrow S^{\prime w}$, чтоо $\varphi^{w}(\rho(s))=\rho(\varphi(s))$ для любых $s \in S$.

(6) Пусть $S$ - топологическая группа, $S^{\prime}$ - компактная полутопологическая полугруппа, $\varphi: S \rightarrow S^{\prime}$ - непрерывныи гомоморфизм, и пусть множество $\varphi(S)$ плотно в $S^{\prime}$. Если отображение, определенное формулой $\widehat{\varphi} f=f \circ \varphi$ для любых $f \in \mathrm{C}\left(S^{\prime}\right)$, переводит $\mathrm{C}\left(S^{\prime}\right)$ на $W A P(S)$, то существует такой топологический изоморфизм $\psi: S^{w} \rightarrow S^{\prime}$ на $S^{\prime}$, что ${ }^{5} \psi(\rho(s))=\varphi(s)$ для любых $s \in S$.

(7) Пусть $S$ - полутопологическая полугруппа. Воспользуемся понятиями, введенными ниже в определении 4 в 3 . Следующие три условия эквивалентны:

(a) $W A P(S)$ допускает инвариантное среднее,

(b) ядро полугруппь $S^{w}$ - компактная топологическая группа,

(c) $W A P(S)_{r}$ совпадает с пространством $A P(S), W A P(S)_{v}$ является замкнутыцм подпространством в $W A P(S)$, инвариантным относительно сдвигов, и WAP(S) - прямая сумма $A P(S)$ и $W A P(S)_{v}$.

\footnotetext{
${ }^{5}$ Таким образом, $S^{w}$ - единственная (с точностью до топологического изоморфизма) компактная полугруппа, в которую полугруппу $S$ можно непрерывно вложить как плотную подполугруппу так, что каждая функция из $W A P(S)$ допускает непрерьвное продолжение на $S^{w}$. Мь будем обозначать полугруппу $S^{w}$ символом $G^{\mathscr{W} \mathscr{A} \mathscr{P}}$.
} 
Если эти әквивалентные условия (а)-(с) выполнены, то инвариантное среднее $m$ на $W A P(S)$ единственно, и оно определяется интегрированием функиии из $\mathrm{C}\left(S^{w}\right)$ по мере Хаара на компактной группе $K\left(S^{w}\right)$ после применения изоморфизма между $\mathrm{C}\left(S^{w}\right)$ u WAP(S), указанного в утверждении (3). Кроме того, $W A P(S)_{v}$ совпадает с множеством $\left\{f \mid f \in W A P(S), m\left(|f|^{2}\right)=0\right\}$.

Напомним теперь следствие из теоремы Рылль-Нардзевского о неподвижной точке.

ТеОрема Е [191]. Для любой топологической группь G существует единственное двусторонне инвариантное среднее на алгебре $W A P(G)$ слабо почти периодических комплекснозначных функций на $G$.

Таким образом, все утверждения п. (7) теоремы D выполняются, если $S$ - топологическая группа.

Как уже было сказано вьше, еше одной общей темой, связанной с условиями непрерьвности почти периодических функций, является связь между условиями сильной и слабой непрерывности представлений групп и полугруп, которая исследовалась многими авторами. Условия непрерьвности борелевских (а позже и измеримых) гомоморфизмов и сильной непрерывности слабо непрерывных гомоморфизмов, а также непрерывности гомоморфизмов в терминах вариации в точке рассматривались, начиная с [9], в большом числе работ, в том числе - де Лю и Гликсбергом [68], Р. Муром [168], Лоусоном [139], Ханселем и Троалликом [107], Хельмером [110], Мегрелишвили [153], Баситом и Прайдом [20], Нибом и Пикреллом [174]. В частности, в [68] де Лю и Гликсберг доказали, что слабо непрерьвное представление локально компактной групшы в любом банаховом пространстве автоматически сильно непрерьвно, и приписали это доказательство Миркилу. Это доказательство слишком кратко, а подробное доказательство приведено Джонсоном в [117]. ${ }^{6}$ Обзор новых результатов в этом направлении дан в [153]. Часть этих результатов связывает сильную непрерьвность слабо непрерьвных представлений груп с топологическими свойствами группы (локальной компактностью [168], топологической полнотой (см. обзорную часть в [202])), а другие утверждения связаны с условиями геометрического характера, накладьваемьми на пространство представления или на орбиты представления (например, свойство Радона-Никодима [173], [206], [207]), свойство Асплунда [207], [226] ${ }^{7}$, свойство точек непрерьвности, фрагментируемость в смысле статей [153], [173], бочечность локально вьпуклого пространства представления ([168], см. также обзорную часть работы [153]). Некоторые новые результаты о связи между условиями слабой и сильной непрерывности представлений групп в банаховых пространствах приведены в [200], [202].

Здесь уместно отметить следующую постановку задачи, обобщающую приведенную вьше и изученную в [119]. Пусть $S$ - полутопологическая полугруппа, и пусть

\footnotetext{
${ }^{6}$ В книге Любича [147] автор ошибочно использет характеристические функции окрестностей вместо непрерывных функций с компактным носителем, использование которых сделало бы эту часть доказательства правильной, но осталось бы не доказанным, что полученный интеграл является элементом исходного банахова пространства, а не только его второго сопряженного.

${ }^{7}$ Свойства Радона-Никодима, Данфорда-Петтиса и Асплунда встретятся нам ниже в $\S 8$ при обсуждении геометрических свойств групповых алгебр.
} 
отображение $\phi: S \rightarrow S^{\prime}$ гомеоморфно отображает $S$ на плотное подмножество компактной правотопологической полугрупш $S^{\prime}$ (правая топологичность означает, что умножение непрерьвно только по левому переменному при фиксированном правом сомножителе) и, кроме того, левые сдвиги $S^{\prime} \rightarrow S^{\prime}, x \mapsto \phi(s) x, x \in S^{\prime}$, непрерьвны для любых $s \in S$. Доказано, что если $\pi: S^{\prime} \rightarrow I$ - непрерывная гомоморфная ретракция на правый идеал $I$ в $S^{\prime}$, то $\mathrm{C}\left(S^{\prime}\right)$ допускает разложение в прямую сумму векторных пространств $\mathrm{C}\left(S^{\prime}\right)=\pi^{*}(\mathrm{C}(I)) \oplus \mathrm{CB}\left(S^{\prime}, I\right)$, где $\pi^{*}$ - “гельфандовское преобразование" отображения $\pi$ и $\mathrm{CB}\left(S^{\prime}, I\right)$ - множество всех ограниченных непрерьвных функций на $S^{\prime}$, обрашаюшихся в нуль на $I$; оба эти подпространства инвариантны относительно левых сдвигов на элементы множества $\phi(S)$, а $\mathrm{CB}\left(S^{\prime}, I\right)$ - идеал в алгебре $\mathrm{C}\left(S^{\prime}\right)$. Кроме того, верно и обратное (см. также [167]): если $\psi: S^{\prime} \rightarrow S^{\prime \prime}$ - такой сюръективньй непрерьвньй гомоморфизм, что $\mathrm{C}\left(S^{\prime}\right)$ можно представить в виде прямой суммы $\mathrm{C}\left(S^{\prime}\right)=\psi^{*}\left(\mathrm{C}\left(S^{\prime \prime}\right)\right) \oplus H$, где $\psi^{*}$ - "гельфандовское преобразование" отображения $\psi$ и $H$ - идеал в $\mathrm{CB}\left(S^{\prime}\right)$ (инвариантньй относительно левых сдвигов на элементы $\phi(S))$, то $S^{\prime \prime}$ можно отождествить с таким левым идеалом $I$ в $S^{\prime}$, что $H$ совпадает с пространством всех ограниченных непрерьвных функций на $S^{\prime}$, обращающихся в нуль на $I$. (Этот факт является существенным обобшением разложения слабо почти периодической функции в сумму почти периодической и диссипативной функций. Заметим, что за пределами банаховых модулей над алгеброй числовых почти периодических функций (вне структуры модуля над алгеброй) трудно представить себе язык, на котором возможно векторное обобщение этого результата.)

Перейдем теперь к постановке и решению одной из основных задач этой статьи. ${ }^{8}$

\section{$\S$ 3. Аналог разложения де Лю-Гликсберга для слабо почти периодических представлений в бочечных локально выпуклых пространствах}

Мы собираемся распространить основную конструкцию статьи [67] на случай представлений в локально выпуклом пространстве. С этой целью мы сначала напомним результаты этой статьи, связанные с непрерьвными функциями на компактных полутопологических полугруппах, и обсудим изменения, необходимые при изучении представлений таких полугрупп в локально выпуклых пространствах (так как в [67] рассматривался только случай представлений в банаховых пространствах). Требуемое обобшение результатов де Лю и Гликсберга осушествляется в этом параграфе статьи.

Напомним, что $\mathscr{L}(E)$ - семейство всех непрерывных линейных операторов в локально выпуклом пространстве $E$. Можно рассматривать $\mathscr{L}(E)$ и как полугруппу с единичным элементом, а именно единичньм оператором в $E$. Под nолугрупnой оnepaторов в пространстве $E$ мы понимаем произвольную подполугруппу $S$ в $\mathscr{L}(E)$, содержашую единичньй элемент (оператор). Полугруппа операторов в $E$ назьвается

\footnotetext{
${ }^{8}$ Некоторые новые свойства полутопологических полугрупп приведены в [213]; эти свойства позволяют упростить доказательства ряда известных результатов о компактных полутопологических полугруппах. Троаллик [212], [213] ведет изложение на основе теоремы Намиока [172] о связи между раздельной и совместной непрерьвностью и не пользуется теоремой Рылль-Нардзевского [191] о неподвижной точке. Недавние обобщения теоремы Намиока, полученные в [107] и некоторых других работах, могут оказаться применимыми и в более общей ситуации представлений в локалњно выпуклых пространствах.
} 
слабо почти периодической (почти периодической), если тождественное представление $S$ в пространстве $E$ слабо почти периодично (почти периодично).

Лемма 1. Пусть $S$ - слабо почти периодическая полугруппа операторов в локально выпуклом пространстве E. Тогда замыкание $\bar{S}$ полугруппь $S$ - компактная полутопологическая полугруппа в слабой операторной топологии.

ДоказАТЕльство. Так как умножение в $\mathscr{L}(E)$ раздельно непрерывно в слабой операторной топологии, то замыкание $\bar{S}$ полугрупш $S$ в $\mathscr{L}(E)$ замкнуто относительно умножения. Следовательно, $\bar{S}$ - полутопологическая полугруппа. Докажем, что $\bar{S}$ компактна. Для любого $x \in E$ замыкание $\overline{\mathscr{O}(x)}$ орбиты $\mathscr{O}(x)$ точки $x$ компактно в слабой топологии пространства $E$, причем $T x \in \overline{\mathscr{O}(x)}$ для любых $x \in E$ и $T \in \bar{S}$ по определению замыкания в слабой операторной топологии. Пусть $\chi: \bar{S} \rightarrow \prod_{x \in E} \overline{\mathscr{O}(x)}-$

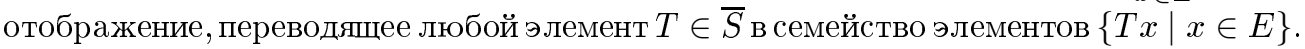
По определению слабой топологии в $E$, это отображение (которое, очевидно, взаимно однозначно) является гомеоморфизмом. Так как произведение $\prod_{x \in E} \overline{\mathscr{O}(x)}$ компактно по теореме Тихонова, то компактность множества $\bar{S}$ будет установлена, если мы докажем, что его образ $\chi(\bar{S})$ замкнут. Поэтому достаточно доказать, что каждая точка прикосновения множества $\chi(S)$ принадлежит образу $\chi(\bar{S})$. Но если $\left\{y_{x}\right\}_{x \in E}-$ точка прикосновения $\chi(S)$ и если отображение $T^{\prime}: E \rightarrow E$ определено формулой $T^{\prime} x=y_{x}$ для любых $x \in E$, то $T^{\prime} \in \bar{S}$ по определению слабой операторной топологии. Итак, $\left\{y_{x}\right\}_{x \in E}=\chi\left(T^{\prime}\right) \in \chi(\bar{S})$, что завершает доказательство леммы 1 .

Лемма 2. Пусть $S$ - почти периодическая полугруппа операторов в локально выпуклом пространстве E. Тогда замыкание $\bar{S}$ полугруппы $S$-компактная полутопологическая полугруппа относительно сильной операторной топологии. Если эта полугруппа $S$ является алгебраически группой, то $S$ - компактная топологическая группа относительно сильной ( и слабой) операторной топологuи.

ДокАЗАТЕЛЬСТВо. Повторяя доказательство леммы 1 для сильной топологии, видим, что замыкание $\Sigma$ полугрупшы $S$ в сильной операторной топологии компактно. Кроме того, множество $\Sigma$ компактно и в слабой операторной топологии. Так как сильная операторная топология не слабее слабой операторной топологии, то $\Sigma \subset \bar{S}$, где $\bar{S}$ - замыкание полугруппы $S$ в слабой операторной топологии (см. доказательство леммы 1). Поэтому $\Sigma=\bar{S}$, и ограничение слабой операторной топологии на множество $\bar{S}$ совпадает с ограничением сильной операторной топологии на то же множество (так как $\bar{S}$ компактно в сильной операторной топологии, то непрерывное тождественное отображение этого компактного пространства $\bar{S}=\Sigma$ в сильной операторной топологии на компактное пространство $\bar{S}$ в слабой операторной топологии является гомеоморфизмом). Это завершает доказательство леммы 2.

Лемма 3. Пусть Е - бочечное локально выпуклое пространство и $\mathscr{L}(E)$ пространство непрерывных линейных операторов в Е. Любое слабо компактное семейство операторов в Е равностепенно непрерывно (в исходной топологии пространства $E$ ).

ДокАЗАТЕльство. Слабо компактное множество очевидным образом слабо ограничено. Остальное следует из теоремы III.4.2 в [195]. 
Следующие две леммы устанавливают связи между почти периодичностью представлений и их матричных элементов, т.е. скалярными свойствами почти периодичности.

Лемма 4. Пусть Е - бочечное локально выпуклое пространство со свойством Крейна, $\mathscr{L}(E)$ - пространство непрерывных линейных операторов в $E, G-$ топологическая группа, и пусть $\pi$ - слабо почти периодическое представление группь $G$ в пространстве E. Функиия

$$
F_{f, x}(g)=f(\pi(g) x), \quad g \in G,
$$

слабо почти периодична для любых $x \in E$ u $f \in E^{*}$.

Иначе говоря, слабо почти периодическое представление в бочечном пространстве скалярно слабо почти периодично.

ДокАЗАТЕльство. По лемме 3 образ $\pi(G)$ группы $G$ равностепенно непрерывен в $\mathscr{L}(E)$. Кроме того, из наличия свойства Крейна следует возможность применения критерия слабой компактности Гротендика в форме, приведенной в [195; IV.11.2]. Пусть $x \in E$ и $f \in E^{*}$. Пусть $g_{m}$ и $h_{n}$ - последовательности элементов групшы $G$. Рассмотрим двойную последовательность

$$
F_{f, x}\left(g_{m} h_{n}\right)=\pi\left(g_{m}\right)^{*} f\left(\pi\left(h_{n}\right) x\right) .
$$

Согласно упражнению 25 в гл. IV в [195], последовательность $\pi\left(g_{m}\right) * f$ равностепенно непрерьвна, поэтому, согласно [195; IV .11.2], повторные пределы совпадают, если они существуют. По критерию Гротендика для слабой почти периодичности функций [100] отсюда следует, что все матричные элементы представления $\pi$ слабо почти периодичны.

Лемма 5. Пусть Е - бочечное локально выпуклое пространство, $\mathscr{L}($ E) - пространство непрерывных линейных операторов в $E, G-$ топологическая группа и $\pi$ - почти периодическое представление группь $G$ в пространстве $E$. Функиия $g \mapsto f(\pi(g) x), g \in G$, почти периодична для любих $x \in E$ u $f \in E^{*}$.

Иначе говоря, почти периодическое представление в бочечном пространстве скалярно почти периодично.

ДокАЗАТЕльство. По лемме 3 образ $\pi(G)$ группы $G$ равностепенно непрерывен в $\mathscr{L}(E)$. Поэтому для любой окрестности нуля $U$ в $E$ сушествует такая окрестность нуля $V$ в $E$, что $\pi(g) V \subset U$ для всех $g \in G$. Пусть $\left\{g_{1}, \ldots, g_{n}\right\}$ - такой конечный набор элементов групшы $G$, что

$$
\pi(G) x \subset \bigcup_{i=1}^{n}\left(\pi\left(g_{i}\right) x+V\right)
$$

Тогда

$$
\begin{aligned}
F(h g)=f(\pi(h g) x) & =f\left(\pi(h) \pi(g) x \in \pi(h) \bigcup_{i=1}^{n}\left(\pi\left(g_{i}\right)+V\right)\right) \\
& \subset f\left(\bigcup_{i=1}^{n}\left(\pi\left(h g_{i}\right) x+U\right)\right) \subset \bigcup_{i=1}^{n}\left(F\left(h g_{i}\right)+F(U)\right),
\end{aligned}
$$


так что семейство сдвигов числовой функции $F$ вполне ограничено в топологии равномерной сходимости на групе $G$. Следовательно, $F$ почти периодична.

Следуя Эберлейну [75], Якобсу [115], [116] и де Лю и Гликсбергу [67], введем стандартные эргодические понятия рассматриваемой теории в форме, которую эти понятия приняли в [67] (для случая банаховых пространств).

Напомним, что $\overline{\mathscr{O}(x)}, x \in E,-$ слабое замыкание орбиты $\mathscr{O}(x)$, т.е.

$$
\overline{\mathscr{O}(x)}=\{T x \mid T \in \bar{S}(x)\}
$$

(где черта означает замькание в слабой топологии на пространстве $E$ ).

ОПРЕДЕЛЕНИЕ 4. Вектор $x \in E$ назьвается возвращаемым, если $x \in \overline{\mathscr{O}(y)}$ для любых $y \in \overline{\mathscr{O}(x)}$, или, что то же, если $\overline{\mathscr{O}(x)}=\overline{\mathscr{O}(y)}$ для любых $y \in \overline{\mathscr{O}(x)}$. Вектор $x \in E$ называется исчезающим, если замыкание $\overline{\mathscr{O}(x)}$ орбиты вектора $x$ содержит нулевой вектор $0_{E}$ пространства $E$. Вектор $x \in E$ назьвается компактным, если орбита $\mathscr{O}(x)$ принадлежит конечномерному подпространству, инвариантному относительно $S$, и ограничение полугруппы $S$ на это подпространство является подполугруппой ограниченной групшы линейных операторов на этом подпространстве (причем единичньй элемент группы есть единичньй оператор на этом подпространстве).

Пусть $E_{r}-$ множество всех возврашаемых векторов. Назовем множество $E_{r}$ возвращаемым подмножеством Е. Если $E_{r}$ - подпространство, будем назьвать его возвращаемым подпространством $E$.

Пусть $E_{v}$ - множество всех исчезающих векторов. Пусть $E_{a f d}-$ замыкание линейной оболочки конечномерных векторов в $E$. Элементы подпространства $E_{a f d}$ назовем аппроксимативно конечномерными векторами.

Очевидно, что множество $E_{r}$ и векторное подпространство $E_{a f d}$ являются $\bar{S}$-инвариантными множествами.

Лемма 6. Пусть $S$ - почти периодическая полугруппа операторов в локально выпуклом пространстве $Е$. Пусть $x \in E$.

(1) Следующие условия равносильны:

(1a) $x \in E_{r}$,

(1b) для любого әлемента $T \in \bar{S}$ существует такой әлемент $T^{\prime} \in \bar{S}$, чmо $T^{\prime} T x=x$,

(1c) существует такой идемпотент (проектор) $е \in K(\bar{S})$, что ех $=x$.

(2) Следующие условия равносильны:

(2a) $x \in E_{v}$,

(2b) $T x=0_{E}$ для некоторого $T \in \bar{S}$,

(2c) существует такой идемпотент (проектор) $е \in K(\bar{S})$, что ех $=0_{E}$.

(3) Eсли $\bar{S}$ допускает среднее на всем пространстве $\mathrm{CB}(\bar{S})$ и если е является единственным идемпотентом (проектором) в $K(\bar{S})$ (см. утверждение (2) в теореме В), то еE $\subset E_{a f d}$.

(4) Любой компактный вектор в Е принадлежит $E_{r}$. 
ДокАЗАТЕльство. Эквивалентность $(1 \mathrm{a}) \Longleftrightarrow(1 \mathrm{~b})$ очевидна, так как $\overline{\mathscr{O}(x)}=\{t x \mid$ $t \in \bar{S}(x)\}$ (как правило, мы теперь будем обозначать элементы замькания $\bar{S}$ строчными буквами). Если (1b) выполнено, то пересечение $W=\{t \mid t \in \bar{S}, t x=x\} \cap$ $K(\bar{S})$ непусто, так как $K(\bar{S})$ - левый идеал в $\bar{S}$, а $W$ - компактная подполугруппа в $S$. Согласно утверждению (1) теоремы В, $W$ содержит идемпотент, что доказывает (1c). Итак, (1b) $\Longrightarrow(1 \mathrm{c})$. Если $e$ - идемпотент, удовлетворяюший условию (1c), то $J=\{t e \mid t \in \bar{S}\}$ - минимальньй левьй идеал в $S$ согласно пункту (1а) в теореме В. Если $t \in \bar{S}$, то $t e \in J$; следовательно, существует такой $t^{\prime} \in J$, что $t^{\prime} t e=e$ (см. (1c) в теореме В). Тогда $t^{\prime} t x=t^{\prime} t e x=e x=x$. Итак, (1c) $\Longrightarrow(1 \mathrm{~b})$.

Эквивалентность $(2 \mathrm{a}) \Longleftrightarrow(2 \mathrm{~b})$ очевидна, так как $\overline{\mathscr{O}(x)}=\{t x \mid t \in \bar{S}(x)\}$. Ясно, что $(2 \mathrm{c}) \Longrightarrow(2 \mathrm{~b})$. Так как множество $\ell=\left\{t \mid t \in \bar{S}, t x=0_{E}\right\}$ является левьм идеалом в $\bar{S}$, то из утверждения (1d) в теореме В следует, что $\ell$ содержит идемпотент, принадлежаший ядру $K(\bar{S})$, что доказьвает импликацию $(2 \mathrm{~b}) \Longrightarrow(2 \mathrm{c})$.

Если $\bar{S}$ допускает среднее на пространстве $\mathrm{CB}(\bar{S})$, то $\bar{S}$ имеет только один левый минимальньй идеал и только один правый минимальньй идеал, и оба эти идеала совпадают с $K(\bar{S})$ (см. утверждение (2b) в теореме В). Согласно утверждению (1a) теоремы В, в $K(\bar{S})$ имеется только один идемпотент (проектор) $e$. Ввиду утверждений (1a), (1d) и (2c) теоремы В, ядро $G=K(\bar{S})$ совпадает с минимальным левым идеалом $\ell_{e}=\{t e \mid t \in K(\bar{S})\}=\{t e \mid t \in \bar{S}\}$, и $G$ - компактная топологическая группа с единичным элементом $e$.

Итак, построена некоторая компактная топологическая группа $G$. Пусть $\psi_{\alpha}-$ ограниченная аппроксимативная единица в инволютивной банаховой алгебре $L^{1}(G)$, и пусть эта сеть $\psi_{\alpha}$ образована линейными комбинациями матричных элементов неприводимых конечномерных унитарных представлений группы $G$ (см., например, $[171 ;$ гл. IV,$\S 3.3])$. Введем нормированную меру Хаара $\mu$ на $G$. Для любой функции $\psi_{\alpha}$ положим

$$
t_{\alpha} x=\int_{G} \psi_{\alpha}(t) t x d \mu(t), \quad x \in E,
$$

в смысле формулы (12) приложения 2. Из предложения 1 приложения 2 следует, что если локально вьпуклое пространство $E$ имеет свойство Крейна в смысле определения 12 приложения 1 (в частности, если $E$ квазиполно), то интеграл в (2) существует как элемент пространства $E$. Кроме того, из предложения 2 приложения 2 следует, что если $E$ - пространство Макки (в частности, если $E$ бочечно, см. теорему IV.3.4 в [195]), то формула (2) определяет непрерывный линейный оператор $t_{\alpha}$ в $E$ для любых $\alpha$ (т.е. для любых тригонометрических многочленов $\alpha$ ).

Теперь вернемся к доказательству утверждения (3) леммы 6. Заметим, что

$$
\lim _{\alpha} t_{\alpha} x=e x \quad \text { (слабо) для любых } x \in E .
$$

Действительно, так как $\psi_{\alpha}$ является аппроксимативной единицей в $L^{1}(G)$ и $t \mapsto F(t x)$, $t \in G,-$ непрерьвная ограниченная функция, то можно перейти к пределу в формуле (3) по $\alpha$ следуюшим образом:

$$
F\left(t_{\alpha} x\right)=\int_{G} \psi_{\alpha}(t) F(t x) d \mu(t) \rightarrow F(e x), \quad x \in E, \quad F \in E^{*}
$$


Напомним, что по определению векторное подпространство $E_{a f d}$ (сильно) замкнуто в $E$. Поэтому $E_{a f d}$ слабо замкнуто. Так как $t_{\alpha} x \rightarrow e x$ слабо (согласно (4)), то мы можем завершить доказательство леммы, показав, что $t_{\alpha} x \in E_{a f d}$ для любых $\alpha$.

Возьмем произвольный элемент $x \in E$. Если $N$ - конечномерное векторное подпространство в $\mathrm{C}(G)$, то, так как интегрирование является линейным отображением, отсюда следует, что множество

$$
M_{N}=\left\{\int_{G} f(t) t x d \mu(t) \mid f \in N\right\}
$$

тоже является конечномерньм векторньм подпространством в $\mathrm{C}(G)$. Предположим, что векторное подпространство $N$ инвариантно относительно левых сдвигов на $\mathrm{CB}(S)$. Докажем, что подпространство $M_{N}$ является $S$-инвариантным. Действительно, если $s \in S$ и $s^{\prime}=s e$, то $s^{\prime} \in G$, и $s t=s^{\prime} t$ для любых $t \in G$, так как $е$ является единичным элементом в $G$. Поэтому из левой инвариантности интеграла следует, что

$$
s \int_{G} f(t) t x d \mu(t)=\int_{G} f(t) s^{\prime} t x d \mu(t)=\int_{G} f\left(\left(s^{\prime}\right)^{-1} r\right) r x d \mu(r)
$$

причем правая часть (5) принадлежит $M_{N}$. Формула (5) с $s=e$ показывает, что отображение $е$ оставляет на месте каждый вектор в $M_{N}$.

Докажем, что из инвариантности подпространства $N$ относительно левых сдвигов следует, что подпространство $M_{N}$ является пространством представления конечномерного подпредставления тождественного представления $S$. Заметим, что $e$ действует на $M_{N}$ как единичный оператор, причем $s y=s e y$ для любых $s \in S$ и $y \in M_{N}$. Так как se $\in G$, то ограничение $\left.S\right|_{M_{N}}$ принадлежит ограничению $\left.G\right|_{M_{N}}$ группы $G$ на векторное подпространство $M_{N}$. Это завершает доказательство утверждения (3) леммы 6 , так как $x \in E$ произволен и любой элемент вида $t_{\alpha} x$ принадлежит некоторому подпространству вида $M_{N}$, а мы только что убедились, что эти инвариантные подпространства образованы конечномерными векторами и поэтому принадлежат $H_{a f d}$.

Чтобы доказать утверждение (4), предположим, что $x$ - компактный вектор и $N$ - такое конечномерное подпространство, инвариантное относительно $S$, что ограничение представления $S$ на $N$ определяет подполугруппу ограниченной групшы $G$ линейных операторов на этом подпространстве (и единичньй элемент $e$ группы $G$ действует как единичный оператор на $N)$. В этом случае ограничение полугруппы $\bar{S}$ на $N$ содержится в замькании $\bar{G}$ группы $G$ и $\bar{G}$ - компактная топологическая группа. Так как компактная подполугруппа компактной топологической групшы является (компактной) подгруппой (следствие 2.6 в $[67])$, то $\left.\bar{S}\right|_{N}-$ подгруппа групшы $\bar{G}$. В силу эквивалентности $(1 \mathrm{~b}) \Longleftrightarrow(1 \mathrm{a})$ получаем $N \subset E_{r}$. Это завершает доказательство леммы 6 .

Продолжим изучение слабо компактных полугрупп операторов в локально выпуклом пространстве.

ОПРЕДЕлЕНИЕ 5 . Пусть $S$ - слабо относительно компактная полугруппа операторов в локально выпуклом пространстве $E$. Обозначим через $\mathrm{C}_{E}(S)$ равномерно замкнутую инволютивную подалгебру в $\mathrm{CB}(S)$, порожденную постоянными функциями и 
всевозможными матричными элементами тождественного представления полугруппы $S$, т.е. функциями вида

$$
f(t)=F(t x), \quad t \in S, \quad x \in E, \quad F \in E^{*} .
$$

Алгебра $\mathrm{C}_{E}(S)$ называется представляющей алгеброй операторной полугрупшы $S$ в $E$.

Tеорема F (ср. [67; лемма 4.8]). Пусть $S$ - слабо относительно компактная операторная полугруппа в локально выпуклом пространстве $E, \bar{S}$ - замыкание множества $S$ в пространстве $\mathscr{L}(E)$, снабженном слабой операторной топологией (т.е. топологией простой сходимости в слабой топологии пространства $E$, ср. теорему D выше), и $: S \rightarrow \bar{S}$ - отображсение вложсения. Тогда при отображении ८*: $\mathrm{C}(\bar{S}) \rightarrow \mathrm{CB}(S)$, заданном формулой

$$
\iota^{*}(f)=f \circ \iota, \quad f \in \mathrm{C}(\bar{S}),
$$

пространство $\mathrm{C}(\bar{S})$ отображсается на $\mathrm{C}_{E}(S)$.

ДокАЗАТЕльство. Доказательство практически дословно совпадает с доказательством леммы 4.8 в [67] (относящейся к случаю полугрупп в банаховом пространстве), так как по существу в нем не используется техника банаховых пространств.

Лемма 7 (ср. [67;4.9-4.11]). Пусть E - бочечное пространство со свойством Крейна и $S$ - слабо почти периодическая операторная полугруппа на $E$. Тогда следующие условия әквивалентны:

(1) $C_{E}(S)$ имеет двусторонне инвариантное среднее;

(2) ядро $K(\bar{S})$ - компактная топологическая группа;

(3) ядро $K(\bar{S})$ содержит единственный идемпотент;

(4) $E_{v}$ - замкнутое $S$-инвариантное подпространство в $E$ u $E_{a f d}=E_{r}$, причем $E$ является прямой суммой своих замкнутых $S$-инвариантных подпространств $E_{v}$ u $E_{r}$.

ДокАЗАТЕльство. Эквивалентность $(2) \Longleftrightarrow(3)$ следует из теоремы В. Эквивалентность $(1) \Longleftrightarrow(2)$ следует из леммы 5 и теоремы В.

Если условие $(4)$ вьполнено, то $E_{v}$ - замкнутое векторное подпространство в $E$. Следовательно, $E_{v}$ слабо замкнуто. Так как $E_{v}$ является $S$-инвариантным по предположению, то оно и $\bar{S}$-инвариантно. Пусть $e_{1}$ и $e_{2}-$ идемпотенты в $K(\bar{S})$. Ясно, что $e_{2}\left(1_{E}-e_{2}\right) x=0_{E}$ для любых $x \in E$. Поэтому $\left(1_{E}-e_{2}\right) x \in E_{v}$ для любых $x \in E$. Так как векторное пространство $E_{v}$ является $\bar{S}$-инвариантным, то $e_{1}\left(1_{E}-e_{2}\right) x \in B_{v}$ для любых $x \in E$. Но $e_{1}\left(e_{1}\left(1_{E}-e_{2}\right) x\right)=e_{1}\left(1_{E}-e_{2}\right) x$ для любых $x \in E$, так как $e_{1}-$ идемпотент в $K(\bar{S})$. Поэтому $e_{1}\left(1_{E}-e_{2}\right) x \in E_{r}$ для любых $x \in E$ согласно утверждению (1) (см. (1c) и (1a)) в лемме 6. Из определения множеств $E_{r}$ и $E_{v}$ сразу следует, что $E_{r} \cap E_{v}=\left\{0_{E}\right\}$. Таким образом, $e_{1}\left(1_{E}-e_{2}\right) x=0_{E}$ для любых $x \in E$, и это доказывает, что

$$
e_{1} e_{2}=e_{1}
$$

для любых двух идемпотентов $e_{1}, e_{2} \in K(\bar{S})$. 
Воспользуемся теперь условием, что $E_{a f d}=E_{r}$ и $E_{r} \cap E_{v}=\left\{0_{E}\right\}$. Если $e-$ идемпотент в $\bar{S}$ и $x \in E-$ компактньй вектор, то $x \in E_{a f d}=E_{r}$. Так как $e x \in E_{r}$ по лемме 6 (см. эквивалентность (1c) $\Longleftrightarrow(1 \mathrm{a})$ ), отсюда следует, что $x-e x \in E_{r}$, поскольку $E_{r}=E_{a f d}-$ векторное пространство. Так как $e$-идемпотент, мы видим, что $e(x-e x)=0_{E}$. Поэтому $x \in E_{v}$ по лемме 6 (см. эквивалентность $\left.(2 \mathrm{c}) \Longleftrightarrow(2 \mathrm{a})\right)$. Так как $E_{r} \cap E_{v}=\left\{0_{E}\right\}$ по предположению, отсюда следует, что $e x=x$ для любых компактных векторов $x$ и любого идемпотента $e \in \bar{S}$. Следовательно, любой идемпотент $e \in \bar{S}$ оставляет на месте каждый элемент пространства $E_{a f d}=E_{r}$, в котором множество компактных векторов тотально. Если $e_{1}$ и $e_{2}-$ идемпотенты в $K(\bar{S})$ и $x \in E$, то $e_{2} x \in E_{r}$ снова по лемме 6 (см. эквивалентность $\left.(1 \mathrm{c}) \Longleftrightarrow(1 \mathrm{a})\right)$, и поэтому $e_{2} x \in E_{a f d}$ в силу предположения $E_{a f d}=E_{r}$. Так как $e_{1}$ оставляет неподвижным каждый элемент подпространства $E_{r}$, то $e_{1} e_{2} x=e_{2} x$ для любых $x \in E$, и поэтому

$$
e_{1} e_{2}=e_{2}
$$

для любых идемпотентов $e_{1}, e_{2} \in K(\bar{S})$. Объединяя $(7)$ и (8), получаем равенство $e_{1}=e_{2}$ для любых идемпотентов $e_{1}, e_{2} \in K(\bar{S})$. Это завершает доказательство импликации $(4) \Longrightarrow(3)$.

Докажем теперь, что (3) $\Longrightarrow(4)$. Пусть выполнено условие (3), и пусть $e-$ идемпотент в $K(\bar{S})$. Если $x \in E$, то $e\left(1_{E}-e\right) x=0_{E}$, и поэтому $\left(1_{E}-e\right) x \in E_{v}$. С другой стороны, множество $E_{r}-$ это множество всех таких векторов $x \in E$, что $e x=x$ (см. эквивалентность (1c) $\Longleftrightarrow(1 \mathrm{a})$ в лемме 6$)$. Поэтому $E_{r}$ - замкнутое векторное подпространство в $E$. Любой компактньй вектор принадлежит $E_{r}$ по утверждению (4) в лемме 6. Таким образом, $E_{a f d} \subset E_{r}$. Но $(3) \Longleftrightarrow(2)$, и из утверждений (1) и (3) в лемме 6 следует, что $E_{r} \subset E_{a f d}$. Таким образом, $E_{a f d}=E_{r}$, и формула $x=e x+$ $\left(1_{E}-e\right) x \in E_{r} \dot{+} E_{v}$ дает требуемое разложение, так как $E_{r} \cap E_{v}=\{0\}$ согласно соотношению $e\left(1_{E}-e\right) x=0_{E}$, справедливому для любых $x \in E$. Это завершает доказательство леммы 7 .

Обобщения приведенных выше результатов на случай представлений в локально вьпуклых пространствах Макки приведены в [203].

\section{§ 4. Связь между почти периодичностью, слабой почти периодичностью и скалярной почти периодичностью}

Одна из общих задач теории почти периодических представлений и функций в локально вьпуклых пространствах состоит в том, чтобы найти условия почти периодичности слабо почти периодического представления или функции со значениями в локально вьпуклом пространстве. Это - самый продуктивньй тип связей между важнейшими классами почти периодических функций (между сильной, скалярной и слабой почти периодичностью). В этом направлении известны только результаты о банаховых пространствах; см. упомянутую вьше книгу Ю.И. Любича [147] и последующие статьи М.И. Кадеца и Любича [125] и М.И. Кадеца [122]; часть этих результатов отражена в книге Б. М. Левитана и В.В. Жикова [141]. Читатель легко заметит, что даже в статьях [122] и [125] условия слабой почти периодичности представления включают требование сильной непрерьвности рассматриваемых представлений. Как мы увидим, это условие избыточно в связи с явлением автоматической сильной 
непрерьвности представлений рассматриваемого типа и поэтому может быть заменено формально более слабым условием слабой непрерьвности. Более того, оказывается возможным (при определенных условиях) распространить соответствующий результат на случай представлений в локально вьпуклых пространствах, что потребует обобщения аппарата де Лю и Гликсберга [67] на локально вьпукльй случай.

Поскольку теорема Кадеца и Любича [125] (уточняюшая результат, приведенный в книге Любича [147] для случая рефиексивных банаховых пространств) является моделью нашего будущего критерия почти периодичности слабо непрерьвных слабо почти периодических представлений в бочечных пространствах со свойством Крейна, мы приведем формулировку основного утверждения в [125].

Теорема G [125]. Пусть $\pi$ - сильно непрерывное представление топологической группь $G$ в банаховом пространстве $E$. Представление $\pi$ является почти периодическим (и, таким образом, замыкание по норме каждой орбить $\mathscr{O}(x)=\{\pi(g) x \mid g \in G, x \in E\}$ компактно в $E$ в сильной топологии пространства Е) тогда и только тогда, когда $\pi$ слабо почти периодично (т.е. все орбить $\mathscr{O}(x), x \in E$, относительно слабо компактны в $E)$ и матричные әлементы представления $\pi$ являются комплексными почти периодическими функциями на $G$.

Авторы приводят пример, показываюший, что условие слабой почти периодичности существенно в утверждении “тогда". Этот контрпример использует более мощные средства, чем стандартный пример, показывающий, что условие слабой секвенциальной полноты (или отсутствие подпространства в $E$, изоморфиного пространству $c_{0}$ стремящихся к нулю последовательностей) для банахова пространства $E$ существенно для справедливости знаменитой теоремы Люмиса ([145]; см. также [44], [125]), утверждаюшей, что при этих условиях ограниченная непрерьвная $E$-значная функция с не более чем счетньм спектром [6], [12], [16], [17], [27], [28], [50], [105], [218] является почти периодической.

Мы рассмотрим только этот стандартный пример, кочующий из одной статьи в другую и ставший частью математического фольклора. Рассмотрим представление аддитивной групшы $\mathbb{R}$ вешественных чисел в пространстве $c$ (всех сходяшихся последовательностей), заданное формулой $\pi(t) e_{n}=\exp (t / n) e_{n}$, где $t \in \mathbb{R}$ и $n \in \mathbb{N}$. Ясно, что представление $\pi$ имеет почти периодические матричные элементы, поскольку все эти матричные элементы являются суммами рядов вида

$$
\sum_{n=1}^{\infty} c_{n} \exp (t / n), \quad t \in \mathbb{R}, \quad \text { где ряд } \sum_{n} c_{n} \text { абсолютно сходится. }
$$

Но представление $\pi$ не является почти периодическим, так как орбита точки $(1,1, \ldots)$ не является (сильно) компактным множеством в $c$. В самом деле, если бы эта орбита была компактна, то последовательность функционалов $\varphi_{n}(x)=x_{n}, x=\left\{x_{n}\right\} \in c$, стремилась бы к 1 на каждом элементе орбиты при $n \rightarrow \infty$ (так как последовательность $\exp (t / n)$ стремится к 1 поточечно), но эта сходимость не является равномерной на орбите. Следовательно, орбита точки $(1,1, \ldots)$ не является (сильно) компактной в $c$. Это доказьвает, что $\pi$ не является почти периодическим представлением. Из самой теоремы Кадеца и Любича следует, что это представление не является и слабо почти периодическим. 
Как показано в [18], если $f$ - функция на топологической группе $G$ со значениями в пространстве $\Phi$ реше $A$, если при $\gamma \in G$ функция $\Delta_{\gamma} f$ определена формулой $\Delta_{\gamma} f(t)=f(t \gamma)-f(t), t \in G$, и если функция $\Delta_{\gamma} f$ непрерьвна в единичном элементе $e$ групшы $G$ для любых $\gamma$ в некоторой окрестности точки $e$, то функция $f$ непрерывна в $e$ в предположении, что $A$ не содержит подпространства, изоморфного $c_{0}$, и $f$ ограничена. Аналогичньй результат доказан там и в случае, если $A$ - локально вьпуклое пространство и область значений функции $f$ слабо относительно компактна. Несмотря на ряд результатов о свойствах локально вьпуклых пространств, не содержаших подпространства, изоморфного $c_{0}$ (типичным является результат в [73]), условия слабой секвенциальной полноты пространства, не содержащего подпространства, изоморфного $c_{0}$, получены в локально выпуклом случае только для пространств с безусловньм базисом. По этой причине мы не пытаемся распространить $c_{0}$-результат теории почти периодических функций в банаховых пространствах на локально выпукльй случай и ограничиваемся обобшением и уточнением качественного подхода Кадеца и Любича $[125]$.

Приведем анонсированньй выше результат.

ТЕОРема 1. Пусть $\pi$ - слабо непрерьвное представление топологической группы $G$ в бочечном локально выпуклом пространстве Е со свойством Крейна. Представление $\pi$ почти периодично тогда и только тогда, когда $\pi$ слабо почти периодично и скалярно почти периодично. Кроме того, если эти равносильнье условия выполнены, то представление $\pi$ сильно непрерывно.

Таким образом, для каждой орбиты $\mathscr{O}(x)=\{\pi(g) x \mid g \in G\}, x \in E$, слабо непрерьвного представления $\pi$ топологической группы $G$ в бочечном локально вьпуклом пространстве $E$ со свойством Крейна сильное замыкание этой орбиты компактно в $E$ в сильной топологии тогда и только тогда, когда все орбиты $\mathscr{O}(x), x \in E$, относительно слабо компактны в $E$ и все матричные элементы представления $\pi$ являются комплексными почти периодическими функциями на $G$.

ДокАЗАТЕЛЬСТво. Если представление является почти периодическим, то это представление очевидным образом является и слабо почти периодическим. Скалярная почти периодичность (почти периодичность матричных элементов) следует из леммы 5 . Поэтому остается доказать обратную импликацию.

Пусть $\pi$ - слабо непрерывное представление топологической группы $G$ в бочечном пространстве $E$ со свойством Крейна (см. определение 12 в приложении 1). Предположим, что $\pi$ слабо почти периодично (т.е. слабое замыкание каждой орбиты $\mathscr{O}(x)=\{\pi(g) x \mid g \in G\}, x \in E$, компактно в $E$ в слабой топологии). По лемме 1 , замыкание $S$ образа $\pi(G)$ группы $G$ в представлении $\pi$ в слабой операторной топологии на $\mathscr{L}(E)$ есть компактная полутопологическая полугруппа операторов в слабой операторной топологии относительно обычного умножения операторов. Объединяя утверждения теорем С и $\mathrm{D}$, видим, что представляющая алгебра $\mathrm{C}_{E}(\pi(G))$ естественно $G$-эквивариантно изоморфна обеим алгебрам $\mathrm{C}(S)$ и $W A P(S)$, а также некоторой подалгебре алгебры $W A P(G)$ (и притом содержит все постоянные функшии). По теореме Е, алгебра $W A P(G)$ допускает двусторонне инвариантное среднее и, снова по теоремам С и D, алгебра $\mathrm{C}(S)$ допускает (двусторонне) инвариантное среднее. Согласно утверждению (7) теоремы D, алгебра $W A P(S)$ разлагается в прямую сумму двух подпространств, инвариантных относительно сдвигов, а именно 
$W A P(S)_{r}$ и $W A P(S)_{v}$, первое из которых можно отождествить с пространством $A P(S)$, а другое - с подпространством $\left\{f \mid f \in W A P(S), m\left(|f|^{2}\right)=0\right\}$. Но, по предположению, представляющая алгебра является подалгеброй алгебры $A P(G)$. Следовательно, компонента функции, принадлежащая $W A P(S)_{v}$, одновременно принадлежит и $A P(G)$, и $W A P(S)_{v}$. Однако эти пространства имеют тривиальное пересечение, так как сумма подпространств $W A P(S)_{r}$ и $W A P(S)_{v}$ - прямая. Таким образом, $W A P(S)=A P(S)$; это означает, что $W A P(S)_{v}=0$ и $e=1_{E}$. Поэтому полугруппа $S$ совпадает со своим ядром. Следовательно, алгебраически полугруппа $S$ является группой. По теореме Эллиса (теорема A), $S$ - компактная топологическая група. Поэтому замыкание образа $\pi(G)$ в слабой операторной топологии - компактная топологическая група в слабой топологии. По теореме о сильной непрерывности слабо непрерьвных представлений (локально) компактных групп в бочечных пространствах [168] тождественное представление группы $S$ сильно непрерьвно. Следовательно, замыкание множества $\pi(G)$ в сильной операторной топологии компактно. Это показьвает, что рассматриваемое представление почти периодично. Снова используя сильную непрерывность тождественного представления группы $S$, видим, что композиция этого (тождественного) отображения с исходньм слабо непрерьвньм представлением является сильно непрерьвным представлением и его образ принадлежит сильно компактной группе операторов в $E$. Это завершает доказательство теоремы 1.

Обобщение теоремы 1 на случай представлений в локально выпуклых пространствах Макки приведено в [203].

Заметим, что теорема 1 - не единственный путь к автоматической почти периодичности скалярно почти периодических представлений групп и полугрупп. Например, неприводимые слабо почти периодические представления полутопологических полугрупп марковскими операторами на компакте автоматически почти периодичны [118].

\section{$\S$ 5. Родственные результаты и приложения}

\section{1. Критерии почти периодичности и слабой почти периодичности.}

Уточним утверждения лемм 4 и 5 .

ТеОРема 2. Пусть $\pi$ - слабо непрерывное представление топологической группы $G$ в локально выпуклом пространстве $E$. Представление $\pi$ слабо почти периодично тогда и только тогда, когда мнодсество $\pi(G)$ относительно слабо компактно в пространстве $\mathscr{L}(E)$ непрерывных линейных операторов в $E$, снабженном слабой операторной топологией (определяемой системой полунорм вида $\left.T \mapsto|f(T x)|, x \in E, f \in E^{*}\right)$.

ДокАЗАТЕльство. В одну сторону утверждение доказано в лемме 1. Докажем обратное утверждение. Если $\pi(G)$ относительно слабо компактно в пространстве $\mathscr{L}(E)$ непрерьвных линейных операторов в $E$, снабженном слабой операторной топологией, то замькание $S$ множества $\pi(G)$ в пространстве $\mathscr{L}(E)$ является компактной полутопологической полугруппой снова по лемме 1 . Орбиты этой полугруппы слабо компактны в $E$ как непрерывные образы компакта $S$ и содержат соответствующие орбиты представления $\pi$, которые поэтому относительно слабо компактны. 
ТЕОРема 3. Пусть $\pi$ - слабо почти периодическое представление топологической группы $G$ в локально выпуклом пространстве Е. Представление $\pi$ почти периодично тогда и только тогда, когда множество $\pi(G)$ относительно компактно в пространстве $\mathscr{L}(E)$ непрерывных линейных операторов в $E$, снабженном сильной операторной топологией (определяемой системой полунорм вида $T \mapsto p(T x)$, где $x \in E$ и выполнено, то представление $\pi$ сильно непрерывно.

ДокАЗАТЕльство. В одну сторону утверждение доказанов лемме 2. Доказательство обратного утверждения аналогично доказательству соответствующей части теоремы 2. Если же эти равносильные условия вьполнены, то доказательство сильной непрерьвности представления $\pi$ повторяет соответствующую часть доказательства теоремы 1 .

\section{2. Среднее на слабо почти периодических функциях.}

Пусть $G$ - топологическая группа, $E$ - бочечное локально вьпуклое пространство со свойством Крейна, $f$ - слабо почти периодическая функция на $G$ со значениями в $E$. Числовые функции $\varphi \circ f$, определенные формулой $\varphi \circ f: g \mapsto \varphi(f(g)), g \in G$, слабо почти периодичны на $G$ для любых $\varphi \in E^{*}$ (это доказывается так же, как и лемма 4 ), и поэтому на каждой из этих функций определено значение $\mu(\varphi \circ f)$, где $\mu$ - (единственное) инвариантное среднее на пространстве числовых слабо почти периодических функций на группе $G$, см. [97]. Нетрудно видеть, что формула $\varphi(m(f))=\mu(\varphi \circ f)$, $\varphi \in E^{*}$, определяет элемент $m(f)$ как (формально - не обязательно непрерьвньй) линейньй функщионал на $E^{*}$ (cp. [90]). Так как среднее $\mu$ содержится в замкнутой выпуклой оболочке точечных функционалов (определяемых элементами $g \in G$ группы $G$ по правилу $\left.\varphi \rightarrow \varphi(f(g)), \varphi \in E^{*}\right)$, а множество значений функции $f$ слабо относительно компактно (как образ слабо относительно компактной орбиты функции $f$ при отображении, определяемом взятием значения функции в единичном элементе, поскольку это отображение непрерывно по определению 3$)$, то элемент $m(f)$ пространства $E^{* \prime}$ линейных функционалов на $E^{*}$ определяется элементом пространства $E$, принадлежашим тому же компактному множеству, а именно замкнутой вьпуклой оболочке слабо компактного замыкания множества значений функции $f$.

Таким образом, мы построили векторнозначное инвариантное среднее $m$ на $W A P(G, E)$ со значениями в $E$. Некоторое видоизменение доказательства банаховой версии утверждения в [230] позволяет установить, что отображение $m$ совпадает с (единственным) линейным отображением пространства $W A P(G, E)$ в $E$, перестановочным с действием групшы сдвигами и переводящим каждую слабо почти периодическую функцию в элемент пространства $E$, принадлежаший замкнутой вьпуклой оболочке ее множества значений.

Средние значения (в различных смыслах) на различных классах почти периодических функций, а также на дистальных функциях, эргодических функциях и функциях более обших классов (в том числе в связи с взаимоотношениями между определениями почти периодических функций по Бору и Бохнеру) рассматриваются во многих работах и имеют многообразные приложения [1], [19], [36], [39], [40], [47], [53], [86], [87], [90], [97], [103], [108], [112], [131], [135], [136], [149], [183], [186], [188], [193], [217], $[228],[229],[233] .^{9}$

\footnotetext{
${ }^{9} \mathrm{~B}[183]$ отмечено интересное явление. Пусть $G=\mathbb{R} \times \mathbb{R}^{+}{ }_{-}$группа афффинных преобразований
} 


\section{3. Условия счетности спектра скалярно почти периодической функ-} ции.

Теорема Бохнера и фон Неймана [43] о счетности спектра функций, почти периодических в смысле фон Неймана (при условиях полноты и сепарабельности пространства, которые напоминаются ниже, в приложении 1), вызвала многочисленные исследования феномена счетности спектра для других классов почти периодических функций с векторными значениями (ср. [123]).

Напомним следующее стандартное определение.

ОПРЕДЕЛЕниЕ 6 . Пусть $G$ - топологическая группа, $\widehat{G}$ - семейство классов эквивалентности неприводимых унитарных представлений группы $G$ в конечномерных гильбертовых пространствах, $E$ - бочечное локально выпуклое пространство со свойством Крейна, $m$ - введенное в п. 5.2 инвариантное среднее на $W A P(G, E)$. Пусть $\omega \in \widehat{G}$ и $P_{\omega}$ - отображение, сопоставляющее слабо почти периодической функции ее $\omega$-компоненту

$$
P_{\omega} f(\cdot)=\operatorname{dim} \omega \cdot \operatorname{trace}\left(m\left(f \cdot(\omega(g))^{-1}\right) \cdot \omega(\cdot)\right) .
$$

Cnектром функции $f \in W A P(G, E)$ называется множество

$$
\sigma(f)=\left\{\omega \in \widehat{G} \mid P_{\omega} f \neq 0\right\} .
$$

Слабым спектром скалярно почти периодической функции $f: G \rightarrow E$ назьвается объединение спектров матричных элементов функции $f$, т.е. числовых почти периодических функций вида $\varphi \circ f, \varphi \in E^{*}$.

Справедлив следуюший критерий счетности спектра скалярно почти периодических функций со значениями в банаховом пространстве [104] (на самом деле этот критерий имеет более развитую форму, но мы ограничиваемся той частью критерия Гюнцлера, понимание которой не требует освоения новых определений, в частности, разработанных в [101], [102]).

Теорема Н (Гюнцлер, [104]). Пусть $S$ - полугруппа, $E$ - банахово пространство и $f: S \rightarrow E-$ скалярно почти периодическая функиия. Следующие условия равносильны:

(1) слабый спектр функции $f$ не более чем счетен,

(2) на образе $f(S)$ слабая топология пространства Е удовлетворяет первой аксиоме счетности,

прямой с обычным умножением $\left(x^{\prime}, y^{\prime}\right)(x, y)=\left(x^{\prime}+y^{\prime} x, y^{\prime} y\right)$. Можно проверить, что функция $f(x, y)=e^{i x}$ не является почти периодической на $G$, но ее правые сдвиги на элементы подгруппы $H=\{0\} \times \mathbb{R}^{+}$определяют предкомпактное семейство; тем самым есть класс таких функций; обозначим его $R A P(G, H)$. Заметим, что $f \notin L A P(G, H)$ (которое определяется аналогично). Следуя [170], оказывается возможным изучить условия справедливости равенств, подобных соотношению $R A P(G, G)=A P(G)$, векторные средние и компактификации. Это исследование показьвает, насколько близко расположены вопросы, касающиеся почти периодических функций и представлений и почти периодических действ ий и динамических систем, и переход с язьпа динамики на язык представлений совершенно естествен, даже если при этом часть информации о системе утрачивается. Теория почти периодических функций и представлений оказывается полезной во многих вопросах топологической динамики; см., например, [1], [37], [44], [77]-[79], [93], [121], [136], [141], [157]-[166], [169], [216], [221], [233], [234]. 
(3) множсество значений функции $f$ сепарабельно,

(4) существует счетная подполугруппа в $S$, замыкание образа которой содержит образ полугруппь $S$.

Ранее, в 1980 г., для группы вещественных чисел в обычной топологии этот результат был анонсирован Кадецом и Кюрстеном [124] с наброском доказательства. Поразительно, но замечательный критерий Гюнцлера (теорема Н) остался почти незамеченньм. В частности, в статье [123] доказано, что если $X$ - такое банахово пространство, что слабое* секвенциальное замыкание каждого его сепарабельного подпространства сепарабельно в его втором сопряженном, то любое скалярно почти периодическое представление групшы вешественных чисел в пространстве $X$ является почти периодическим, причем автор утверждает, что критерий счетности спектра для груп, отличных от вещественной прямой, ему неизвестен. Объединение доказательства Кадеца с критерием Гюнцлера позволяет немедленно получить, например, следующее более общее утверждение.

Теорема 4. Пусть $X$ - банахово пространство. Предположим, что слабое* секвенииальное замыкание кажддого сепарабельного подпространства $Y$ пространства $X$ сепарабельно во втором сопряэсенном $Y^{* *}$. Тогда каждое скалярно почти периодическое представление сепарабельной абелевой локально компактной группы в пространстве $X$ является почти периодическим.

Интересно, верно ли это утверждение для сепарабельных топологических групп более общего вида. Вопрос о справедливости критерия Гюнщлера (теоремы Н) для скалярно почти периодических функций со значениями в более обших локально выпуклых пространствах также представляет естественньй интерес.

Помимо условий счетности спектра, начиная с еще довоенных работ Левитана и Вольфа, изучаются спектральные условия того, что данная ограниченная непрерьвная функция является почти периодической. Кроме Люмиса [145] и Жикова (см. [141]), в формировании современного вида этой теоремы для случая банаховых пространств приняли участие многие авторы, в том числе - Баскаков [27], [28] и Арендт и Швейкер [6]. Для простоты мы ограничимся случаем почти периодических функций на коммутативных локально компактных группах со значениями в банаховых пространстваx.

ОПРЕДЕЛЕНИЕ 7. Пусть $G$ - коммутативная локально компактная група. Cneкmром равномерно непрерьвной функщии $f: G \rightarrow E$, где $E$ - банахово пространство, называется множество $\operatorname{sp}(f)=\left\{\chi \in \widehat{G} \mid(\mathscr{F} \varphi)(\chi)=0\right.$ для всех $\left.\varphi \in I_{f}\right\}$, где $\widehat{G}$ - группа характеров групшы $G, I_{f}=\left\{\varphi \in L^{1}(G) \mid \varphi * f=0\right\}$ и

$$
(\mathscr{F} \varphi)(\chi)=\int_{G} \overline{\chi(s)} \varphi(s) d s
$$

- преобразование Фурье функции $\varphi \in L^{1}(G)$. Спектром Арвесона сильно непрерьвного представления $\pi$ групшы $G$ в $E$ назьвается множество

$$
\operatorname{Sp}(\pi)=\left\{\chi \in \widehat{G} \mid(\overline{\mathscr{F}} \varphi)(\chi)=0 \text { для всех } \varphi \in I_{\pi}\right\},
$$

где

$$
(\overline{\mathscr{F}} \varphi)(\chi)=\int_{G} \chi(s) \varphi(s) d s
$$


и $I_{\pi}=\left\{\varphi \in L^{1}(G) \mid \varphi(S)=0\right\}$ (в смысле представления групповой алгебры, определяемого представлением групшы $G)$.

Множество $A \subset \widehat{G}$ назьвается разреженным, если оно не содержит непустых совершенных подмножеств.

Теорема I [6], [27]. Пусть $G$ - коммутативная топологическая группа, Eбанахово пространство, $\pi$ - слабо почти периодическое представление групnы $G$ со значениями в $E$. Если спектр Арвесона представления $f$ разрежсен, то представление $\pi$ почти периодично.

ДокАЗАТЕЛЬство. В силу теоремы 1 доказательство сводится к проверке почти периодичности матричных элементов представления $\pi$, которая, в свою очередь, следует из известной теоремы о скалярных функциях (см., например, теорему VI.4.4 в [141]).

Аналогичное утверждение справедливо для функций со значениями в банаховых пространствах [6]. Есть и более сильные варианты этих утверждений. Например, для функций со значениями в банаховых пространствах в условии теоремы I можно заменить спектр Арвесона содержащимся в нем "почти периодическим спектром" (см. $[27],[141])$.

Ни одно из сушествующих спектральных понятий не имеет очевидного аналога в локально вьпуклом случае. Впрочем, некоторые разумные утверждения могут быть доказаны (для функций и представлений в локально вьпуклых пространствах) и теми средствами, которые есть в наличии. Мы сформулируем и докажем соответствуюшую теорему в следующем пункте, после того как выясним связь между слабо почти периодическими функциями и компактными операторными полугруппами в случае, когда пространства не нормированы.

\section{4. Слабая почти периодичность функций и представлений.}

Специфика слабо почти периодических функций со значениями в банаховом пространстве состоит в том, что семейство слабо почти периодических функций со значениями в банаховом пространстве есть банахово пространство (теорема 2.5 в [35]). Таким образом, теорема 1 (о разложении слабо почти периодических представлений) может быть непосредственно применена к представлению этой групшы сдвигами в пространстве слабо почти периодических функций со значениями в данном банаховом пространстве, что приводит к аналогичному разложению слабо почти периодических функций в этом пространстве. Это следствие теоремы 1 составляет содержание основной теоремы Чжана (теорема 8.8 в [230]) о разложении пространства слабо почти периодических функций со значениями в банаховом пространстве $E$ в прямую сумму инвариантных замкнутых подпространств, одно из которых образовано почти периодическими функциями со значениями в $E$, а другое - слабо почти периодическими функциями со значениями в $E$, замкнутая вьпуклая оболочка которых содержит функцию, тождественно равную нулю ${ }^{10}$. Но примененные вьше методы анализа представлений в локально выпуклых пространствах не всегда допускают такую итерацию: за пределами пространств Фреше (полных метризуемых локально вьпуклых топологических

\footnotetext{
${ }^{10}$ Ранее Голдберг и Ирвин [90] получили аналогичную теорему разложения в более узком классе сильно непрерывных ограниченных функций на топологической группе со значениями в банаховом пространстве, орбита сдвигов которых в пространстве всех таких функций слабо относительно компактна, а образ функции относительно компактен в смысле нормы пространства.
} 
векторных пространств) условия бочечности пространства, образованного слабо непрерьвньми функциями на вполне регулярном пространстве (и даже на бесконечном компакте) со значениями в бочечном пространстве и рассматриваемого в топологии сильной равномерной сходимости, неизвестны.

Отметим здесь, что структура пространства $W C(K, X)$ слабо непрерывных функций на компактном топологическом пространстве $K$ со значениями в банаховом пространстве $X$ (с обычной равномерной нормой) отнюдь не проста и в ряде других отношений [7]. Любая функция $f \in W C(K, X)$ измерима по Борелю и $\mu$-интегрируема по Бохнеру относительно любой конечной регулярной борелевской меры $\mu$ на $K$. Пространство $\mathrm{C}(K, X)$ сильно непрерывных функций на $K$ со значениями в $X$ образует замкнутое подпространство в $W C(K, X)$, причем $W C(K, X) \subseteq \mathrm{C}(K, X)^{* *}$ и $\mathrm{C}(K, X)^{*}$ дополняемо в $W C(K, X)^{*}$. Известно также, что подпространство $\mathrm{C}(K, X)$ дополняемо в $W C(K, X)$ тогда и только тогда, когда эти пространства совпадают. Если пространство $X$ имеет свойство Шура (т.е. слабая и сильная сходимости последовательностей совпадают, или, что то же, условия слабой и сильной компактности подмножеств пространства $X$ совпадают), то $\mathrm{C}(K, X)-M$-идеал в $W C(K, X)$ (напомним, что подпространство $F$ банахова пространства $E$ называется $M$-идеалом в $E$, если его аннулятор $F^{\perp}$ в сопряженном пространстве $E^{*}$ является образом $L$-проектора $P: E^{*} \rightarrow F^{\perp}$, т.е. $P^{2}=P$ и $\|P x\|+\|x-P x\| \equiv x$ для любого $\left.x \in E^{*}\right)$. Поразительно, но сами по себе перечисленные выше свойства измеримости и интегрируемости слабо непрерьвных функщий (без дополнительных геометрических условий на банахово пространство) не обеспечивают интегрируемости нормы функции по Риману. А именно, банахово пространство $X$ имеет свойство Шура (заметим, что каждая скалярно почти периодическая функщия со значениями в таком пространстве автоматически является почти периодической) тогда и только тогда, когда для любой слабо непрерьвной функции $f:[0,1] \rightarrow X$ функция $\|f(\cdot)\|$ интегрируема по Риману на $[0,1]$ (cм. $[7])$.

Крайние точки единичного шара $B\left(W C(K, X)^{*}\right)$ в $W C(K, X)^{*}$ суть точечные функционалы; более точно, такие функционалы $\theta$ имеют вид $\theta(f \cdot a)=f\left(k_{0}\right) \cdot \theta(a)$ для всевозможных $f, a \in W C(K, X)$ и $k_{0} \in K[7]$.

Перейдем к основньм утверждениям. Прежде всего, мы располагаем следующим обобщением теоремы Чжана на случай функций со значениями в пространствах $\Phi$ реше. (Для сильно непрерьвных функций ситуация несколько лучше, см. теорему 6 ниже.)

ТеОрема 5. Пусть $G$ - топологическая группа, E- пространство Фреше. Пространство $W A P(G, E)$ слабо почти периодических функций на $G$ со значениями в Е допускает разложение в прямую сумму двух инвариантных замкнутых векторных подпространств, одно из которых образовано почти периодическими функииями на $G$ со значениями в $E$, а другое - всеми сильно равномерно непрерывными на группе $G$ слабо почти периодическими функциями на $G$ (со значениями в $E$ ), замкнутая выпуклая оболочка которых содержит функиию, тождественно равную нулю.

ДоказАТЕльство. Пространство $W A P(G, E)$ является пространством Фреше относительно естественной "равномерной” метрики. Пусть $F \in W A P(G, E)$, т.е. орбита $F$ слабо относительно компактна в пространстве слабо непрерывных $E$-значных 
функций на $G$ с равномерной метрикой. Как и в лемме 4 , из критерия Гротендика непосредственно следует, что для любого $f \in E^{*}$ функция $F_{f}(g)=f(F(g)), g \in G$, является числовой слабо почти периодической функцией на $G$. Любая непрерьвная слабо почти периодическая функция на $G$ с числовыми значениями допускает единственное продолжение до непрерьвной функции на компактной полутопологической полугруппе $G^{\mathscr{W} \mathscr{A P}}$, так называемой слабо почти периодической компактификации группы $G$ (см. теорему D в $\S 2$ ). Нетрудно проверить, что семейство значений продолжений всевозможных матричных элементов $F_{f}$ определяет в каждой точке полугрупшы $G^{\mathscr{W A} \mathscr{A P}}$ некоторый элемент пространства $E^{* \prime}$ (не обязательно непрерывных) линейных функционалов на $E^{*}$. Используя по существу те же рассуждения, что и в начале п. 5.2, видим, что каждый из этих функционалов определяется некоторым элементом пространства $E$ и содержится в слабо замкнутой вьпуклой оболочке слабо относительно компактного множества (образа функции $F$ в $E$ ). Это построение определяет функцию $\Phi: G^{\mathscr{W} \mathscr{A P}} \rightarrow E$, ограничение которой на $G$ совпадает с $F$, причем, как и в [198], отсюда следует, что функция $\Phi$ слабо непрерывна на $G^{\mathfrak{W} \mathscr{A} \mathscr{P}}$. Поэтому орбита функции $\Phi$ в (как легко проверить, полном) метрическом пространстве слабо непрерьвных функций $W C\left(G^{\mathscr{W} \mathscr{A P}}, E\right)$ (с естественной равномерной метрикой) слабо компактна. Следовательно, орбита функции $F$ в полном метрическом пространстве (слабо непрерьвных) слабо почти периодических функций $W A P(G, E)$ (с равномерной метрикой) относительно слабо компактна. Таким образом, представление группы $G$, определяемое сдвигами в пространстве $W A P(G, E)$, слабо почти периодично. Остается применить теорему 1 .

Нетрудно сформулировать и доказать и аналогичное утверждение для сильно непрерьвных функций.

Это - многообещающие утверждения. Они открьвают путь к изучению почти периодических функций со значениями в пространствах голоморфных функций и некоторых пространствах обобщенных функций (в духе исследования почти периодических мер, см., например, [88]). Конечно, пространства обобшенных функций чаще оказываются пространствами типа (DF), чем типа (F), и редко бывают рефлексивными. Но сильное сопряженное к пространству Фреше бьвает бочечным и за пределами класса рефлексивных пространств. Поэтому существенно, что с помощью критерия бочечности пространств непрерьвных функций на компакте со значениями в бочечном пространстве [156] можно, хоть и дорогой ценой, несколько продвинуться за пределы метризуемой ситуашии. Перейдем к формулировке и доказательству соответствующего утверждения.

Начнем со следуюшей леммы, которая объясняет, почему произведение локально равностепенно непрерьвных сильно непрерывных операторнозначных функций сильно непрерывно, если речь идет о бочечном пространстве.

Лемма 8. Пусть $E$ - бочечное локально выпуклое пространство, $\mathcal{L}(E)$ - алгебра непрерывных операторов в $E, X$ - компактное топологическое пространство, $A: X \rightarrow \mathcal{L}(E)$ - функция, непрерывная в сильной операторной топологии,

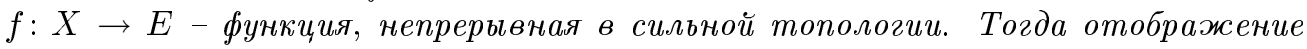
$A f: X \rightarrow E$, определенное формулой $x \mapsto A(x) f(x), x \in X$, сильно непрерьвно на $X$. 
ДокАЗАТЕльство. Множество $A(X)$ поточечно ограничено и поэтому равностепенно непрерьвно [195; III.4.2]. Поэтому достаточно доказать следующую лемму.

ЛЕмма 9. Пусть $E$ - бочечное локально выпуклое пространство, $\mathcal{L}(E)$ - алгебра непрерывных операторов в $E, X$ - топологическое пространство, $A: X \rightarrow$ $\mathcal{L}(E)$ - функиия, непрерывная в сильной операторной топологии и принимающая

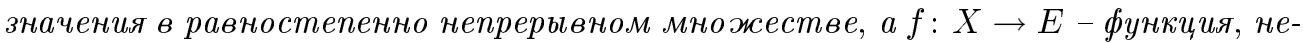
прерывная в сильной топологии. Тогда отображение $A f: X \rightarrow E$, определенное формулой $x \mapsto A(x) f(x), x \in X$, сильно непрерьвно на $X$.

ДокАЗАТЕЛЬство. Пусть $U$ - данная сильная окрестность нуля в $E$ и $V$ - такая окрестность нуля, что $V+V \subset U$. Для окрестности $V$ найдем окрестность нуля $W$ в $E$ так, чтобы $A(X) W \subset V$. Пусть $x_{0} \in X$. Найдем окрестность $O_{1}$ точки $x_{0}$ в $X$ такую, что $f(x)-f\left(x_{0}\right) \in W$ при $x \in O_{1}$, и окрестность $O_{2}$ точки $x_{0}$ в $X$ такую, что $\left(A(x)-A\left(x_{0}\right)\right) f\left(x_{0}\right) \in V$ при $x \in O_{2}$. Тогда при $x \in O \subset O_{1} \cap O_{2}$ получаем

$$
A(x) f(x)-A\left(x_{0}\right) f\left(x_{0}\right)=A(x)\left(f(x)-f\left(x_{0}\right)\right)+\left(A(x)-A\left(x_{0}\right)\right) f\left(x_{0}\right) \in V+V \subset U .
$$

Заметим, что условие локальной равностепенной непрерьвности в сильной операторной топологии автоматически вьполнено для сильно непрерьвных представлений локально компактных групп в бочечных пространствах.

Подготовимся теперь к формулировке критерия бочечности пространства, образованного сильно непрерьвными функциями на компакте со значениями в локально выпуклом пространстве и снабженного равномерной топологией. Обозначим через $M^{\circ}$ поляру подмножества $M$ локально выпуклого пространства $E$, т.е.

$$
M^{\circ}=\left\{f \mid f \in E^{*}, \operatorname{Re}(f(x)) \leqslant 1 \text { для всех } x \in M\right\} .
$$

Напомним следующее определение.

ОПРЕДЕЛЕНИЕ 8. Пусть $E$ - локально вьпуклое пространство. Последовательность $\left\{x_{n} \mid n \in \mathbb{N}\right\}$ называется слабо суммируемой, если $\sum_{n}\left|f\left(x_{n}\right)\right|<\infty$ для каждого непрерьвного линейного функционала $f$ на $E$. Пусть $l^{1}[E]-$ семейство всех таких последовательностей. Для каждой окрестности нуля $U$ в $E$ положим

$$
\varepsilon_{U}\left(\left\{x_{n}\right\}\right)=\sup \left\{\sum_{n}\left|f\left(x_{n}\right)\right| \mid f \in U^{\circ}\right\} \leqslant \rho<\infty .
$$

Локально вьпуклая топология, определяемая семейством преднорм вида $\varepsilon_{U}$, где $U$ пробегает систему окрестностей нуля в $E$, назьвается $\varepsilon$-топологией на $l^{1}[E]$.

Обозначим через $\mathfrak{B}(E)$ семейство всех замкнутых уравновешенных вьпуклых ограниченных подмножеств пространства $E$.

Говорят, что данное локально вьпуклое пространство $E$ имеет свойство (В) Пича (см. $[179 ; 1.5 .5]$ и $[156])$, если для любого ограниченного подмножества $\mathbf{B}$ в $l^{1}[E]$ (в $\varepsilon$-топологии) существует такое множество $B \in \mathfrak{B}(E)$, что $\sum_{n} p_{B}\left(x_{n}\right) \leqslant 1$ для всех $\left\{x_{n}\right\} \in \mathbf{B}$, где $p_{B}(x)=\inf \{\rho>0 \mid x \in \rho B\}-$ функционал Минковского множества $B$.

Напомним, что пространства типа (F) и (DF) обладают свойством (B) (см. [156; 1.5.8]) и сформулируем критерий Мендосы. 
ТеОрема J (критерий Мендосы [156]). Пусть E - бочечное локально выпуклое пространство, $K$ - компакт, $\mathrm{C}(K, E)$ - пространство непрерывных функций на $K$ со значениями в $E$, снабженное топологией равномерной сходимости. Пространство $\mathrm{C}(K, E)$ бочечно тогда и только тогда, когда пространство $E$ бочечно и имеет свойство (В) Пича.

Теперь сфформулируем несколько искусственное утверждение, доказательство которого легко склеивается из готовых частей (т.е. из известных фактов) и хорошо видно, как эти части должны взаимодействовать.

Теорема 6. Пусть Е - бочечное локально выпуклое пространство со свойством Крейна, имеющее также свойство (В) Пича. Пусть WAP ${ }_{s c}(E)$ - пространство, образованное слабо почти периодическими функциями на топологической группе $G$ со значениями в $E$, каждая из которых сильно непрерывна и допускает продолжение на компактную полутопологическую полугруппу $G^{\mathscr{W} \mathscr{P}}$ до функиии, сильно непрерывной на $G^{\mathscr{W} \mathscr{P}}$ (если әта функция рассматривается как отображсене в пространство $E$, снабжсеное сильной топологией).

Пространство $W_{\text {sc }}(E)$ допускает разложение в прямую сумму двух инвариантных замкнутых векторных подпространств, одно из которых образовано почти периодическими функииями на $G$ со значениями в $E$, а другое - всеми сильно равномерно непрерывными на группе $G$ слабо почти периодическими функциями из пространства $W A P_{\text {sc }}(E)$, замкнутая выпуклая оболочка которых содержит функиию, тожсдественно равную нулю.

ДокАЗАТЕльство. Пространство $W A P_{s c}(E)$ естественно изоморфно пространству сильно непрерьвных $E$-значных функций на компакте $G^{\mathscr{W} \mathscr{A} \mathscr{P}}$. Из теоремы J (критерия Мендосы) следует, что пространство $W A P_{s c}(E)$ бочечно. Если пространство $E$ имеет свойство Крейна, то, как легко видеть, пространство $\mathrm{C}\left(G^{\mathscr{W} \mathscr{A} \mathscr{P}}, E\right)$, снабженное равномерной топологией, также имеет свойство Крейна, а тем самым и его замкнутое подпространство $W A P_{s c}(E)$ имеет свойство Крейна. Поэтому утверждение непосредственно следует из теоремы 1.

Вернемся к спектральньм условиям почти периодичности, которые обсуждались в предыдушем пункте.

Теорема 7. Пусть $G$ - коммутативная топологическая группа, $E$ - пространство Фреше, $f$ - слабо почти периодическая функция на $G$ со значениями в Е. Если спектр функиии $f$ дискретен, то функция $f$ почти периодична.

ДоКАЗАТЕЛЬСТВо. Из условия следует почти периодичность матричных элементов функции $f$ (т.е. функций $\varphi \circ f, \varphi \in E^{*}$ ). Остается применить приведенную вьше теорему 5 .

Желательно распространить утверждение на случай некоммутативных групп.

5.5. Почти периодичность функций с почти периодическими разностями и почти периодичность неопределенного интеграла.

Одна из самых старых задач теории почти периодических функций - задача об условиях почти периодичности неопределенного интеграла от почти периодической функции. Для условно-периодических функций она была решена еше Болем [45]. И для скалярных функций, и для векторных функщий задача о неопределенном интеграле 
сводится к задаче о разностях [12]. Приведем формулировку утверждения, которое является непосредственньм следствием теоремы 5 .

ТеОРема 8. Пусть $f$ - слабо почти периодическая функиия на топологической группе $G$ со значениями в пространстве Фреше $E$. Функиия $f$ почти периодична тогда и только тогда, когда все матричнье әлементы функции $f$ почти периодичны (т.е. все функиии $\varphi \circ f, \varphi \in E^{*}$, являются комплексными почти периодическими функииями на $G)$.

ДокАЗАТЕльство. Применим к функции $f$ разложение, приведенное в теореме 5. Из условия о матричных элементах следует, что диссипативная компонента вектора $f$ равна нулю, и поэтому этот вектор (функция $f$ ) принадлежит подпространству аппроксимативно конечномерных векторов.

Другое доказательство может быть основано на критерии компактности в терминах крайних точек [49].

Сформулируем теперь теорему о разностях.

ТеОрема 9. Пусть $f$ - слабо почти периодическая функция на топологической группе $G$ со значениями в пространстве Фреше E. Если все разности вида $g \mapsto$ $f(g h)-f(g), g \in G$, являются почти периодическими функциями, то функция $f$ почти периодична.

ДокАЗАТЕльство. Из предыдушей теоремы следует, что достаточно доказать теорему для матричных элементов $\varphi \circ f, \varphi \in E^{*}$. Но к этим матричным элементам можно применить теорему о разностях для числовых функций.

\section{§ 6. Примеры}

Первьй из примеров носит фольклорньй характер. Даже в этой статье мы встречаем его не в первьй раз: он использовался в $\S 4$ в другом контексте.

ПримеР 1. Пример функции с разреженным спектром, не являюшейся почти периодической. Пусть $f$ - равномерно непрерьвная ограниченная функция на $\mathbb{R}$ со значениями в пространстве $c$ сходящихся комплексных последовательностей, определенная формулой $f(t)=\{\exp (t / n) \mid n \in \mathbb{N}\}$. Тогда спектр функции $f$ счетен $(\operatorname{sp} f=\{1 / n \mid$ $n \in \mathbb{N}\} \cup\{0\}$ ) и поэтому разрежен, а функщия скалярно почти периодична (потому что сопряженное пространство образовано сходящимися рядами), но не почти периодична (см. $\S 4)$. Функция $f$ является неопределенньм интегралом от почти периодической функции $f^{\prime}(t)=\{(1 / n) \exp (t / n) \mid n \in \mathbb{N}\}$.

Напомним, что $f$ не является и слабо почти периодической функцией, см. $\S 4$.

Следующие примеры принадлежат Гюнщлеру [104].

ПРимеР 2. Пример скалярно почти периодической функции, слабый спектр которой несчетен. Пусть $G=\mathbb{R}_{d}$ (аддитивная группа вешественных чисел в дискретной топологии). Пусть $\Omega$ - базис Гамеля (базис в $\mathbb{R}$, рассматриваемом как линейное пространство над полем $\mathbb{Q}$ рациональных чисел). Можно считать, что $2 \pi \in \Omega$. Положим

$$
f(s)(\omega)=\varphi\left(r_{\omega}(s)\right)-1, \quad s \in \mathbb{R}_{d}, \quad \omega \in \Omega,
$$


где величины $r_{\omega}, \omega \in \Omega$, определены формулой

$$
x=\sum_{\omega \in \Omega} r_{\omega}(x) \omega, \quad x \in \mathbb{R},
$$

и $\varphi(t)=e^{i \pi t}$ при $t \in \mathbb{R}$. Как и в предыдущем примере, функция $f$ скалярно почти периодична, но ее слабьй спектр равен

$$
\bigcup_{\omega \in \Omega} \sigma\left(\varphi \circ r_{\omega}-1\right)=\bigcup_{\omega \in \Omega}\left\{\{1\},\left\{\varphi \circ r_{\omega}\right\}\right\}=\{\{1\}\} \cup\left\{\left\{\varphi \circ r_{\omega}\right\} \mid \omega \in \Omega\right\}
$$

и множество $\Omega$ несчетно вместе с $\mathbb{R}$.

ПримеР 3. Пример функшии с одноточечным спектром, разрывной в обычной топологии числовой прямой. Любая функщия вида $f=\varphi \circ r_{\omega}$ (см. предыдуший пример) скалярно почти периодична, но не является непрерывной на $\mathbb{R}$ в обычной топологии прямой.

\section{§7. Другие результаты, связанные с классическими задачами теории почти периодических функций}

\section{1. Непосредственные следствия теоремы разложения.}

“Теорема разложения", распространяющая результаты де Лю и Гликсберга на случай представлений в бочечных локально выпуклых пространствах со свойством Крейна, имеет ряд других непосредственных следствий. Приведем обобшения двух утверждений де Лю и Гликсберга.

С ЛЕДСТВИЕ 1. Пусть Е - бочечное локально выпуклое пространство со свойством Крейна, $\mathscr{L}(E)$ - алгебра всех непрерывных линейных операторов в $E$ и $S$ - подмножество $\mathscr{L}(E)$. Если $S$ компактно в слабой операторной топологии иявляется группой относительно обычного умножсения операторов, и если е единичный әлемент группы $S$, то е-непрерывный проектор в $E, E_{v}=\operatorname{Ker} e$, $E_{r}=\operatorname{Im} e$, и группа $S$ компактна в сильной операторной топологии.

СлЕДСТВИЕ 2. Пусть Е - бочечное локально выпуклое пространство со свойством Крейна, $\mathscr{L}(E)$ - алгебра всех непрерывных линейных операторов в $E$ и $S$ - подмножсество $\mathscr{L}(E)$. Если $S$ компактно в слабой операторной топологии, замкнуто относительно обьчного умножения операторов и состоит из обратимых операторов, то $S$ - группа, компактная в сильной операторной топологии, причем $E=E_{r}$.

Доказательства этих следствий повторяют доказательства теоремы 8.1 и следствия 8.2 в [67] с минимальньми изменениями. 
7.2. Дополнительные сведения о почти периодических функциях и представлениях.

Следуя комбинаторной идее А. А. Маркова, примененной им в 1933 г. в доказательстве связи между условиями устойчивости и почти периодичности решений некоторых диффреренциальных уравнений [150], Финк и Майстерс [85] назьвают подмножество $S$ в групп $G$ подмножсеством типа $(\Delta-m)$ (или, коротко, $(\Delta-m)$-подмножсеством), если $S=S^{-1}$ и для любых $t_{1}, \ldots, t_{m+1} \in G$ сушествуют такие индексы $i \neq j$, что $t_{i}-t_{j} \in S$. В [85] доказано, что функция $f$ почти периодична по фон Нейману тогда и только тогда, когда множество ее $\varepsilon$-почти периодов есть $(\Delta-m)$-множество.

В [127] рассматривается ряд обобщений понятия почти периодической функции на полутопологической полугруппе $S$ с единичным элементом и доказьвается, что некоторые из этих определений равносильны обычной почти периодичности, что приводит к новым критериям почти периодичности. Например, непрерывная функция $f$ на $S$ называется удовлетворяющей условию (В) относительно класса $\mathscr{A}$ подмножеств полугрупшы $S$, если для любого $\varepsilon>0$ существует такое $A \in \mathscr{A}$, что множество $\{\sigma \in A|| f(x y z)-f(x \sigma z) \mid<\varepsilon$ для всех $x, z \in S\}$ непусто для всех $y \in S$. Очевидно, что если семейство $\mathscr{A}$ состоит из конечных множеств, то $f$ почти периодична. Другие критерии используют предположение, что $\mathscr{A}$ состоит из вполне ограниченных слева подмножеств, а $f$ равномерно непрерьвна справа.

Функция $f \in \mathrm{CB}(S)$ назьвается равномерно непрерьвной справа (слева) в точке $s \in S$, если $\left\|L_{s_{\alpha}} f-L_{s} f\right\| \rightarrow 0\left(\left\|R_{s_{\alpha}} f-R_{s} f\right\| \rightarrow 0\right)$ для любой сети $s_{\alpha}$ в $S$, сходящейся к $s$ в $S$. В [158] получен ряд характеризаций почти периодических функций (в том числе в терминах правой и левой равномерной непрерьвности) в некоторых классах обобщенных почти периодических функций (в том числе введенных в [127]). Кроме того, в [158] показано, что любая функция из $\mathrm{C}(S)$, где $S$ - компактная полутопологическая полугруппа, равномерно непрерьвна справа и слева в единичном элементе, и $S$ является топологической полугруппой (т.е. умножение в $S$ непрерывно по совокупности переменных) тогда и только тогда, когда каждая функция из $\mathrm{C}(S)$ равномерно непрерьвна справа и слева в каждой точке полугрупшы $S$.

В [157] сравниваются три пространства, связанные с векторнозначными слабо почти периодическими функциями. Пусть $\mathrm{CB}(S, X)$ - банахово пространство ограниченных непрерьвных функций на полутопологической полугрупе $S$ со значениями в банаховом пространстве $X$. Пусть $W R(S, X)$ состоит из всех таких функций $f \in$ $\mathrm{CB}(S, X)$, у которых правая орбита $R_{S} f=\left\{R_{s} f \mid s \in S\right\}$ относительно слабо компактна в $\mathrm{CB}(S, X)$. Пусть пространство $W R C(S, X)$ состоит из всех $f \in W R(S, X), \mathrm{y}$ которых область значений $f(S)=\{f(s): s \in S\}$ вполне ограничена, а пространство $W(S, X)$ состоит из всех функций $f \in \mathrm{CB}(S, X)$, удовлетворяющих условию, что все функции $s \rightarrow x^{*}(f(s))$ являются обычными числовыми слабо почти периодическими функциями на $S$ при любых $x^{*} \in X^{*}$. Используя свое новое обобшение критерия Гротендика для слабой компактности [100], автор статьи [157] показывает на примерах, что все эти три пространства могут попарно не совпадать.

7.3. Свойства почти периодических функций: существование среднего значения, спектр, теоремы о разностях и об интеграле, условия компактности, теорема Дини, свойства диссипативных функций.

В [40] существование инвариантного среднего на почти периодических функциях Бохнера на абелевой групе $G$ со значениями в $\mathbb{C}^{n}$ доказывается с помошю следую- 
щей теоремы о неподвижной точке. Пусть $(X,|\cdot|)$ - комплексное банахово пространство, $(\cdot, \cdot)$ - непрерывное по норме $|\cdot|$ скалярное произведение на $X$ с нормой $\|\cdot\|, G-$ абелева полугруппа $|\cdot|$-непрерывных линейных $\|\cdot\|$-изометрий с $|g| \leqslant 1$ при $g \in G$, а $z \in X$ - такой элемент, что множество $Z=\{g z \mid g \in G\}$ относительно $|\cdot|$-компактно. Пусть $K-|\cdot|$-замкнутая выпуклая оболочка множества $Z$. При этих условиях существует в точности один такой элемент $y \in K$, что $g y=y$ при $g \in G$, и $\{u \in K \mid g u=u\}$ - замкнутая вьпуклая оболочка множества $\left\{h z^{g} \mid h \in G\right\}, g \in G$; здесь $x^{g}$ определяется как предел по норме $|\cdot|$ последовательности

$$
(1 / n) \sum_{0}^{n-1} g^{k} x
$$

который существует при $x \in K, g \in G$, и отображение $x \mapsto x^{g}$ вьпукло на $K$, причем $g x^{g}=x^{g}$ и $x-x^{g} \perp$ inv $g$. (Доказательство этого факта использует теорему Маркова-Какутани и интеграл по мере Хаара на компактификации Бора в качестве среднего и поэтому вьглядит крайне несовременным по сравнению с доказательствами Маака и особенно Гринлифа, доказьвающего существование среднего на не обязательно коммутативных группах с помощью теоремы Рылль-Нардзевского о неподвижной точке [191].) Тем не менее формулировка утверждения наглядна, и поэтому интересно, можно ли распространить эту теорему Бло о неподвижной точке хотя бы на случай аменабельных полугрупп изометрий.

В [6] обсуждаются различные определения спектра и схемы доказательства теоремы об интеграле (с помощью теоремы о разностях) и некоторые варианты и обобщения теоремы Люмиса о разреженном спектре (см. [141; гл. VI]), а также доказательство теоремы, утверждающей, что ограниченная равномерно непрерьвная функция на коммутативной локально компактной групе почти периодична, если спектр Арвесона (см. [8]) этой функции дискретен. Этот подход существенно развивается в [16], [17], [22] с помощью общего понятия спектра элемента относительно класса функций. Пусть $\mathrm{UCB}(\mathbf{J}, X)$ - банахово пространство всех равномерно непрерьвных ограниченных функций на направленном вправо луче или на всей прямой $\mathbf{J}\left(\mathbf{J} \cap \mathbb{R}_{+} \in\left\{\mathbf{J}, \mathbb{R}_{+}\right\}\right.$ или $\mathbf{J}=\mathbb{R})$ со значениями в банаховом пространстве $X$. Пусть $\mathscr{F}-$ класс функций в пространстве $\mathrm{UCB}(\mathbf{J}, X)$. Понятие спектра $\operatorname{sp}_{\mathscr{F}}(\varphi)$ функции $\varphi \in \operatorname{UCB}(\mathbb{R}, X)$ относительно класса $\mathscr{F}$ определяется как множество общих нулей преобразований Фурье суммируемых функций, ограничение свертки которых с данной функцией на луч (если это луч, а не прямая) принадлежит классу $\mathscr{F}$. С помощью этого понятия спектра исследуются задачи о разностях и интеграле. Сходные результаты получил ранее Баскаков для $\mathbf{J}=\mathbb{R}[27]$. Несколько более слабые утверждения приведены в [105].

В [33] приведен краткий обзор известных в то время свойств так назьваемых диссипативных слабо почти периодических функций (т.е. функций, среднее значение модуля которых равно нулю). В частности, приведен пример Рудина диссипативной функции на групше целых чисел, не допускающей равномерной аппроксимации преобразованиями Фурье-Стилтьеса элементов алгебры мер на окружности. Аналогичный пример приведен в [34], где также доказаны следуюшие утверждения.

ТЕОРемА K [34]. Пусть $G$ - локально компактная топологическая группа, $S$ - ее замкнутая некомпактная подполугруппа. Пусть пространство WAP $(S)$ 
имеет инвариантное среднее, а групповая операция непрерывна в каждой точке произведения $S \times S^{w}$, где $S^{w}$ - слабо почти периодическая компактификация nолугруппь $S$.

(I) Пусть $f \in W A P(S)$. Следующие условия равносильны:

(1) каноническое непрерывное продолжение $f^{w}$ функиии $f$ на $S^{w}$ обращается в нуль на минимальном идеале (ядре) $K\left(S^{w}\right)$ полугруппь $S^{w}$;

(2) $M(|f|)=0$, где $M$ - единственное инвариантное среднее на $W A P(S)$;

(3) нулевая функиия принадлежит слабому замыканию орбиты функиии $f$ в $W A P(S)$

(4) для любого $\varepsilon>0$ и любого компактного подмножества $K$ в $S$ существует такой элемент $s \in S$, что $\varepsilon>\sup \{|f(k s)| \mid k \in K\}$.

(II) Пусть, кроме того, подполугруппа $S$ содерэит единичный әлемент группы $G$, а функция $f \in W A P(G)$ диссипативна (т.е. удовлетворяет әквивалентнымм условиям (1)-(4)). Пусть $U_{0}$ - компактная окрестность единичного әлемента в $G$, и пусть $\varepsilon>0$. Тогда существует такая компактная окрестность $V=V\left(U_{0}, f, \varepsilon\right)$ единичного әлемента в $G$, что для любого $s \in S$ существует әлемент $r \in S$, удовлетворяющий условиям $(V+s) \cap\left(U_{0} \cap S+r\right) \neq \varnothing и$ $\varepsilon>\sup \left\{|f(t r)| \mid t \in U_{0} \cap S\right\}$.

Пусть $G$ - бесконечная дискретная группа. Связь теории слабо почти периодических функций с теорией так назьваемых тонких множеств в $G$ обсуждалась в [95], где рассматривались продолжения ограниченных непрерьвных функций на подмножествах до почти периодических функций на группе, и в [57], где изучались связи между множествами Сидона в $G$, такими множествами $F \subset G$, что все пересечения вида $F \cap g F$ и $F \cap F g, g \neq e$, конечны, и такими множествами $F \subset G$, что любая функция на $G$, обрашающаяся в нуль вне $F$, слабо почти периодична как элемент пространства $l^{\infty}(G)$. Следуюшие важные количественные результаты об аддитивной полугруппе $\mathbb{N}$ неотрицательных целых чисел были получены в этом направлении в $[185]$. Пусть $W(\mathbb{N})$ - пространство слабо почти периодических функций на $\mathbb{N}$, $W_{0}(\mathbb{N})=\{f \in W(\mathbb{N}) \mid$ функция $|f|$ имеет нулевое среднее $\}$ - подпространство диссипативных функций. Пусть

$$
W_{00}(\mathbb{N})=\left\{f \in W_{0}(\mathbb{N}) \mid \text { если } k_{n} \rightarrow \infty \text { и } f_{k_{n}} \rightarrow g \text { слабо, то } g \rightarrow C_{0}(\mathbb{N})\right\},
$$

где $f_{k}$ обозначает сдвиг $f$ на $k$ при $k \in \mathbb{N}$. Напомним, что множество Сидона в $\mathbb{N}-$ это такое множество $E \subset \mathbb{N}$, что любой элемент пространства $c_{0}(E)$ последовательностей на $E$, стремящихся к нулю на бесконечности, есть ограничение на $E$ последовательности коэффициентов Фурье суммируемой функции. Оказьвается, если $A-$ множество Сидона, то его характеристическая функция принадлежит пространству $W_{00}(\mathbb{N})$. На примерах показано, что $W_{00}(\mathbb{N})$ - правильная часть пространства $W_{0}(\mathbb{N}) ;$ более того, существует такая функция $f \in W_{0}(\mathbb{N}) \backslash C_{0}(\mathbb{N})$, что $\left\|f_{k_{n}}-f\right\|_{\infty} \rightarrow 0$ по некоторой последовательности $k_{n} \rightarrow \infty$.

И условия сильной компактности, и условия слабой компактности подмножеств пространств почти периодических или слабо почти периодических функций являются частными случаями вопросов о компактности и слабой компактности подмножеств в пространствах ограниченных функций или (сильно или слабо) непрерьвных функций и поэтому непосредственно связаны с общими задачами линейного анализа (см. [76], 
[128], [129], [208]), но иногда бывает нужно располагать условиями компактности в тех или иных специальных терминах. В [29] приведен ряд условий того, что данная ограниченная последовательность почти периодических функций на числовой прямой слабо сходится. Эти условия позволяют охарактеризовать относительно слабо компактные подмножества в пространстве почти периодических функций на прямой. Распространение этих результатов на другие групшы и на случай функций с векторными значениями представляет нерешенную задачу.

Родственной проблемой является аналог теоремы Дини для почти периодических функций. Пусть $G$ - группа. Функщия $f$ назьвается почти периодической по Мааку, если для любого $\varepsilon>0$ сушествует конечное покрытие $G=\bigcup_{i} A_{i}, 1 \leqslant i \leqslant n$, удовлетворяющее условию $|f(x a y)-f(x b y)|<\varepsilon$ для всех $x, y \in G$ и всех $a, b \in A_{i}$, $1 \leqslant i \leqslant n$. Согласно [98], если монотонно неубьвающая последовательность (вещественных) почти периодических функций на группе $G$ сходится поточечно к почти периодической функции, то эта последовательность сходится равномерно. Было бы интересно выяснить возможности распространения этой теоремы на другие классы почти периодических функций и прежде всего - на слабо почти периодические функции.

Область приложения теории почти периодических функций к дифференциальньм уравнениям настолько обширна, что даже ссылок на указанные выше обзоры по частным вопросам [130], [196], [205] недостаточно, чтобы составить представление о богатстве этой тематики. Мы избегаем цитирования работ по теории дифференциальных уравнений, даже если они существенно используют аппарат почти периодических функций, но обсудим вопросы, важные для самой теории почти периодических функций и ее приложений в других областях. Так, вопрос о существовании почти периодического решения обыкновенного дифференциального уравнения с постоянными коэффициентами с почти периодической правой частью сводится к вопросу о скалярной почти периодичности неопределенного интеграла от скалярно почти периодической функции. Это - одна из центральных задач в книге Америо [2], где подробно рассматриваются почти периодические функции и скалярно почти периодические функции (последние - под названием “слабых" почти периодических функций, как и в книге [141]) и их приложения к теории дифференциальных уравнений. В частности, в [2] получено следуюшее обобшение теоремы Боля-Бора: если $J$ - бесконечньй или полубесконечнцй промежуток на $\mathbb{R}$ и если $f: J \rightarrow X$ - почти периодическая функция со значениями в равномерно выпуклом банаховом пространстве $X$ с ограниченньм неопределенным интегралом, то этот интеграл - почти периодическая функция. Полезное упражнение - вывести эту теорему при $J=\mathbb{R}$ из теоремы 1 . Указание состоит в автоматической рефлексивности всех равномерно вьпуклых банаховых пространств [66] и слабой компактности ограниченных подмножеств рефилексивных пространств.

Описанный вьше спектральньй подход работы [6] к теореме Кадеца об интеграле и о разностях и к теореме о почти периодичности ограниченной равномерно непрерьвной функции на коммутативной локально компактной группе (в предположении, что спектр Арвесона [8] этой функции дискретен) обобщался в другом направлении в [12] и в [15], где рассматривался неопределенный интеграл от функции со значениями в полном локально выпуклом топологическом векторном пространстве, изучались его общие свойства и рассматривались интегралы от функций со значениями в равномерно замкнутом инвариантном относительно сдвигов подпространстве пространства рав- 
номерно непрерьвных функций. В [18] был получен вариант теоремы о разностях для функций на топологической группе со значениями в пространстве $\Phi$ реше $A$, связанный со следуюшей теоремой непрерьвности. Пусть $\gamma \in G$ и функция $\Delta_{\gamma} f$ определена формулой $\left(\Delta_{\gamma} f\right)(t)=f(t \gamma)-f(t), t \in G$. Пусть $\Delta_{\gamma} f$ непрерьвна в единичном элементе $e$ группы $G$ для всех $\gamma$ из некоторой окрестности элемента $e$. Тогда $f$ непрерывна в $e$, если $A$ не содержит подпространства, изоморфного $c_{0}$, а $f$ ограничена. Аналогичншй результат получен, если $A$ - локально выпуклое пространство и область значений $f$ слабо относительно компактна. Сходные условия непрерьвности были использованы в [158] в доказательстве почти периодичности функций (в духе [127]) при следующих предположениях. Пусть $S$ - полутопологическая полугруппа с единичным элементом $e, f$ - такая ограниченная непрерывная функция на $S$, что для любого $\varepsilon>0$ найдется такое вполне ограниченное справа множество $A$ (т.е. для любой окрестности $V$ единичного элемента $e$ существует такое натуральное число $N$ и такие элементы $t_{1}, \ldots, t_{n} \in A$, что $\left.A \subset \bigcup\left\{V t_{i} \mid 1 \leqslant i \leqslant n\right\}\right)$, что множество $\left\{s \in A \mid\left\|L_{s} f-L_{t} f\right\|<\varepsilon\right\}$ непусто для всех $t \in S$. В [158] доказано, что если $\left\|L_{s_{\alpha}} f-f\right\| \rightarrow 0$ для любой сети $s_{\alpha}$ в $S$, сходящейся в $S$ к $e$, то $f$ - почти периодическая функция на $S$.

Хотя линейные дифференциальные уравнения высших порядков с почти периодическими коэффициентами могут не иметь почти периодических решений, некоторые задачи о линейных дифференциальных уравнениях с почти периодическими коэффициентами имеют большое значение и в самой теории, и в ее приложениях. Полугруппы операторов и периодические эволюционные системы образуют пограничную область между ядром теории и ее приложениями к топологической динамике и дифференциальным уравнениям. Результаты работы [188] особенно близки одной из основных тем настоящего обзора: там уточняются условия на банахово пространство $X$ и асимптотическую траекторию $u:[0, \infty) \rightarrow X$ периодической эволюционной системы, обеспечиваюшие слабую почти периодичность траектории $u$. Этот результат, вместе с теорией де Лю-Гликсберга [67] почти периодических функций на полугруппах, позволяет распространить эргодические теоремы о сильных пределах на случай обших периодических эволюционных систем. Получены также условия слабой почти периодичности решений неавтономных (линейных и нелинейных) задач Коши и о существовании почти периодических и периодических решений таких задач. Как показывают примеры, условие периодичности системы здесь нельзя заменить условием почти периодичности. В связи с этим замечателен результат статьи [169], из которого следует, что все траектории неавтономного уравнения $\dot{X}=A(t) X$, где $X \in S O(n)$ и $A(t) \in s o(n)$ - почти периодическая функция со значениями в кососимметрических матрицах, бесконечно много раз возвращаются к начальному условию с заданной точностью (для условно периодического коэффициента есть количественная версия этого утверждения). Аналогичный результат справедлив на любой простой компактной связной группе Ли.

В применении к решениям дифференциальных уравнений, связь между компактностью множества значений решения абстрактного дифференциального уравнения с оператором, порождаюшим сильно непрерьвную однопараметрическую группу ограниченных операторов в банаховом пространстве, и почти периодичностью этого решения была установлена уже в [235]. Условия принадлежности высших производных решения линейной дифференщиально-разностной системы на прямой или полупрямой тому или иному классу функций со значениями в банаховом пространстве (в том чис- 
ле классам почти периодических, асимптотически почти периодических, слабо почти периодических и равномерно непрерьвных функций и различным классам эргодических функций) в связи с некоторыми наследственньми свойствами классов почти периодических функций и их обобшений получены в [20]. Принадлежность решений полулинейных абстрактных дифференциальных уравнений $u^{\prime}(t)=A u(t)+f(t, u(t))$ различньм классам почти периодических функций, где $A$ - порождающий оператор $C_{0}$-полугруппы, и связанные с этим свойства почти периодических функций исследовались в большом числе работ. Современная библиография по этой теме приведена В $[24]$.

\section{4. Обобщения почти периодических функций и топологическая ди-} намика.

Обобщения понятия почти периодической функции весьма многообразны. Это, в частности, асимптотически почти периодические функции, асимптотически слабо почти периодические функции, псевдо-почти периодические функции (см., например, [231], [232]), почти периодические функции Левитана (см., например, [11], [13], [14], [141]), равносильные почти автоморфным функциям Бохнера [41]-[43] (см. также [215]; так назьваемьм возмущениям почти периодических функций Левитана и соответствующей теореме о неопределенном интеграле посвящена работа [21]), дистальные (мы кратко обсуждаем их ниже), рекуррентные (которые, оказывается, нужно отличать от эргодических [1]) и многие другие. В [176] класс почти периодических функций расширяется с помошью интегрирования по Данжуа.

Асимптотически почти периодические функции на правом луче $\mathbf{J} \subset \mathbb{R}$ могут быть определены несколькими способами: с помошью понятия почти периода, с помошью условия компактности (или слабой компактности) орбиты, отвечающей лучу, и как семейство сумм почти периодических (слабо почти периодических) функций и функций, стремяшихся к нулю на бесконечности (ср. [22], [187]). Один из самых замечательных результатов статьи [187] состоит в том, что если $X$ - банахово пространство, $a \in \mathbb{R}, \mathbf{J}=(a,+\infty), f$ - асимптотически почти периодическая функция на $\mathbf{J}$ со значениями в $X$, а $F: \mathbf{J} \rightarrow X$ определена формулой

$$
F(t)=\int_{a}^{t} f(u) d u, \quad t \in \mathbf{J}
$$

и если либо образ функции $F$ на $\mathbf{J}$ ограничен в $X$ и $X$ не содержит изоморфной копии пространства $c_{0}$, либо образ функции $F$ на $\mathbf{J}$ слабо относительно компактен в $X$, то $F$ является асимптотически почти периодической функцией тогда и только тогда, когда $f$ слабо почти периодична, а также тогда и только тогда, когда стремящееся к нулю слагаемое несобственно интегрируемо по Риману на $\mathbf{J}$. Аналогичньй результат о слабой почти периодичности траекторий те же авторы получают в [188] для нелинейных полугрупп преобразований. В [52] указаны условия асимптотической почти периодичности функции $f:[0, \infty) \rightarrow X$, где $X$ - банахово пространство, в терминах поведения преобразования Лапласа $\widehat{f}$ функции $f$ вблизи мнимой оси. В частности, если $f$ ограничена и удовлетворяет некоторому условию типа эргодичности, а функция $\widehat{f}$ имеет лишш счетное множество особенностей на мнимой оси, то $f$ асимптотически почти периодична. Этот результат связан с изучением асимптотического поведения и устойчивости $C_{0}$-полугрупш в банаховых пространствах, см. [5], [10], [148], [218]. 
Пусть $X$ - банахово пространство, $\mathbf{J} \in\left\{\mathbb{R}^{+}, \mathbb{R}\right\}$ и $\mathrm{CB}(\mathbf{J}, X)$ - банахово пространство ограниченных непрерьвных функций на $\mathbf{J}$ со значениями в $X$. В [26] введены классы функций в $\mathrm{CB}(\mathbf{J}, X)$, содержашие некоторые известные классы функций типа почти периодических. В [25] изучаются эргодические функции в смысле Изеки, которьй ввел в [114] общее понятие эргодичности функции на абелевой полугруппе как условие, что средние арифметические некоторых сдвигов этой функции равномерно приближают некоторую постоянную. Это понятие позволяет расширить область применимости подхода Эберлейна [74] к эргодичности полугрупп операторов в $X$. Для инвариантных относительно сдвигов замкнутых подпространств $\mathscr{A}$ пространства всех ограниченных функций на $J$ со значениями в $X$ получена теорема о разностях (показано, что если $\mathscr{A}$ содержит постоянные функции и $\phi$ - эргодическая функция, разности которой лежат в $\mathscr{A}$, то $\phi \in \mathscr{A}$ ), положившая основу исследований в [16], [17], [22], результаты которых описаны выше в п. 7.3. Для псевдо-почти периодических функций вопросы интегрирования рассматривались в [231], [232]. В [23] понятие эргодичности распространено на случай неограниченных функций $\varphi: J \rightarrow X$, где $J$ - подполугруппа локально компактной абелевой группы $G$ и $X$ - банахово пространство, а функции $\varphi$ предполагаются непрерьвньми и мажорируемыми некоторым весом $w$ на групе $G$. В частности, установлена полная эргодичность орбит (неограниченных) сильно непрерьвных представлений $T: G \rightarrow L(X)$, дуальные представления которых не имеют точечного унитарного спектра. Изучение спектров функций использует технику алгебр Берлинга $L_{w}^{1}(G)$, для которых получено новое описание пространств максимальных примарных идеалов для неквазианалитических весов $w$. В терминах транслящионно инвариантных классов функций получены условия непустоты приведенного спектра Берлинга, обобщающие тауберову теорему Винера.

Во многих случаях теорема 1 допускает распространение на более широкий класс представлений групп без изменения технических приемов за счет модификации топологии в функциональном пространстве. Например, пусть $G$ - локально компактная хаусдорфова группа, снабженная левой мерой Хаара. Если $f \in L^{1}(G)$, то отображение $g \mapsto f * g, g \in L^{\infty}(G)$, задает линейное преобразование пространства $L^{\infty}(G)$. Обозначим через $\tau_{c}\left[\tau_{w}\right]$ слабейшую топологию на $L^{\infty}(G)$, в которой все эти преобразования становятся непрерывными отображениями в $\left(L^{\infty}(G),\|\cdot\|_{\infty}\right)\left[\right.$ в $\left(L^{\infty}(G), \sigma\right)$, где $\sigma$ означает слабую топологию на $\left.L^{\infty}(G)\right]$. В $[63]$ введено множество $R$ - $\tau_{c}$ - $\mathrm{AP}\left[R-\tau_{w}\right.$-AР $]$ функций из пространства $L^{\infty}(G)$, которые почти периодичны справа относительно $\tau_{c}$ [относительно $\tau_{w}$ ] как функщии, правые орбиты которых относительно $\tau_{c}$-компактны $\left[\tau_{w}\right.$-компактны $]$. Показано, что множество $R$ - $\tau_{c}$-АР является $\tau_{c}$-замыканием множества $A P(G)$, а $R$ - $\tau_{w}$-АР является $\tau_{c}$-замьканием множества $W A P(G)$. (Если $G$ бесконечна, то эти замыкания строго содержат $A P(G)$ и $W A P(G)$ соответственно.) Так как левый сдвиг $\tau_{c}$-непрерьвен, можно продолжить единственное (лево-) инвариантное относительно сдвигов среднее на $A P(G)$ до аналогичного среднего на $R$ - $\tau_{c}$-АР. Полученное продолжение является топологически (лево-) инвариантным средним и оно единственно. Векторные аналоги этих классов почти периодических функций и слабо почти периодических функций вьплядят естественной областью для проверки аналога теоремы разложения.

Есть и другие классы функщий, обобщающие стандартные почти периодические функции и доказавшие свою продуктивность в гармоническом анализе, как почти периодические функции Бора-Френеля [175] и их обобшения на высшие порядки и про- 
извольные локально компактные абелевы группы [199]. Если рассматриваемая полугруппа является аменабельной групой, то “равномерное” условие эргодичности по Изеки означает, что все инвариантные средние (неотрищательные непрерьвные функционалы на пространстве ограниченных непрерывных функций на полугруппе, принимаюшие единичное значение на единичной функции и инвариантные относительно сдвигов на полугруппе) принимают на этой функции одно и то же значение (см., например, [201]). В 1978 г. Жиков [237] (см. также более подробное изложение в [236]) ввел эргодические алгебры в $\mathbb{R}^{n}$, используя неравномерньй вариант определения среднего, применявшийся ранее Безиковичем [38] при введении его класса почти периодических функций. Опишем вариант конструкции Жикова в несколько более общей ситуации, а именно для сепарабельных аменабельных локально компактных групп.

ОПРЕДЕЛЕниЕ 9 . Пусть $G$ - локально компактная группа с левоинвариантной мерой Хаара $\mu, K$ - компактное подмножество в $G$, а $\delta>0$ - число. Компактное множество $F \subset G$ назьвается $(K, \delta)$-инвариантным, если $\mu(F \Delta K F)<\delta \mu(F)$. Последовательность $F_{n}, n \in \mathbb{N}$, компактных подмножеств в $G$ назьвается последовательностью Фолнера, если для любого компакта $K$ в $G$ и любого $\delta>0$ множество $F_{n}$ является $(K, \delta)$-инвариантным при достаточно больших $n$. Последовательность $\left\{K_{n}\right\}$, $n \in \mathbb{N}$, симметричных $\left(\left(K_{n}\right)^{-1}=K_{n}\right)$ компактных подмножеств положительной меры в группе $G$, удовлетворяюших следующим условиям:

(1) последовательность $K_{n}$ монотонно возрастает по включению, т.е. $K_{n} \subset K_{m}$ при $n<m$,

(2) последовательность $K_{n}-$ исчерпывающая, т.е. $G=\bigcup\left\{K_{n} \mid n \in \mathbb{N}\right\}$,

(3) предельное соотношение $\mu\left(g K_{n} \cap K_{n}\right) / \mu\left(K_{n}\right) \rightarrow 0$ вьполняется равномерно на компактах,

называется суммирующей последовательностью [177].

Локально компактная група $G$ с левоинвариантной мерой Хаара $\mu$ назьвается аменабельной, если пространство $L^{\infty}(G)$ существенно ограниченных измеримых функций на $G$ допускает (лево)инвариантное среднее. Оказьвается (см., например, $[177$; утверждения 4.5-4.16 и упражнение 1-10]), для сепарабельной локально компактной группы $G$ следуюшие условия равносильны:

(1) группа $G$ аменабельна,

(2) групша $G$ допускает последовательность Фолнера $F_{n}, n \in \mathbb{N}$, удовлетворяющую условию $\bigcup_{n} F_{n}=G$,

(3) группа $G$ допускает суммирующую последовательность.

Кроме того, каждая монотонно возрастающая (по включению) исчерпьвающая последовательность Фолнера является суммируюшей последовательностью.

Предположим, что задано биизмеримое действие групшы $G$ слева сохраняющими меру преобразованиями на лебеговском вероятностном пространстве $(X, \mathscr{A}, \nu)$ с $\nu(X)=1$. Для функции $f: X \rightarrow \mathbb{R}$ обозначим через $A(F, f)(x)$ среднее

$$
A(F, f)(x)=\mu(F)^{-1} \int_{F} f(g x) d \mu(g) .
$$


ОПРЕДЕЛЕНИЕ 10 . Последовательность Фолнера $F_{n}$ называется умеренной, если существует такая постоянная $C>0$, что

$$
\mu\left(\bigcup_{k<n} F_{k}^{-1} F_{n}\right) \leqslant C \mu\left(F_{n}\right)
$$

для всех $n \in \mathbb{N}$.

Справедлива следующая эргодическая теорема.

Tеорема L (индивидуальная эргодическая теорема для сепарабельных аменабельных локально компактных групп, [144]). Пусть $G$-сепарабельная аменабельная локально компактная группа, действующая на пространстве с мерой $(X, \mathcal{A}, \nu)$ сохраняющими меру преобразованиями, и пусть $F_{n}$ - умеренная последовательность Фолнера. Тогда для любой функиии $f \in L^{1}(\nu)$ существует такая $G$-инвариантная функиия $\bar{f} \in L^{1}(\nu)$, что

$$
\lim _{n \rightarrow \infty} A\left(F_{n}, f\right)(x)=\bar{f}(x) \quad \text { почти всюду. }
$$

В частности, если действие әруппь $G$ эргодично, то

$$
\lim _{n \rightarrow \infty} A\left(F_{n}, f\right)(x)=\int_{X} f(x) d \nu(x) \text { почти всюду. }
$$

Известно, что некоторое ограничение на последовательность Фолнера $F_{n}$ в этой теореме сушественно, даже при $G=\mathbb{Z}$, но любая последовательность Фолнера имеет умеренную подпоследовательность Фолнера [144]. В частности, любая сепарабельная аменабельная локально компактная группа допускает умеренную последовательность Фолнера.

Перейдем к определению эргодических алгебр функций.

Пусть $G$ - аменабельная локально компактная групп. Измеримая локально интегрируемая функция $\varphi$ на $G$ называется усреднимой к числу $M(\varphi)$, и $M(\varphi)$ назьвается средним значением функции $\varphi$, если последовательность

$$
\mu\left(F_{n}\right)^{-1} \int_{F_{n}} \varphi(g) d \mu(g), \quad n \in \mathbb{N},
$$

сходится к числу $M(\varphi)$ для любой умеренной последовательности Фолнера $F_{n}$ в $G$. Инвариантная относительно сдвигов подалгебра $X$ алгебры $\mathrm{UCB}(G)$ ограниченных равномерно непрерывных функций на $G$ назьвается алгеброй со средним, если $X$ инвариантно относительно сдвига и каждая функция из $X$ усреднима.

Рассмотрим совокупность всех измеримых по мере Хаара функций $\varphi$ на $G$, локально интегрируемых по мере Хаара, для которых $p$-преднорма Безиковича

$$
\|\varphi\|_{p}^{p}=\sup _{\left\{F_{n}\right\}} \limsup _{n \rightarrow \infty}\left(\mu\left(F_{n}\right)\right)^{-1} \int_{F_{n}}|\varphi(g)|^{p} d \mu(g)
$$

конечна, где верхняя грань берется по всем умеренным последовательностям Фолнера $F_{n}$ в $G$. Функция $\varphi$ назьвается $p$-почти периодической по Безиковичу, если она 
является пределом конечных линейных комбинаций матричных элементов непрерьвных неприводимых конечномерных унитарных представлений групшы $G$ по $p$-преднорме Безиковича. Семейство $B^{2}(G)$ функций, 2-почти периодических по Безиковичу, образует евклидово пространство относительно (полу)скалярного произведения, естественно определяемого средним значением. Нетрудно модифицировать известные доказательства полноты пространства $B^{2}\left(\mathbb{R}^{n}\right)[38],[236]$ и доказать полноту полученного евклидова пространства $B^{2}(G)$ (как мы увидим ниже на примере, имеет место нетривиальная зависимость преднорм вида

$$
\limsup _{n \rightarrow \infty}\left(\int_{F_{n}}|\varphi(g)|^{2} d \mu(g)\right)^{1 / 2}
$$

на пространстве $B^{2}(G)$ от выбора умеренной исчерпьвающей монотонной последовательности Фолнера $\left.\left\{F_{n}\right\}\right)$. Заметим, что функция $F$, являюшаяся суммой локально равномерно сходяшегося ряда по матричным элементам непрерывных неприводимых унитарных представлений, является почти периодической функцией Безиковича. Действительно, из построения предельной функции $G$ для подпоследовательности частных сумм ряда, используемой в доказательстве полноты пространства $B^{2}(G)$, следует, что функция $F-G$ стремится к нулю на бесконечности на групе $G$, причем разность $F-G$ можно считать равномерно сколь угодно малой. Следовательно, $F-$ почти периодическая функция по Безиковичу и исходный ряд есть ее ряд Фурье.

В связи с известной характеризащией равномерно непрерьвных функций с единственным средним на сепарабельных аменабельных локально компактных группах [201], равномерной непрерывностью слабо почти периодических функций на групах и единственностью среднего на алгебре слабо почти периодических функций каждая слабо почти периодическая функция на сепарабельной аменабельной локально компактной группе является почти периодической по Безиковичу. Обратное неверно, поскольку функция, почти периодическая по Безиковичу, не обязана иметь единственное среднее (примером может служить функция из примечания к $\S 5$ гл. VII в [236], т.е. функция

$$
f(x)=\sum_{n=1}^{\infty} n^{-1} \sin (x / n), \quad x \in \mathbb{R}
$$

она почти периодична по Безиковичу как сумма локально равномерно сходящегося тригонометрического ряда без свободного члена, но инвариантные средние принимают на этой функции и ненулевые значения, поскольку

$$
T^{-1} \int_{0}^{T} f(x) d x \geqslant c_{0}
$$

для всех $T>1$ и некоторого $\left.c_{0}>0\right)$.

В общем случае замькание $W^{2}$ алгебры со средним $X$ по преднорме Безиковича есть гильбертово пространство, в котором естественно действует группа сдвигов на группе, и это действие непрерывно, поскольку функщии из алгебры равномерно непрерьвны по условию. Алгебра со средним $X$ назьвается эргодической, если всякая инвариантная функция из соответствуюшего пространства $W^{2}$ эквивалентна постоянной, и локально интегрируемая функция $f$ на $G$ назьвается эргодической, если 
она принадлежит пространству $W^{2}$, отвечающему некоторой эргодической алгебре. (В частности, всякая функция, почти периодическая по Безиковичу, эргодична, поскольку сдвиги на групе оставляют на месте только свободньй член ряда Фурье.) В [236] указаны применения эргодических алгебр к теории усреднения дифференциальных операторов.

Пусть $X$ - банахово пространство, $G$ - локально компактная и $\sigma$-компактная абелева группа с левой мерой Хаара $\mu$, а $T$ - слабо почти периодическое представление $G$ в $X$. В [142] показано, что для любой последовательности эргодических вероятностных мер $\left(\mu_{n}\right)$ на $G$ последовательность интегралов

$$
\int w(t) T(t) x d \mu_{n}(t)
$$

сходится сильно для любой 1-почти периодической функции Безиковича $w$ на $G$ и любого $x \in G$ (конечно, для слабо почти периодических функций $w$ предел не зависит от выбора последовательности $\left.\left(\mu_{n}\right)\right)$. Кроме того, из результатов в [142] следует, что для любой умеренной последовательности Фолнера $F_{n}$, любой функции $f \in L^{1}(X, \nu)$ и любой 1-почти периодической функции Безиковича $w$ на $G$ предел

$$
\lim _{n \rightarrow \infty}\left(1 / \mu\left(F_{n}\right)\right) \int_{F_{n}} w(t) f(t x) d \mu(t)
$$

существует почти всюду (если $w$ - слабо почти периодическая функция, то предел не зависит от выбора последовательности $\left.F_{n}\right)$.

Пусть $G$ - локально компактная группа. Последовательность $\left\{\mu_{n}\right\}$ вероятностных мер на $G$ называется сильно (слабо) эргодической, если последовательность $\left\{\pi\left(\mu_{n}\right) \xi\right\}$ сходится по норме (слабо) к элементу пространства $\{\xi \in H: \pi(g) \xi=\xi$ для всех $g \in G\}$ для любого непрерывного неприводимого унитарного представления $\pi$ групшы $G$ в гильбертовом пространстве $H$ и любого $\xi$ в $H$. В [134] получен ряд характеризаций сильных и слабых эргодических последовательностей и показано, что существование сильной или слабой эргодической последовательности равносильно $\sigma$-компактности групшы $G$. Пусть теперь группа $G$ локально компактна и $\sigma$-компактна. Последовательность $\mu_{n}$ вероятностных мер на $G$ назьвается усредняющей, если

$$
\int \pi(g) x d \mu_{n}(g) \rightarrow P_{\pi} x \text { слабо при } n \rightarrow \infty
$$

для любого $x \in X$ и любого слабо почти периодического представления $\pi$ групшы $G$ в банаховом пространстве $X$, где $P_{\pi}$ - эргодический проектор, отображающий пространство $X$ на подпространство $\pi$-инвариантных векторов. В [143] доказано следуюшее утверждение о связи между сильньм и слабым усреднением: если $\left\{\mu_{n}\right\}$ - усредняюшая последовательность мер и $f$ - такая измеримая функция на $G$, что

$$
\limsup _{n} \int|f|^{r} d \mu_{n}<\infty \text { для некоторого } r>1,
$$

то последовательность интегралов

$$
\int f(g) \pi(g) x d \mu_{n}(g)
$$


сходится сильно для любого слабо почти периодического представления $\pi$ и любого вектора $x$ в пространстве представления $\pi$ тогда и только тогда, когда последовательность интегралов

$$
\int f(g) l(g) d \mu_{n}(g)
$$

сходится для любой почти периодической функции $l$ на $G$. Более специальные результаты получены для случая, когда $f$ - почти периодическая функция Безиковича ${ }^{11}$.

В теории дифференциальных уравнений полезен также класс функций, имеющих единственное модульное среднее над почти периодическими функциями (это условие также является условием типа эргодичности [22]). А именно, если $G$-топологическая групп, $E$ - локально вьпуклое пространство и $M$ - такое векторное подпространство

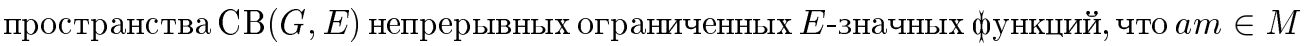
для любых $m \in M$ и любых скалярных почти периодических функций $a$ на $G$, то говорят, что $M$ допускает единственное модульное среднее значение, если все скалярные функции вида $a F(m)$, где $F \in E^{*}, a \in A P(G), m \in M$, имеют единственное инвариантное среднее значение в обычном смысле. (Это условие можно также выразить в терминах векторнозначных средних (ср. [230]).) Иногда удается заменить этим метрическим свойством условие слабой компактности семейства сдвигов с сохранением характера утверждений [25]. Попытки проделать работу этого рода для рекуррентных и так называемых эргодических функций с помошю эргодических теорем предприняты в [65], где аналоги разложения Эберлейна получены за пределами класса слабо почти периодических функций. Свойства почти периодических точек динамической системы рассматриваются в [178]. Почти периодические функции в связи с динамическими системами на более общих топологических пространствах рассматривались в [170], что позволило унифицировать доказательства ряда известных теорем.

Функция на локально компактной группе, на которой все левоинвариантные средние (если $G$ - некомпактная локально компактная группа, то на пространстве $L^{\infty}(G)$ заведомо имеется несчетно много левоинвариантных средних) принимают общее значение, назьвается почти сходящейся слева (и это свойство не совпадает с введенной вьше усреднимостью). В [186] приведена характеризация почти сходяшихся функций на группах, аменабельных в дискретной топологии (например, разрешимых). Справедливость этой характеризации может служить критерием того, что группа аменабельна в дискретной топологии [201]. Если $G$ - бесконечная компактная група, то множество инвариантных средних на пространстве $C(G)$ одноэлементно (в силу единственности нормированной меры Хаара), а множество инвариантных средних на пространстве $L^{\infty}(G)$ может содержать и несчетное семейство элементов (подробности можно найти в [177]), а может быть одноэлементным (см. [58] и п. 8.2 ниже), и в последнем случае все функции из $L^{\infty}(G)$ являются почти сходяшимися слева. В любом случае, кроме непрерьвных функций, почти сходяшимися функциями на групе $G$ являются и все функции, интегрируемые по Риману по мере Хаара. В [209] для метризуемых компактных групп указаны достаточные условия того, что почти сходяшиеся функции на данной компактной групе не исчерпьваются функциями, интегрируемыми по Риману по мере Хаapa.

\footnotetext{
${ }^{11}$ Описание эргодических (усреднимых) векторов в банаховом пространстве представления полугруппы приведено в статье автора "Равномерное распределение, инвариантные средние и интеграл Римана" (Матем. заметки. 1994. Т. 56. № 3. С. 144-154).
} 
Семейство ограниченных непрерьвных функций с единственным средним на некомпактной локально компактной группе не образует алгебру, и для этого пространства нет аналога разложения Эберлейна, но можно ожидать, что подалгебры в этом пространстве допускают такое разложение. Предварительные результаты в этом направлении получены в [199], [201].

Отметим, что почти периодические функции Бора на аменабельной локально компактной групе почти сходятся слева [164].

Родственньй класс образуют дистальные функции, но мы здесь не касаемся обобщений понятия дистальности на случай функций на группе со значениями в локально вьпуклых пространствах (напомним, что действие топологической группы $G$ (с единицей $e$ ) на топологическом пространстве $X$ - это такое непрерьвное отображение $\alpha: G \times X \rightarrow X$, что $\alpha(e, x)=x$ для всех $x \in X$ и $\alpha\left(g_{1} g_{2}, x\right)=\alpha\left(g_{1}, \alpha\left(g_{2}, x\right)\right)$ для всех $g_{1}, g_{2} \in G$ и всех $x \in X$ (тройка $\{X, G, \alpha\}$ назьвается $G$-пространством), действие

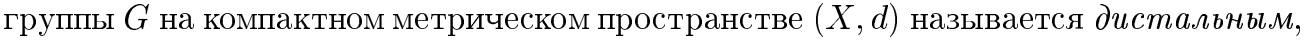
если для любых различных $x, y \in X$ сушествует такое $\varepsilon>0$, что $d(g x, g y)>\varepsilon$ для всех $g \in G$, а функция назьвается дистальной, если замыкание ее орбиты компактно и действие группы на этом компакте дистально). Класс дистальных функций и его связи с почти периодическими функциями обсуждаются, в частности, в [37], [121], [108], [136], [157]-[166]. Отметим еще два факта, связывающие рассматриваемые явления с топологической динамикой. Если $G$ - польская (полная сепарабельная метризуемая) группа, то любое польское $G$-пространство можно (топологически) вложить в компактное польское $G$-пространство тогда и только тогда, когда групп $G$ локально компактна [194]. Кроме того, напомним, что действие $\alpha$ топологической групшы $G$ на компактном пространстве $X$ называется слабо почти периодическим, если $\alpha$-орбита любой непрерьвной функции на $X$ слабо относительно компактна в $C(X)$. В $[155]$ показано, что топологическая группа $G$ предкомпактна (т.е. топологически изоморфна подгруппе компактной групшы) тогда и только тогда, когда любое непрерьвное действие групшы $G$ на компактном пространстве слабо почти периодично.

Класс почти периодических функций на полутопологических полугруппах существенно отличается от соответствуюшего класса на топологических группах и, в частности, это различие сушественно на уровне существования средних. А именно, если $S$ - полутопологическая полугруппа и $A P(S)$ - пространство почти периодических функций на $S$, то левоинвариантное среднее на $A P(S)$ существует тогда и только тогда, когда любое раздельно непрерывное, равностепенно непрерьвное и аффинное действие полугрупшы $S$ на компактном вьпуклом подмножестве $K$ отделимого локально вьпуклого пространства имеет общую неподвижную точку в $K$ (см. [132]).

Разложение Эберлейна, являющееся центральным вопросом теории слабо почти периодических функций и одной из основных тем настоящего обзора, имеет многообразные приложения всюду, где возникает понятие почти периодичности. В частности, это разложение было успешно применено при изучении структуры почти периодических мультипликаторов (как элементов пространства $\left.L^{\infty}(G)\right)$ из пространства $L^{1}(G)$ в пространство $L^{\infty}(G)$ на локально компактной абелевой группе $G$ в сильной или слабой операторной топологии в [64] как мультипликаторов, отображающих единичньй шар пространства $L^{1}(G)$ в относительно компактное подмножество в $L^{\infty}(G)$ и при изучении мультипликаторов Ганкеля-Шура в пространстве Харди [3]. 


\section{§ 8. Другие направления исследований,} связанные с почти периодическими функциями

Круг вопросов, связанных с теорией почти периодических функций и представлений, далеко не сводится к перечисленным вьше классическим задачам. Мы кратко опишем некоторые современные направления исследований в этой области.

\section{1. Компактификация Бора, [MAP] группы и [FIR] группы.}

Топологическаягруппа назьвается максимальной почти периодической ([MAP]), если она имеет достаточно много топологически неприводимых представлений в конечномерных гильбертовых пространствах. Строение групп этого класса известно для связных локально компактных топологических групп еше с конца тридщатых годов, но даже в классе общих локально компактных групп полного описания этого класса нет. Замькание $K$ образа группы в прямой сумме всех топологически неприводимых непрерьвных представлений данной топологической групшы $G$ в конечномерных гильбертовых пространствах есть компактная топологическая группа, так называемая боровская компактификация групшы $G$. Отметим, что числовые почти периодические функции на $G$ являются в точности ограничениями на $G$ непрерьвных функций на $K$ (это было по существу отмечено уже в [222]), и было бы интересно выяснить условия полноты в классе локально выпуклых пространств, для которых почти периодические функции на $G$ со значениями в этих пространствах допускают автоматическое продолжение до непрерьвных функций на группе $K$. Вопросы продолжения числовых почти периодических функций с (в том числе плотных) подполугруп полутопологических полугрупп рассматривались, в частности, в [32], [47].

Свойствам боровских компактификаций и, в частности, связям между свойствами групш $G$ и $K$ посвяшено много работ; см. обзорную статью [60]. Отметим, что из свойства сохранения компактности для локально компактных абелевых групп (класс множеств, компактных в исходной топологии, совпадает с классом множеств, компактных в топологии, определяемой почти периодическими функциями на групе [89]) следует, что счетная на бесконечности локально компактная абелева групша является множеством типа $F_{\sigma}$ в своей боровской компактификации, а произвольная локально компактная абелева група является борелевским подмножеством своей боровской компактификации $K[60]$, и это подмножество имеет нулевую меру Хаара на $K$, если исходная группа $G$ некомпактна. С помошю результатов статьи [182] о сохранении компактности при переходе к боровской компактификации для так назьваемых [FIR] групп (локально компактных груп, все топологически неприводимые унитарные представления которых конечномерны) оба утверждения допускают распространение на этот случай. Таким образом, счетная на бесконечности локально компактная [FIR] группа является множеством типа $F_{\sigma}$ в своей боровской компактификации, а произвольная локально компактная [FIR] группа является борелевским подмножсеством своей боровской компактификации, и это подмножество имеет нулевую меру Хаара на $K$, если исходная группа $G$ некомпактна. Свойство сохранения компактности при переходе к боровской компактификации вьполняется и для более обших топологических абелевых групп [111]. 
8.2. Геометрия групповых алгебр. Свойства Радона-Никодима, Данфорда-Петтиса, Асплунда, Шура.

Мы уже встречались со свойством Шура для банаховых пространств. Напомним теперь определения других полезных геометрических свойств.

ОПРЕДЕЛЕНИЕ 11. Пусть $T$ - топологическое пространство, $d$-некоторая метрика на $T$. Пространство $T$ назьвается фрагментируемым $с$ помощью метрики $d$, если любое подмножество пространства $T$ содержит непустые относительно открытые подмножества сколь угодно малого диаметра относительно метрики $d$.

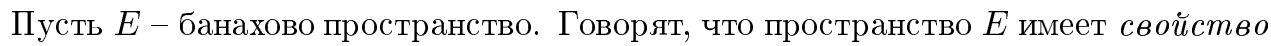
Радона-Никодима, если для любого ограниченного множества $A \subset E$ существуют его подмножества вида $\{\xi \in A \mid f(x)>\sup f(A)-\varepsilon\}$, где $f \in E^{*}$ и $\varepsilon>0$, имеющие сколь угодно мальй диаметр. Говорят, что пространство $E$ имеет свойство Крейна-Мильмана, если любое ограниченное замкнутое вьпуклое подмножество в $E$ есть замкнутая вьпуклая оболочка множества своих крайних точек. Говорят, что пространство $E$ имеет свойство Данфорда-Петтиса, если $f_{n}\left(x_{n}\right) \rightarrow 0$ для любой слабо стремяшейся к нулю последовательности $\left\{\xi_{n}\right\}$ в $E$ и любой слабо стремящейся

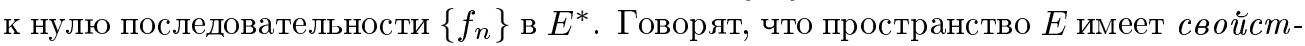
во Асплунда, если любое сепарабельное подпространство в $E$ имеет сепарабельное сопряженное пространство.

Эти свойства тесно связаны между собой, и каждое из них имеет много эквивалентных вариантов, подробно описанных в [70], [71], [226]. Известно, например, что следуюшие условия равносильны для любого банахова пространства $E$ :

(a) $E$ имеет свойство Асплунда,

(б) единичный шар в $E^{*}$, рассматриваемьй в слабой* топологии, фрагментируем относительно метрики, определяемой нормой,

(в) $E^{*}$ имеет свойство Крейна-Мильмана,

(г) $E^{*}$ имеет свойство Радона-Никодима.

Как мы только что видели, [MAР] групшы - это группы, на которых семейство неприводимых непрерьвных конечномерных представлений разделяет точки групш, или, что то же, семейство непрерьвных почти периодических функций разделяет точки групшы. Любое непрерьвное унитарное представление топологической групшы можно рассматривать как отображение в замкнутьй единичный шар пространства непрерьвных линейных операторов в пространстве представления, а этот шар можно рассматривать как компактную полутопологическую полугруппу в слабой операторной топологии. Поэтому все матричные элементы всех непрерьвных унитарных представлений являются слабо почти периодическими функциями на групе. Их линейные комбинации образуют (относительно поточечного умножения) так называемую алгебру Фурье-Cтилтьеса $B(G)$ данной топологической групшы $G$. Таким образом, $B(G) \subset W A P(G)$. Если группа $G$ локально компактна, то семейство матричных элементов ее регулярного представления образует подалгебру $A(G)$ алгебры $B(G)$, так назьваемую алгебру Фурье локально компактной группы $G$. Если $G-$ коммутативная локально компактная группа, то алгебра Фурье $A(G)$ изоморфна групповой алгебре $L^{1}(\widehat{G})$ группы характеров группы $G$. Основным руководством по 
алгебрам Фурье и Фурье-Стилтьеса локально компактных груп является статья Эймара [82]. Некоторые компактификации, связанные с операторными пространствами, определяемыми непрерьвными унитарными представлениями, изучены в [225].

Принадлежность классу [FIR] (и даже более общему классу [MAP] топологических груп, все непрерьвные унитарные представления которых принадлежат типу I и являются прямыми суммами представлений конечного типа) может быть охарактеризована в геометрических терминах [204; замечание 2].

Теорема М. Пусть G-топологическая группа, семейство унитарных представлений которой разделяет точки группь. Тогда следующие условия равносильны:

(1) группа $G$ допускает вложение в компактную группу, и любое непрерывное унитарное представление группы $G$ определяется непрерывным унитарным представлением этой компактной группь;

(2) алгебра Фурье-Стилтьеса $B(G)$ группь $G$ одновременно имеет свойство Данфорда-Петтиса и свойство Радона-Никодима.

Таким образом, геометрические свойства групповой алгебры оказьваются связаны с теорией матричных элементов непрерьвных представлений группы или, что то же, с теорией почти периодических функщий на этой групе. Приведем некоторые известные геометрические утверждения о груповых алгебрах.

В [96] рассматриваются свойства банаховых факторпространств алгебры Фурье $A(G)$. В частности, показано, что если $J$ - замкнутьй идеал в $A(G)$ с множеством нулей $F \subseteq G$ и если сушествует такая замкнутая подгруппа $H$ в $G$, аменабельная как дискретная групп, что $F \cap H$ содержит совершенное множество, то факторалгебра $A(G) / J$ содержит копию пространства $l^{1}$. Отсюда следует, что в пространстве, сопряженном к $A(G) / J$, не вьполняется ни свойство Радона-Никодима, ни свойство Шура. Напротив, известно, что если група $G$ абелева и множество $F$ компактно и разрежено, то $A(G) / J$ не содержит копии пространства $l^{1}$, причем сопряженное пространство $(A(G) / J)^{*}$ имеет и свойство Радона-Никодима, и свойство Шура.

Пусть $G$ - локально компактная группа и $V N(G)$ - алгебра фон Неймана, порожденная левым регулярным представлением групшы $G$. Тогда алгебра Фурье $A(G)$ групшы $G$ естественно отождествляется с преддвойственным пространством алгебры фон Неймана $V N(G)$, и $V N(G)$ является модулем над $A(G)$ относительно операции $\langle u \cdot T, v\rangle=\langle T, u v\rangle, T \in V N(G), u, v \in A(G)$.

В [30] символ $U C B(\widehat{G})$ обозначает подпространство в $V N(G)$, порожденное элементами $\{u \cdot T: u \in A(G), T \in V N(G)\}$. Если $T \in V N(G)$ и множество $\{u \cdot T:$ $\left.\|u\|_{A(G)} \leqslant 1\right\}$ относительно (слабо) компактно в $V N(G)$, то элемент $T$ назьвается (слабо) почти периодическим. Пусть $A P(\widehat{G})$ (соответственно $W A P(\widehat{G})$ ) обозначает пространство всех почти периодических (соответственно слабо почти периодических) элементов в $V N(G)$. При $x \in G$ обозначим через $\lambda_{x}$ оператор левого сдвига на $x$ в $L^{2}(G)$. Пусть $C_{\delta}^{*}(G)$ обозначает $C^{*}$-алгебру, порожденную семейством $\left\{\lambda_{x}: x \in G\right\}$. Тогда $C_{\delta}^{*}(G) \subseteq A P(\widehat{G}) \subseteq W A P(\widehat{G})$ и $C_{\delta}^{*}(G) \subseteq U C B(\widehat{G})$. В [30] доказано, что следующие условия равносильны: (группа $G$ конечна) $\Longleftrightarrow\left(C^{*}\right.$-алгебра $U C B(\widehat{G})$ разрежена $) \Longleftrightarrow(W A P(\widehat{G})$ имеет свойство Асплунда $) \Longleftrightarrow(A P(\widehat{G})$ имеет свойство Асплунда $) \Longleftrightarrow\left(C^{*}\right.$-алгебра $C_{\delta}^{*}(G)$ разрежена), и что следуюшие условия также равносильны: $(V N(G)$ имеет свойство Данфорда-Петтиса) $\Longleftrightarrow(U C B(\widehat{G})$ имеет свойство 
Данфорда-Петтиса $) \Longleftrightarrow\left(C_{\delta}^{*}(G)\right.$ имеет свойство Данфорда-Петтиса $) \Longleftrightarrow(G$ имеет абелеву подгрупу конечного индекса). Эквивалентность $(V N(G)$ имеет свойство Данфорда-Петтиса $) \Longleftrightarrow(G$ имеет абелеву подгрупшу конечного индекса $)$ имеет место для любой унитарно представимой топологической группы [204].

В [56] локально компактная некомпактная групша $G$ назьвается группой Эберлейна, если любая непрерывная слабо почти периодическая функция на $G$ может быть равномерно приближена функциями из $B(G)$. Показано, что если $G$ нильпотентна или если в $G$ имеется инвариантная компактная окрестность единичного элемента, то $G$ не является групой Эберлейна. Абелевы групш тоже не являются группами Эберлейна. Примерами груп Эберлейна являются конечные прямые произведения евклидовых групп движений и некомпактные полупростые групш Ли с конечньм центром. В [54] рассматривается связанная с этим задача о структуре равномерного замыкания алгебры Фурье-Стилтьеса $B(G)$ локально компактной групшы $G$. Пусть $H$ - замкнутьй нормальный делитель в $G$ и $f$ - непрерывная функция на $G$, постоянная на классах смежности $x H, x \in G$. Показано, что если $f$ может быть равномерно приближена функциями из $B(G)$ (заметим, что функция $f$ автоматически слабо почти периодична), то она может быть равномерно приближена функциями из $B(G)$, постоянньми на классах смежности вида $x H, x \in G$. Доказательство этого факта использует единственное инвариантное относительно сдвигов среднее на пространстве непрерьвных слабо почти периодических функций на $H$. Если $h \in B(G)$ и $\|f-g\|_{\infty}<\varepsilon$, то функция $h^{\prime}$, получаемая усреднением функции $h$ на каждом классе смежности, непрерьвна (вследствие равномерной непрерьвности функций из $B(G)$ ) и, более того, принадлежит алгебре $B(G)$, причем $\left\|f-h^{\prime}\right\|_{\infty}<\varepsilon$.

В [58] через $A P(\widehat{G})$ обозначено множество всех таких $T \in V N(G)$, что оператор $u \mapsto u \cdot T$ из $A(G)$ в $V N(G)$ компактен, а через $C_{\delta}^{*}(G)$ обозначена $C^{*}$-алгебра, порожденная семейством $\{\lambda(x) \mid x \in G\}$. Известно, что $C_{\delta}^{*}(G) \subset A P(\widehat{G})$, причем $C_{\delta}^{*}(G)=A P(\widehat{G})$ (его иногда назьвают свойством дуальной аппроксимачии Бора, поскольку в абелевом случае $C^{*}$-алгебра $C_{\delta}^{*}(G)$ совпадает с равномерным замьканием тригонометрических многочленов на группе характеров $\widehat{G}$ группы $G)$, если $G-$ локально компактная абелева группа или дискретная аменабельная группа. Показано, что любое расширение локально компактной абелевой группы с помошью аменабельной дискретной группы тоже имеет свойство дуальной аппроксимации Бора, и сушествует такая бесконечная компактная групша $G$, что $C_{\delta}^{*}(G) \subsetneq A P(\widehat{G})$. Если $G$ - компактная группа, то одномерньй оператор $E$ в $L^{2}(G)$, определенньй формулой $E(f)=\left(\int f(t) d t\right) 1, f \in L^{2}(G)$, удовлетворяет условию $E \in C_{\delta}^{*}(G)$ тогда и только тогда, ког да $L^{\infty}(G)$ имеет единственное левоинвариантное среднее, или тогда и только тогда, когда $G$ полупроста [58], [59].

Родственный вопрос о структуре ограничений функциональных пространств на локально компактной групе на некоторую замкнутую полугруппу рассматривался в [61]. Пусть $G$ - локально компактная групша и $E(G)$ - либо пространство ограниченных лево- и право-равномерно непрерывных функций на $G$, либо пространство слабо почти периодических функций на $G$, либо алгебра Фурье-Стилтьеса группы $G$. Если $H$ - замкнутая подгруппа в $G$, то, вообше говоря, семейство ограничений $\left.E(G)\right|_{H}$ является правильной частью $E(H)$. Получено изящное необходимое и достаточное условие того, что функция из $E(H)$ принадлежит $\left.E(G)\right|_{H}$, если $H$ - нормальный делитель. Более точно, если $N$ - замкнутый нормальньй делитель в $G, x \in E(N)$ 
и $g \in G$, то можно определить элемент $x^{g} \in E(N)$ формулой $x^{g}(n)=x\left(g^{-1} n g\right)$. Показано, что функция $x$ из $E(N)$ принадлежит $\left.E(G)\right|_{N}$ тогда и только тогда, когда отображение $g \rightarrow x^{g}$ групшы $G$ в $E(N)$ непрерывно в точке $e \in G$. Отсюда можно вьвести много утверждений о продолжениях функций из $E(N)$. Кроме того, показано, что если $H$ - замкнутая подгруппа [SIN]-группы $G$ (т.е. локально компактной групшы, имеющей фундаментальную систему окрестностей единичного элемента, инвариантных относительно внутренних автоморфизмов), то $\left.E(G)\right|_{H}=E(H)$. В частности, если $G$ - связная группа Ли, то (1) $\left.E(G)\right|_{N}=E(N)$ для любого замкнутого нормального делителя $N$ в $G$ тогда и только тогда, когда радикал групшы $G$ централен, (2) $\left.E(G)\right|_{H}=E(H)$ для любой замкнутой подгрупшы $H$ в $G$ тогда и только тогда, когда $G$ - прямое произведение векторной группы и компактной группы.

Подмножество $E$ локально компактной абелевой группы $G$ называется $I$-множеством, если любая ограниченная комплексная функция на $E$ может быть продолжена до почти периодической функции на $G$ (или, что равносильно, до непрерьвной функшии на компактификации Бора группы $G$ ). Следующие утверждения доказаны в [94]. Если $G$ - дискретная абелева группа и $K$ - компактификация Бора групшы $G$, то сушествует точка $y_{0} \in K \backslash G$, не принадлежашая $K$-замыканию никакого $I$-множества $E$ в $G$; кроме того, любая дискретная абелева группа $G$ содержит $I$-множество, порождающее групп $G$. Интересно, справедливы ли эти утверждения для неабелевых дискретных групп.

Структура замыканий алгебр Фурье-Стилтьеса связана также с асимптотическим поведением матричных элементов. Локально компактная група называется минимальной, если любой непрерьвньй изоморфизм этой группы на любую топологическую групу открыт, и вполне минимальной, если любой сюръективный непрерывньй гомоморфизм открыт. Основным руководством по теории минимальных топологических групп является книга [72]. Теория минимальных групп связана с асимптотическим поведением матричных элементов представлений этих групп. В [152] показано, что связные вполне минимальные группы - это групшы с компактным нормальньм делителем, факторгруппа по которому является полупрямым произведением односвязной нильпотентной групшы и связной редуктивной группы, действуюшей на нильпотентной части без нетривиальных неподвижных точек. Кроме того, получены некоторые результаты о стремлении к нулю на бесконечности матричных элементов представлений минимальной группы и показано, что для связных групп условие полной минимальности равносильно свойству Эберлейна, обсужденному вьше (равномерное замькание алгебры Фурье-Стилтьеса матричных элементов унитарных непрерывных представлений есть вся $C^{*}$-алгебра слабо почти периодических функций).

Слабо почти периодические компактификации (см. теорему D) минимальных топологических групा изучены в [151]. Установлено соответствие между идемпотентами в $w(G)$ и замкнутыми нормальными делителями в $G$. Это соответствие основано на наблюдении, что пространство $w(G)$ можно разложить на $G$-орбиты идемпотентов в $w(G)$, если $G$ вполне минимальна. Отсюда следует, что каждое непрерывное унитарное представление такой группы разлагается в ортогональную сумму двух представлений - конечномерного и (по модулю ядра) сильно перемешивающего.

8.3. Свойства слабо почти периодических компактификаций групп и полугрупп.

Полугруповой компактификацией полутопологической полугрупшы $T$ назьвается 
пара $(\varepsilon, S)$, где $S$ - компактная полутопологическая полугрупп, а $\varepsilon$ - непрерьвный гомоморфизм полугрупшы $T$ в $S$ с плотньм образом. Одной из полугрупповых компактификаций является пространство $T^{\mathscr{W} \mathscr{A}}$ максимальных идеалов алгебры слабо почти периодических функций на $T$ (с естественным отображением $\varepsilon$ ), т.е. введенная в теореме D слабо почти периодическая компактификация полутопологической полугрупшы $T$, и это - наибольшая из ее полугрупповых компактификаций (см. теорему D и $[36],[37],[88],[135])$.

Пусть $G$ - локально компактная группа и $N$ - замкнутьй нормальньй делитель в $G$ с компактной факторгруппй $G / N$. Полугрупповая компактификация $X$ группы $N$ назьвается согласованной с группой $G$, если любой автоморфизм нормального делителя $N$, определяемьй сопряжением с элементом групшы $G$, продолжается до непрерьвного отображения пространства $X$ в себя. В [135] показано, что слабо почти периодическая компактификация нормального делителя сдвигов в групе $G$ движений плоскости не согласована с $G$. С другой стороны, любая полугруповая компактификация замкнутой подгрупшы любой абелевой групшы $G$ согласована с $G$ (этот факт позволяет получить явное описание структуры слабо почти периодической компактификации числовой прямой [135]).

Пусть $G$ - топологическая группа, $G^{\mathscr{L} \mathscr{U} \mathscr{C}}$ - ее $L U C$-компактификация (см. п. 9.5), a $G^{\mathscr{W A P}}$ - WAP-компактификация. Согласно знаменитой теореме Вича (см. [37] и $[216])$, если $G$ - локально компактная группа с единичным элементом $e$, то $g x \neq x$ для любого $x \in G^{\mathscr{L} \mathscr{U} \mathscr{C}}$ и любого $g \in G, g \neq e$ (в частности, любая локально компактная групша свободно действует на некотором компакте). Следующий результат является $W A P$-аналогом теоремы Вича. В [84] показано, что если $G$ - [SIN]-группа, и если рассматривать полугрупшу $G^{\mathscr{W} \mathscr{A} \mathscr{P}}$ как фактор правой полугруппы $G^{\mathscr{L} \mathscr{U} \mathscr{C}}$ (определяемой ниже в этом разделе), то существует открытое плотное подмножество в $G^{\mathscr{L} \mathscr{U} \mathscr{C}} \backslash G$, состоящее из точек единственности для отображения факторизации (но это свойство может не выполняться для IN-групп).

B [34] изучается связь между полутопологической полугруппой и ее слабо почти периодической компактификацией. Вообще говоря, полученная при этой компактификации компактная полугруппа не является топологической компактификацией исходной полугруппы, так как каноническое вложение не всегда оказьвается гомеоморфизмом на образ вложения. Получены достаточные условия того, что почти периодическая компактификация данной локально компактной полутопологической полугруппы является топологической компактификацией. В частности, для многих дискретных полугруп слабо почти периодическая компактификация является топологической. Показано также, что слабая почти периодическая компактификация любой замкнутой подполугруппы локально компактной группы является топологической компактификацией. Кроме того, получено описание слабо почти периодических функций на замкнутой подполугруппе топологической группы, описаны классы слабо почти периодических функций на группе целых чисел $\mathbb{Z}$ и на натуральном ряде $\mathbb{N}$ и показано на примере, что слабо почти периодическая компактификация полугрупы $\mathbb{N}$ не является замьканием множества $\mathbb{N}$ в слабо почти периодической компактификации группы $\mathbb{Z}$.

По мере накопления подобных патологических фактов становилось ясно, что слабо почти периодическая компактификация сохраняет большой объем информации об исходной некомпактной группе или полугруппе и отражает специфику объекта в форме патологических свойств. Тем не менее результат работы [48], вьнесенньй в ее заго- 
ловок, вызвал сильный интерес. Пусть $w \mathbb{Z}$ - слабо почти периодическая компактификация аддитивной групы целых чисел $\mathbb{Z} ; w \mathbb{Z}$ - компактная хаусдорфова полутопологическая полугрупп, содержашая плотную копию группы $\mathbb{Z}$ и являющаяся наибольшей в том смысле, что любая полутопологическая полугрупповая компактификация группы $\mathbb{Z}$ есть непрерьвная гомоморфная копия $w \mathbb{Z}$. Трудности изучения полугруппы $w \mathbb{Z}$ были связаны прежде всего с отсутствием набора нетривиальных примеров ограниченных слабо почти периодических функций на $\mathbb{Z}$; напомним, что ограниченная функщия $f$ на $\mathbb{Z}$ слабо почти периодична тогда и только тогда, когда для любых последовательностей $\left(x_{n}\right)$ и $\left(y_{m}\right)$ в $\mathbb{Z}$ выполняется предельное соотношение

$$
\lim _{n \rightarrow \infty} \lim _{m \rightarrow \infty} f\left(x_{n}+y_{m}\right)=\lim _{m \rightarrow \infty} \lim _{n \rightarrow \infty} f\left(x_{n}+y_{m}\right),
$$

если эти повторные пределы существуют. Положение изменилось с появлением работы [190], в которой были построены новые ограниченные слабо почти периодические функции, поскольку до нее единственными примерами слабо почти периодических функций были функции, стремящиеся к нулю на бесконечности, почти периодические функции и функции с носителями в тонких множествах (например, множествах Сидона) $[55]$.

Компактная полутопологическая полугруппа $w \mathbb{Z}$ имеет $2^{\mathfrak{c}}$ различных идемпотентов. С помощью функций Рупперта [190] удалось доказать, что семейство этих идемпотентов не является замкнутым. Следуюшие факты сушественны. Во-первых, слабо почти периодическая функция на $\mathbb{Z}$ может быть не ограниченной, но ограниченная функция на $\mathbb{Z}$ со значениями в $[0, \infty]$ имеет непрерьвное продолжение на $w \mathbb{Z}$ тогда и только тогда, когда эта функция слабо почти периодична. Во-вторых, любое целое число $x \in \mathbb{Z}$ имеет единственное разложение в виде

$$
x=\sum_{i \in \omega} x(i)(-2)^{i},
$$

где $x(i)=1$ на не более чем конечном множестве индексов $i$ и $x(i)=0$ в остальных точках. Показано, что если $c$ - невозрастающая последовательность неотрищательных вешественных чисел, стремящаяся к нулю, то функция $g_{c}$ на $\mathbb{Z}$ со значениями в $[0, \infty]$, определенная формулой

$$
g_{c}(x)=\sum_{i \in \omega} c(i) x(i)
$$

слабо почти периодична. Рассмотрим компактную подполугруппу

$$
H=\bigcap_{n=1}^{\infty} \overline{\{x: \operatorname{supp} x \geqslant n\}}
$$

полугрупшы $w \mathbb{Z}$, где $\operatorname{supp} x=\{i: x(i) \neq 0\}$. Пусть $\Gamma$ - счетное множество дизъюнктных бесконечных строго возрастаюших последовательностей в $\omega$. Для любого $\gamma \in$ Г определен соответствуюший идемпотент $e^{\gamma}$ в $H$ и некоторая специальная последовательность $c^{\gamma}$, для которой слабо почти периодическая функщия $g_{c} \gamma$ (определенная формулой (11)) удовлетворяет условию

$$
g_{c^{\gamma}}\left(e^{\eta}\right)= \begin{cases}\infty, & \text { если } \gamma=\eta \\ 0, & \text { если } \gamma \neq \eta\end{cases}
$$


Тогда функция

$$
G(x)=\sum_{\gamma \in \Gamma}\left(g^{\gamma} \wedge 1\right)
$$

определенная на $\mathbb{Z}$, где $g^{\gamma} \wedge 1-$ функция, определенная на $w \mathbb{Z}$ формулой

$$
\left(g^{\gamma} \wedge 1\right)(x)=\min \left\{g^{\gamma}(x), 1\right\} \text {, }
$$

оказьвается слабо почти периодической функцией.

Пусть $\xi$ - некоторая точка прикосновения множества $\left(e^{\gamma}\right)$. Можно показать, что $G(\xi)=1$, но $G(\xi+\xi)=2$, потому что $g^{\gamma}$ - гомоморфизм на $H$. Таким образом, $\xi$ - не идемпотент, и тем самым множество идемпотентов в $w \mathbb{Z}$ не замкнуто. Аналогичный результат справедлив для $w \mathbb{N}$.

Правой полугрупповой компактификацией (бесконечной) локально компактной групшы $G$ назьвается пара $(\varepsilon, S)$, где $S$ - такая полугруппа с компактной топологией, что отображение $s \mapsto s t, s \in S$, непрерьвно для любого $t$ в $S$, а $\varepsilon$ - такой непрерьвный гомоморфизм групшы $G$ в $S$ с плотным образом, что отображение $s \mapsto \varepsilon(g) s, s \in S$, непрерьвно для любого $g \in G$ [181]. Одной из правых полугрупповых компактификаций является пространство $G^{\mathscr{L}^{\mathscr{C}} \mathscr{C}}$ максимальных идеалов алгебры равномерно непрерьвных слева ограниченных функций на $G$ с естественным отображением $\varepsilon$ (и это - наибольшая из правых полугрупповых компактификаций). Как показано в [137], каждьй минимальньй левьй идеал в $S$ есть алгебраическое прямое произведение $L=E \times H$, где $H$ - группа и $E$ - множество идемпотентов в $L$ (один из идемпотентов, $e_{0}$, есть единица групшы $H$ ). Оказьвается, $L$ никогда не является группой, так что $E$ содержит более одного элемента, а если $G$ почти связна или боровская компактификация групшы $G$ бесконечна, то множество $E$ содержит подмножество, равномощное множеству подмножеств прямой и группа $H$ незамкнута.

\section{$\S$ 9. Близкие задачи и приложения}

\section{1. Геометрия банаховых и локально выпуклых пространств.}

Определение почти периодических и слабо почти периодических представлений и функций включает условия относительной компактности и слабой относительной компактности орбит и потому связано с условиями относительной компактности и слабой относительной компактности в пространствах векторных функций. В качестве примера приведем некоторые общие результаты об условиях слабой компактности в пространстве $L^{\infty}(\mu, X)$, где $X$ - банахово пространство. В [4] показано, что если $X-$ банахово пространство со свойством Шура и $\mu$ - конечная мера, то слабо сходяшиеся последовательности в $L^{\infty}(\mu, X)$ сходятся по норме почти всюду, любое рефлексивное подпространство в $L^{\infty}(\mu, X)$, на котором топологии пространств $L^{p}(\mu, X)$ и $L^{\infty}(\mu, X)$ согласованыпри некотором $p, 1 \leqslant p<\infty$, конечномерно, и если $W$-слабо компактное подмножество в $L^{\infty}(\mu, X)$, то для любого $\varepsilon>0$ сушествует такое множество $E$, мера дополнения которого $<\varepsilon$, что множество $\left\{\left.f\right|_{E} \mid f \in W\right\}$ относительно компактно в $L^{\infty}(\mu, X)$. Важно отметить, что условие Шура необходимо для справедливости этих утверждений.

В [133] изучались вопросы дополняемости некоторых подпространств пространств $L^{\infty}(G)$ и $L U C(G)$ для локально компактной групшы $G$. В частности, показано, что 
(1) замкнутое подпространство пространства $W A P(G)(\mathrm{CB}(G))$, содержащее $C_{0}(G)$, не дополняемо в $L^{\infty}(G)$, если группа $G$ бесконечна (недискретна), $(2)$ если $G$ некомпактна, то $W A P(G)$ не дополняема в $L U C(G),(3)$ пространство $A P(G)$ дополняемо в $L U C(G)$ тогда и только тогда, когда $G / N$ компактна, где $N$ - ядро естественного гомоморфизма группы $G$ в ее компактификацию Бора. Доказательство использует существование сжимающего линейного проектора пространства $\mathrm{CB}(G)$ на пространство $\mathrm{CB}(G / H)$, где $H$ - замкнутая подгруппа в $G$. Факторпространства, связанные с естественными включениями, часто несепарабельны (например, факторпространство пространства ограниченных равномерно непрерьвных функций на некомпактной локально компактной группе по подпространству слабо почти периодических функций при некоторых условия содержит линейную изометрическую копию пространства $l^{\infty}$ ); с другой стороны, есть некомпактные локально компактные групшы, для которых запас диссипативных слабо почти периодических функций состоит только из непрерьвных функций, стремяшихся к нулю на бесконечности [53], [224].

Согласно [69], описание компактных и слабо компактных операторов из банахова пространства $X$ в пространство числовых почти периодических функций $A P(S)$ на топологической полугруппе $S$ непосредственно следует из общих теорем линейного анализа. Это приводит к описанию компактных и слабо компактных мультипликаторов на пространстве $A P(S)$ в терминах равномерной непрерьвности отображения $s \mapsto \mu_{s}$, где $\mu_{s}$ - единственная векторная мера, соответствуюшая сдвинутому оператору $T_{s}\left(T_{s}(x)=(T x)_{s}\right)$. В [62] изучена связь между (слабой) почти периодичностью элементов образа оператора, сплетаюшего два банаховых представления групы, и (слабой) почти периодичностью самого сплетаюшего оператора ("мультипликатоpa") [64].

Некомпактная локально компактная група $G$ называется минимально слабо почmu периодической, если любая слабо почти периодическая функция на $G$ может быть представлена в виде суммы почти периодической функции и функции, стремящейся к нулю на бесконечности. В [55] показано, что группа движений $n$-мерного евклидова пространства и група $S L(2, \mathbb{R})$ являются минимально слабо почти периодическими, а некомпактная локально компактная связная разрешимая группа $G$ является минимально слабо почти периодической тогда и только тогда, когда факторгруппа групшы $G$ по наибольшему компактному нормальному делителю изоморфна группе движений плоскости.

В [192] введена так назьваемая представимая компактификация полутопологических полугруп (полугруппа назьвается представимой, если она имеет достаточно много равномерно ограниченных представлений в конечномерных гильбертовых пространствах) и отмечены обшие аппроксимационные свойства, связанные с этим понятием.

9.2. Приложения слабо почти периодических функций и представлений к теории чисел.

Почти периодические функции имеют теоретико-числовые приложения с момента введения их в обиход (см., например, [81], [214]). Приведем два более современных приложения.

В [87] введена специальная слабо почти периодическая функция, с помошью которой получено следуюшее обобщение теоремы Боля-Серпинского-Вейля о равномерном распределении $\bmod 1$. Если $\lambda_{1}, \ldots, \lambda_{n} \in(0,1), a_{1}, \ldots, a_{n}$ и $\alpha_{1}, \ldots, \alpha_{n}$ веществен- 
ны и числа $1, \alpha_{1}, \ldots, \alpha_{n}$ рационально независимы, то множество $P$ всех целых чисел $n$, удовлетворяющих условию $a_{i} \leqslant \alpha_{i} n \leqslant a_{i}+\lambda_{i} \bmod 1$ при $1 \leqslant i \leqslant n$, имеет слабо почти периодическую характеристическую функцию $1_{P}$ (на аддитивной группе $\mathbb{Z}$ целых чисел) со средним значением

$$
M\left(1_{P}\right)=\lim _{m \rightarrow \infty} m^{-1} \sum_{1}^{m} 1_{P}(k)=\lambda_{1} \cdots \lambda_{n}>0,
$$

так что множество $P$ бесконечно. Указано обобщение на случай $\mathbb{Z}^{m}$. Доказательство использует слабую почти периодичность функции $1_{P}$ при $P=\{n \in \mathbb{Z}: f(n) \leqslant \beta\}$ за исключением не более чем счетного множества значений $\beta$, если $f: \mathbb{Z} \rightarrow \mathbb{R}$ слабо почти периодична.

Кронекеров признак рациональности состоит в том, что арифметическая функция $g$ рекуррентна тогда и только тогда, когда лиш конечное число определителей Ганкеля

$$
\operatorname{det}\left(\begin{array}{cccc}
g(1) & g(2) & \cdots & g(m) \\
g(2) & g(3) & \cdots & g(m+1) \\
\cdots & \cdots & \cdots & \cdots \\
g(m) & g(m+1) & \cdots & g(2 m-1)
\end{array}\right)
$$

отлично от нуля. С помощью этого критерия в [146] показано, что следуюшие условия эквивалентны для любой рекуррентной последовательности $g$ (отвечающей многочлену $f(z)$, множество нулей которого мы обозначим через $A$ ).

(a) $g(n)=o(n)$.

(b) $|\alpha| \leqslant 1$ для любого $\alpha \in A$ и $f^{\prime}(\alpha) \neq 0$ для любого $\alpha$, удовлетворяюшего условию $|\alpha|=1$.

(c) $g(n)=O(1)$.

(d) Среднее значение $M(g)$ сушествует.

Отсюда следует, что если $g$ - такая 1-почти-периодическая арифметическая функция, что среднее значение функщии $n \mapsto g(n) \cdot e^{2 \pi i n \theta}$ отлично от нуля для бесконечного множества значений $\theta \in \mathbb{R} / \mathbb{Z}$, то последовательность $g$ не рекуррентна. Эти результаты развиты далее в [39].

Применения аналога "неравномерного" усреднения наполугруппе натуральных чисел к изучению свойств арифметических функций рассматривались в [113].

\section{3. Приложения к гармоническому анализу.}

Среди многочисленных прямых и косвенных приложений почти периодических функций в задачах гармонического анализа мы выделим несколько вопросов: изучение введенного и изученного в [106], [108], [109] подкласса “строго" слабо почти периодических функций, результат о структуре функций, в известном смысле двойственных почти периодическим функциям [138], аналог теоремы Бора об аргументе почти периодической функции [91], [210] и эргодические теоремы в гармоническом анализе [31], [68], [184].

Пусть $\left\{x_{n}\right\}$ - последовательность элементов $\mathbb{R} / \mathbb{Z}$. Согласно критерию Германа Вейля, последовательность $\left\{x_{n}\right\}$ равномерно распределена (р.p.) в $\mathbb{R} / \mathbb{Z}$ тогда и только тогда, когда для любой функции $f \in \mathrm{C}(\mathbb{R} / \mathbb{Z})$ выполняется равенство

$$
\mu(f) \stackrel{\text { def }}{=} \int_{\mathbb{R} / \mathbb{Z}} f(x) d x=\lim _{N \rightarrow \infty} N^{-1} \sum_{1}^{N} f\left(x_{n}\right) .
$$


Можно заменить факторгрупу $\mathbb{R} / \mathbb{Z}$ другой групой $G$, алгебру непрерьвных функций $\mathrm{CB}(G)$ - другим функциональным пространством $\mathscr{M}$, а $\mu$ - некоторьм линейньм функционалом на $\mathscr{M}$ и использовать критерий Вейля как определение последовательностей $\left\{x_{n}\right\}$, p.p. относительно $(\mathscr{M}, m)$. Если $\mathscr{B}(G)$ - пространство почти периодических функций Бора на $G$ и $m$ - однозначно определенное среднее, а $G$ локально компактна, то можно указать условия на групшу $G$, достаточные для существования последовательности, р.р. относительно $\mathscr{B}(G)$. В [106] рассматривается локально компактная группа $G$ с $\mathscr{M}=W A P(G)$ и однозначно определенньм средним $m$. Доказано, что если $G$ сепарабельна, то существует последовательность $\left\{x_{n}\right\}$, р.р. относительно $(\mathscr{W}(G), m)$. Доказательство использует эргодическую теорему фон Неймана и свойства пределов последовательностей в метризуемых локально выпуклых пространстBax.

Пусть $G$ - локально компактная топологическая групп, $\mathrm{CB}(G)-C^{*}$-алгебра комплекснозначных ограниченных непрерывных функций на $G, W A P(G)$ - пространство слабо почти периодических элементов пространства $\mathrm{CB}(G)$. Пусть $M(f)$ - единственное среднее значение функции $f$ для любой $f \in W A P(G)$. Напомним, что последовательность $\left(x_{n}\right)_{n \in \mathbb{N}}$ назьвается отлично распределенной (о.р.) относительно подмножества $W^{\prime}$ пространства $W A P(G)$, если

$$
n^{-1} \sum_{i=k+1}^{k+n} f\left(x_{i}\right) \rightarrow M(f) \text { для всех } f \in W^{\prime} \text { равномерно по } k \in \mathbb{N} \text {. }
$$

Пусть $W S(G)$ - семейство таких функций $f \in W A P(G)$, что множество $\left\{f_{g}^{h} \mid\right.$ $g, h \in G\}$ слабо относительно компактно в $\mathrm{CB}(G)$, где $f_{g}^{h}$ обозначает функцию $x \mapsto f(g x h)$ на групе $G$ со значениями в $\mathbb{C}$. В предположении, что группа $G$ сепарабельна, в [106] явно строится такая последовательность $\left\{x_{n}\right\}$, что

$$
n^{-1} \sum_{i=k+1}^{k+n} f\left(g x_{i} h\right) \rightarrow M(f)
$$

равномерно по $k \in \mathbb{N}$ и $g, h \in G$ для всех функций $f \in W S(G)$. Показано, что семейство $C_{0}(G)$ всех функций $f \in \mathrm{CB}(G)$, стремяшихся к нулю на бесконечности, содержится в $W S(G)$ тогда и только тогда, когда левая и правая равномерные структуры на $G$ совпадают. В [108], [109] показано, что $W S(G)=W(G)$ по крайней мере в двух важных частных случаях: когда групша $G$ - $[\mathrm{FIR}]$ группа и когда групша $G$ почти связна и левая и правая равномерные структуры на $G$ совпадают.

Что касается второй из упомянутых выше задач, то функции, в известном смысле двойственные к почти периодическим, изучались в [138], где рассматривались ограниченные измеримые функции на локально компактной абелевой групш $G$ с группой характеров $\widehat{G}$ и было установлено, что если пространство $L^{\infty}(G)$ содержит хотя бы одну функцию $f \neq 0$, для которой орбита под действием поточечных умножений $O(f)=$ $\{\chi \cdot f \mid \chi \in \widehat{G}\}$ относительно слабо компактна в $L^{\infty}(G)$, то $G$ дискретна, а если $G$ дискретна, то орбита $O(f)$ относительно слабо компактна в $L^{\infty}(G)$ (слабо или сильно) тогда и только тогда, когда $f$ стремится к нулю на бесконечности.

Обобщение теоремы Бора [46] имеет следующий вид. Пусть $A$ - коммутативная банахова алгебра с единичным элементом, $\mathscr{E}(A)$ - группа единищ (т.е. обратимых элементов [92]) алгебры $A$ и $\operatorname{Aut}(A)$ - группа непрерывных автоморфизмов алгебры $A$, 
снабженная сильной операторной топологией. Пусть $G$ - связная компактная группа и $T: G \rightarrow \operatorname{Aut}(A)$ - непрерьвньй гомоморфизм. Элемент $\chi \in \mathscr{E}(A)$ назьвается квазисобственным, если сушествует такой непрерьвньй гомоморфизм $t: G \rightarrow \mathscr{E}(A)$, что $T(g) \chi=\chi t(g)$ для всех $g \in G$. В [91] доказано, что для любого $f \in \mathscr{E}(A)$ сушествует такой квазисобственньй элемент $\chi \in A$ и такой элемент $\varphi \in A$, что $f=\chi \exp \varphi$. Следствием этого результата является аналог теоремы Бора об аргументе для не обращающихся в нуль почти периодических функщий Левитана на связной локально компактной абелевой групше [91].

В [31] для локально компактной абелевой групшы $G$ построено пространство $C$ функций, включающее непрерьвные почти периодические функции и (не обязательно непрерьвные) положительно определенные функции на $G$, для которого доказан аналог эргодической теоремы в предположении, что данная сеть $\left\{L_{\alpha} \mid \alpha \in A\right\}$ положительных равномерно ограниченных линейных преобразований равномерного пространства функций $C$ сходится на характерах группы $G$ к нулю, за исключением единичного характера, на котором эта сеть сходится к единице. Поскольку непрерьвные почти периодические функции равномерно порождены линейными комбинациями непрерьвных характеров, утверждение чрезвычайно близко к статистической эргодической теореме для дискретных груп. Более тонкие результаты связаны с предположениями типа измеримости положительно определенных функций. Например, как показано в [68], если $\pi$ - представление топологической группы $G$ в строго вьпуклом банаховом пространстве, если $\pi$-образ окрестности единичного элемента $e$ в $G$ имеет слабо относительно компактные орбиты в $X$ и если

$$
\lim _{g \rightarrow e}\|\Pi(g)\|=1
$$

то пространство $X$ разлагается в прямую сумму замкнутых подпространств $X_{c}$ и $X_{0}$, где ограничение представления $\pi$ на $X_{c}$ слабо непрерьвно, а замкнутая вьпуклая оболочка $\pi$-образа любой окрестности единичного элемента $e$ в $G$ содержит нулевой элемент пространства $X$. Относительньй вариант теоремы о разложении, в котором речь идет о положительно определенных функциях на локально компактной группе, почти периодических (соответственно усреднимых к нулю) относительно естественного действия замкнутого нормального делителя, получен в [184].

9.4. Приложения к устойчивости представлений абелевых полугрупп и к спектральной теории.

Пусть $G$ - $\sigma$-компактная локально компактная абелева группа, $X$ - локально выпуклое, секвенциально слабо полное топологическое векторное пространство с двойственностью $\langle X, Y\rangle$. Пусть $T: G \rightarrow \operatorname{Aut}(X)$ - представление группы $G$ автоморфизмами пространства $X$. Ненулевой вектор $x \in X$ называется собственным вектором представления $T$, если сушествует такой характер $\chi$ группы $G$, что $T(g) x=\chi(g) x$ для всех $g \in G$. В [126] указаны условия существования полного семейства собственных векторов представления $T$ в $X$. В частности, такое семейство сушествует, если $T$ - почти периодическое представление, и можно использовать условия почти периодичности скалярно почти периодических представлений. Указаны приложения теории почти периодических функций к изучению условий полноты системы собственных векторов обобшенно нормальных операторов. 


\section{5. Теоремы о неподвижной точке и инвариантные подпространства.}

В [131] изучались конечномерные инвариантные подпространства полугрупп линейных операторов в локально вьпуклых пространствах. Представление $\mathscr{S}=\left\{T_{s}\right.$ : $s \in S\}$ полутопологической полугрупш $S$ линейными операторами в отделимом локально выпуклом пространстве $E$ назьвается раздельно непрерывным, если отображение $(s, x) \mapsto T_{s} x$ произведения $S \times E$ в $E$ непрерьвно по каждому переменному. Оно называется совместно непрерывныл на множестве $K \subset E$, если оно непрерьвно на произведении $S \times K$. Пусть $n$ - натуральное число. Рассмотрим множество всех таких раздельно непрерьвных представлений $\mathscr{S}$ топологической полугруппы $S$ в пространстве $E$, которые непрерьвны (непрерывны по совокупности переменных) на компактных выпуклых подмножествах пространства $E$. Пусть $\mathscr{L}_{n}(X)$ - семейство всех $n$-мерных подпространств пространства $E$, которые содержатся в данном подмножестве $X$ пространства $E$. Говорят, что полугрупа $S$ имеет свойство $P(n)$, если для любого такого представления $\mathscr{S}$ и любого такого подмножества $X \subset E$, что существует замкнутое $\mathscr{S}$-инвариантное подпространство $H$ в $E$ коразмерности $n$, для которого множество $x+H \cap X$ компактно и выпукло для любого $x \in E$, из условия, что $\mathscr{L}_{n}(X)$ непусто и $\mathscr{S}$-инвариантно, следует, что существует такое подпространство $L_{0} \in \mathscr{L}_{n}(X)$, что $T_{s}\left(L_{0}\right)=L_{0}$ для каждого $s \in S$. Доказьвается, что если $S$ левоаменабельна (т.е. пространство $L U C(S)$ леворавномерно непрерывных функций на $S$ имеет левоинвариантное среднее), то $S$ имеет свойство $P(n)$ для всех $n$. Обратно, если $S$ имеет свойство $P(1)$, то $S$ левоаменабельна (и поэтому имеет свойство $P(n)$ для всех $n$ ). Этот результат является аналогом и обобщением утверждения Фань Сюя [83]. Аналогичные результаты получены для почти периодических функций (элементов пространства $A P(S)$ ) и представлений, равностепенно непрерьвных на компактных вьпуклых подмножествах, а также для слабо почти периодических функций (элементов пространства $W A P(S))$ и представлений, квазиравностепенно непрерывных на компактных выпуклых подмножествах.

\section{$\S$ 10. Заключительные замечания}

Теория числовых почти периодических функций на группах связана с проблемой представимости топологических груп, т.е. существования семейства унитарных представлений данной групы, разделяющего точки группы, которая положительно решена для локально компактных груп теоремой Гельфанда-Райкова, и положительное решение легко распространяется на случай локально вполне ограниченных групп (информацию от этом классе можно найти, в частности, в [60]), но границы класса унитарно представимых групп неясны. Известно, что любая хаусдорфова топологическая группа имеет точное представление в банаховом пространстве $[211]^{12}$, но пространство этого представления не обязано быть ни гильбертовьм, ни даже рефлексивньм. Кроме того, имеются как отрицательные результаты, показьваюшие, что есть топологические группы, не допускаюшие унитарных представлений в гильбертовом пространстве (см. обзорную часть в [204]), так и критерии того, что топологическая група имеет достаточно много представлений в рефлексивных банаховых пространствах [198] (заметим, что в [154] показано, что топологическая группа, имеющая

\footnotetext{
${ }^{12} \mathrm{Ecли} G$ - произвольная хаусдорфова топологическая группа, то утверждение следствия 2 в [147; 1 гл. III] ошибочно, а если дискретная, то это утверждение очевидно.
} 
достаточно много представлений в рефлексивных банаховых пространствах, может иметь недостаточно много представлений в гильбертовых пространствах), но общая картина далека от полной ясности. Последние успехи в этом направлении связаны с описанием локально вполне ограниченных топологических груп, все топологически неприводимые представления которых в гильбертовом пространстве конечномерны, и полным описанием представимых топологических групп, размерности топологически неприводимых представлений которых в гильбертовом пространстве конечны и ограничены в совокупности [204].

\section{Приложения}

\section{Приложение 1. Полнота по фон Нейману и свойство Крейна.}

Напомним определение полноты локально вьпуклых пространств по Муру (сам Мур назьвал эту полноту “ваффельной полнотой” (wafer complete space), cp. [76; предложение 8.14.3]), или, что то же, определение свойства Крейна.

ОПРЕДЕЛЕНИЕ 12. Локально вьпуклое пространство $E$ назьвается имеющим свойство Крейна или полным по Муру [168; гл. 2], если слабо замкнутая уравновешенная выпуклая оболочка любого слабо компактного подмножества в $E$ является слабо компактньм множеством.

Классическая теорема Крейна утверждает, что любое банахово пространство имеет свойство Крейна (т.е. полно в смысле Мура). Хорошо известно (см, например, теорему 8.13.1 в [76] и IV.11.5 в [168]), что слабо замкнутая вьпуклая (уравновешенная) оболочка компактного множества в хаусдорфовом локально выпуклом пространстве $E$ компактна тогда и только тогда, когда эта оболочка полна в топологии Макки $\tau\left(E, E^{*}\right)$. В частности, любое квазиполное локально выпуклое пространство имеет свойство Крейна. ${ }^{13}$

Теория почти периодических функций в топологических (в частности, локально вьпуклых) векторных пространствах начиналась [219] с другого условия полноты, введенного фон Нейманом специально для этого случая в [220].

ОПРЕДЕЛЕНИЕ 13. Локально вьпуклое пространство $E$ называется noлнblм $n o$ фон Нейману или полным в смысле фон Неймана, если замыкание любого предкомпактного множества в $E$ компактно.

Очевидно, что наличие свойства Крейна (условие полноты по Муру) следует из условия полноты по фон Нейману в слабой топологии, но это последнее условие чрезвычайно сильно. Нетрудно заметить, что банахово пространство полно по фон Нейману в слабой топологии тогда и только тогда, когда оно рефлексивно. Другое условие на топологические векторные пространства, используемое фон Нейманом в [219], [220], состоит в существовании последовательности открытых окрестностей нуля, пересечение которых содержит только сам нулевой элемент. Как мы видим, эти два условия, высказанные фон Нейманом в [220], т.е. за пределами статьи [219] о почти периодических функциях, весьма ограничительны.

\footnotetext{
${ }^{13}$ Следует заметить, что если $B$ - замкнутая уравновешенная выпуклая оболочка слабо компактного подмножества хаусдорфова локально выпуклого пространства $E$, то $B$ слабо компактно тогда и только тогда, когда $B$ полно относительно некоторой (или, что равносильно, любой) топологии, согласованной с двойственностью между $E$ и $E^{*}$ (см. [168; гл. 2, теорема 6]).
} 


\section{Приложение 2. Интегрирование операторных функций.}

В этой части приложения мы напоминаем некоторые фольклорные факты об условиях существования и свойствах так назьваемого "слабого интеграла" от векторных и операторных функций со значениями в локально вьпуклом пространстве. Пусть $X-$ компактное хаусдорфово пространство и $\mu$ - регулярная борелевская мера на $X$.

ПРЕДЛОЖЕНИЕ 1. Пусть $X$ - компактное топологическое пространство, $\mu$ регулярная борелевская мера на $X$, а $E$-локально выпуклое пространство. Для любой слабо непрерывной функиии $f: X \rightarrow E$ формула

$$
F(x)=\int_{X} x(f(t)) d \mu(t), \quad x \in E^{*}
$$

корректно определяет линейный функционал $F$ на пространстве $E^{*}$ и если локально выпуклое пространство Е имеет свойство Крейна, то этот линейный функиионал определен әлементом $E$, т.е. существует такой әлемент у $\in E$, чтоo

$$
F(x)=x(y) \quad \text { для любьх } \quad x \in E^{*} .
$$

ДокАЗАТЕЛЬство. См. [76; теорема 8.13.1].

ОПРЕДЕЛЕНИЕ 14. В ситуации, описанной в предложении 1 , элемент $y$, определенньй формулой (13), назьвается “слабым интегралом" от функции $f$. Положим

$$
y=\int_{X} f(t) d \mu(t)
$$

Список стандартных свойств этого интеграла приведен в $[76 ;$ гл. $8, \S 14]$ и в $[147$; гл. 2].

Докажем теперь, что можно интегрировать и непрерьвные линейные операторы.

ПРЕДЛОЖЕНИЕ 2 (интегрирование линейных операторов). Пусть $E$ - локально выпуклое пространство, $F$ - пространство Макки со свойством Крейна, а $\mathscr{L}(E, F)$ - пространство непрерывных линейных операторов из $E$ в $F$. Введем в пространстве $\mathscr{L}(E, F)$ слабую операторную топологию. Пусть $X$ - компактное топологическое пространство и $\mu$-регулярная борелевская мера на $X$. Введем в пространстве $\mathrm{C}(X, \mathscr{L}(E, F))$ равномерность, определенную естественной равномерностью на $\mathscr{L}(E, F)[195 ; \mathrm{I} .1 .4]$, т.е. окружения в $\mathrm{C}(X, \mathscr{L}(E, F))$ - множества вида $O_{V}=\left\{\left(f_{1}, f_{2}\right) \in \mathrm{C}(X, \mathscr{L}(E, F)) \times \mathrm{C}(X, \mathscr{L}(E, F)) \mid\left(f_{1}(\xi), f_{2}(\xi)\right) \in V\right.$ для любых $\xi \in X\}$, где $V$ пробегает семейство всех окружений в равномерном пространстве $\mathscr{L}(E, F)$. Для любого әлемента $f \in \mathrm{C}(X, \mathscr{L}(E, F))$ и любого вектора $\xi \in E$ функиия $f_{\xi}$ на $X$, определенная формулой $f_{\xi}(x)=f(x) \xi, x \in X$, $\xi \in E$, задает непрерывную функиию на $X$ со значениями в пространстве $F$. Для любьх $f \in \mathrm{C}(X, \mathscr{L}(E))$ u $\xi \in E$ обозначим через $y(f)$ әлемент $y(f, \xi) \in F$, однозначно определенный (согласно предложению 1) формулой

$$
x(y(f, \xi))=\int_{X} x\left(f_{\xi}(t)\right) d \mu(t), \quad x \in F^{*}
$$


В этом случае существует такой слабо непрерывный линейный оператор $z_{f} \in \mathscr{L}(E, F)$, что отображсение $(f, \xi) \mapsto y(f, \xi), \quad f \in \mathrm{CB}(X, \mathscr{L}(E, F))$, $\xi \in E$, задается формулой $y(f, \xi)=z_{f} \xi, \quad f \in \mathrm{C}(X, \mathscr{L}(E, F)), \quad \xi \in E$, и отобрахсение $z: \mathrm{C}(X, \mathscr{L}(E, F)) \rightarrow \mathscr{L}(E, F)$, определенное формулой $z(f)=z_{f}$, $f \in \mathrm{C}(X, \mathscr{L}(E, F))$, непрерывно в указанных выше топологиях равномерной сходимости в слабой операторной топологии на $\mathrm{C}(X, \mathscr{L}(E, F))$ и слабой операторной топологии на $\mathscr{L}(E, F)$.

Именно ради свойства непрерьвности в предложении 2 введено предположение, что локально вьпуклое пространство $E$ есть пространство Макки. Напомним, что любое бочечное пространство является пространством Макки [195; теорема IV.3.4].

ДоКАЗАТЕЛЬСТВо ПРЕДЛОЖЕНИЯ 2. Из определения слабой операторной топологии на $\mathscr{L}(E, F)$ следует, что эта топология индуцирована системой полунорм вида $T \mapsto|f(T x)|, x \in E, f \in F^{*}$ (где $F^{*}$, как всегда, обозначает пространство, сопряженное к $F)$.

Докажем сначала, что оператор $z(f)$ непрерьвен для любой функции $f \in$ $\mathrm{C}(X, \mathscr{L}(E, F))$, если $E$ снабжено топологией Макки, а $F$ - слабой топологией. Достаточно доказать, что оператор $z$ непрерьвен в нуле. По предположению для любых $\xi \in E$ функция $t \mapsto f(t) \xi, t \in X$, непрерывна на $X$, если пространство $F$ снабжено слабой топологией. Отсюда следует, что функция $t \mapsto F(f(t) \xi)$ непрерьвна на $X$ для любых $\varphi \in F^{*}$ и $x \in E$, и, следовательно, функция $t \mapsto f(t)^{*} \varphi$ непрерьвна на $X$ по отношению к слабой топологии на пространстве $E^{*}$ для любых $\varphi \in E^{*}$, где $f(t)^{*}$ - оператор, сопряженньй к $f(t)$. Поэтому множество $f(X)^{*} \varphi$, образованное значениями функции $f(\cdot) * \varphi$ на пространстве $X$, слабо* компактно (т.е. компактно в $\sigma\left(E^{*}, E\right)$-топологии). Если ограниченная сеть $\xi_{\alpha}$ стремится к нулю в $E$ в топологии Макки (это означает, что $\xi_{\alpha}$ стремится к нулю в топологии равномерной сходимости на слабо компактных вьпуклых уравновешенных множествах в пространстве $E^{*}$ ), то отсюда следует (так как $E$ предполагалось имеюшим свойство Крейна), что $\xi_{\alpha}$ стремится к нулю в топологии равномерной сходимости на слабо* компактных множествах в $E^{*}$ и, в частности, сеть $\xi_{\alpha}$ равномерно стремится к нулю на множестве $f(X)^{*} \varphi$. Переходя к пределу в скалярных интегралах

$$
\varphi\left(z(f) \xi_{\alpha}\right)=\int_{X} \varphi\left(f(t) \xi_{\alpha}\right) d \mu(t), \quad \varphi \in F^{*}, \quad \xi_{\alpha} \in E, \quad f \in \mathrm{C}(X, \mathscr{L}(E, F))
$$

с помошю равномерной сходимости подынтегральных выражений к нулю на всем пространстве $X$, мы видим, что $\varphi\left(z(f) \xi_{\alpha}\right)$ стремится к нулю для любых $\varphi \in F^{*}$. Следовательно, функция $z(f) \xi_{\alpha}$ стремится к нулю в слабой топологии на $F$. Это доказьвает, что для любых $f \in \mathrm{C}(X, \mathscr{L}(E, F))$ линейное отображение $z(f)$ из $E$ в $F$ непрерьвно, если $E$ снабжено топологией Макки (мы будем писать $E_{\tau}$, чтобы указать, что $E$ снабжено топологией $\left.\tau\left(E, E^{*}\right)\right)$, а $F$ - слабой топологией (мы будем писать $F_{\sigma}$, чтобы указать, что $F$ снабжено топологией $\left.\sigma\left(F, F^{*}\right)\right)$. Но этого условия непрерьвности достаточно для того, чтобы сопряженньй оператор был определен на всем пространстве $F_{\sigma}^{*}$ (как непрерьвное отображение сопряженных пространств, а именно пространства $F_{\sigma}^{*}$ в $\left.E_{\tau}^{*}\right)$. По запасу элементов эти пространства совпадают с $F^{*}$ и $E^{*}$ соответственно (с точностью до топологий, которые могут быть различными) и, следовательно, сопряженное к $z(f)$ отображение переводит $F^{*}$ в $E^{*}$ для любых $f \in \mathrm{C}(X, \mathscr{L}(E, F))$. Со- 
гласно теореме IV.2.1 в [195], отсюда следует, что $z(f)$ непрерывно в слабых топологиях на $E$ и $F$. По предположению $E$ и $F$ - пространства Макки и, следовательно, линейньй оператор из $E$ в $F$ непрерьвен тогда и только тогда, когда он слабо непрерывен [195; теорема IV.7.4]. Таким образом, $z(f)$ непрерывен для любых $f \in \mathrm{C}(X, \mathscr{L}(E, F))$, что завершает доказательство первого из утверждений.

Утверждение о непрерьвности отображения $z: \mathrm{C}(X, \mathscr{L}(E, F)) \rightarrow \mathscr{L}(E, F)$ в топологиях, указанных в теореме, доказьвается аналогично. Мы опустим подробности, поскольку мы не пользуемся этой частью утверждения в основном тексте статьи.

\section{СПИСОК ЛИТЕРАТУРЫ}

[1] А. Ю. Александров. О существовании асимптотически рекуррентных движений динамических систем // Дифференц. уравнения. 1994. Т. 30. № 4. С. 720-722.

[2] L. Amerio. Almost-periodic functions in Banach spaces // Mat.-Fys. Medd. Danske Vid. Selsk. 1989. V. 42. №3. P. 25-33.

[3] M. E. Andersson. The counterpart for Hankel-Schur multipliers of Eberlein's decomposition // Research Reports in Mathematics № 2. Stockholm: Stockholm University, Department of Mathematics, 2002.

[4] K. T. Andrews, J. J. Uhl, Jr. Weak compactness in $L^{\infty}(\mu, X) / /$ Indiana Univ. Math. J. 1981. V. 30. №6. P. 907-915.

[5] W. Arendt, C. J.K. Batty. Tauberian theorems and stability of one-parameter semigroups // Trans. Amer. Math. Soc. 1988. V. 306. № 2. P. 837-852.

[6] W. Arendt, S. Schweiker. Discrete spectrum and almost periodicity // Taiwanese J. Math. 1999. V. 3. № 4. P. 475-490.

[7] J. Arias de Reyna, J. Diestel, V. Lomonosov, L. Rodríguez-Piazza. Some observations about the space of weakly continuous functions from a compact space into a Banach space // Quaest. Math. 1992. V. 15. №4. P. 415-425.

[8] W. Arveson. The harmonic analysis of automorphism groups // Proc. Sympos. Pure Math. 1982. V. 38. P. 199-269.

[9] Banach, S.. Théorie des opérations linéaires. Warszawa: Subwencji Funduszu Narodowej, 1932.

[10] Bart, H., Goldberg, S.. Characterizations of almost periodic strongly continuous groups and semigroups // Math. Ann. 1978. V. 236. № 2. P. 105-116.

[11] Б.Р. Басит. Связь между почти периодическими функциями Левитана и почти автоморфными функциями // Вестник МГУ. Сер. І матем., мех. 1971. №4. С. 11-15.

[12] Б.Р. Басит. Обобщение двух теорем М.И. Кадеца о неопределенном интеграле абстрактных почти периодических функций // Матем. заметки. 1971. Т. 9. С. 311-321.

[13] B. Basit. Note on a theorem of Levitan for the integral of almost periodic functions // Rend. Istit. Mat. Univ. Trieste. 1973. V. 5. P. 9-14.

[14] B. Basit. Les fonctions abstraites presque automorphiques et presque périodiques au sens de Levitan, et leurs differences // Bull. Sci. Math. (2). 1977. V. 101. №2. P. 131-148.

[15] B. Basit. On the indefinite integrals of abstract functions // An. Ştiinţ. Univ. Al. I. Cuza Iaşi Sect. I a Mat. 1983. V. 29. № 3, suppl. P. 49-54.

[16] B. Basit. Some problems concerning different types of vector valued almost periodic functions // Dissertationes Math. (Rozprawy Mat.) 1995. V. 338.

[17] B. Basit. Harmonic analysis and asymptotic behavior of solutions to the abstract Cauchy problem // Semigroup Forum. 1997. V. 54. №1. P. 58-74.

[18] B. Basit, M. Emam. Differences of functions in locally convex spaces and applications to almost periodic and almost automorphic functions // Ann. Polon. Math. 1983. V. 41. № 3. P. 193-201.

[19] B. Basit, H. Günzler. Asymptotic behavior of solutions of systems of neutral and convolution equations // J. Differential Equations. 1998. V. 149. №1. P. 115-142. 
[20] B. Basit, H. Günzler. Generalized Esclangon-Landau results and applications to linear difference-differential systems in Banach spaces // J. Differ. Equations Appl. 2004. V. 10. № 11. P. 1005-1023.

[21] B. Basit, H. Günzler. A difference property for perturbations of vector-valued Levitan almost periodic functions and analogues // Russian J. Math. Phys. 2005. V. 12. №4 (to appear).

[22] B. Basit, A. J. Pryde. Ergodicity and differences of functions on semigroups // J. Austral. Math. Soc. Ser. A. 1998. V. 64. № 2. P. 253-265.

[23] B. Basit, A. J. Pryde. Ergodicity and stability of orbits of unbounded semigroup representations // J. Austral. Math. Soc. 2004. V. 77. № 2. P. 209-232.

[24] B. Basit, A. J. Pryde. Asymptotic behavior of orbits of $C_{0}$-semigroups and solutions of linear and semilinear abstract differential equations // Russian J. Math. Phys. 2005. V. 12. №4 (to appear).

[25] B. Basit, C. Zhang. New almost periodic type functions and solutions of differential equations // Canad. J. Math. 1996. V. 48. №6. P. 1138-1153.

[26] Б. Р. Басит, В.В.Жиков. Почти периодические решения интегро-дифференциалшых уравнений в пространстве Банаха // Вестник МГУ. Сер. Іматем., мех. 1971. № 2. С. 29-33.

[27] А. Г. Баскаков. Спектральные признаки почти периодичности решений функциональных уравнений // Матем. заметки. 1978. Т. 24. № 2. С. 195-206.

[28] А. Г. Баскаков. Гармонический анализ косинуса и показательной операторной функции // Матем. сб. 1984. Т. 124 (166). №1. С. 68-95.

[29] J. Batt, M.V. Deshpande. Weak convergence and weak compactness in the space of almost periodic functions on the real line // Proc. Indian Acad. Sci. Math. Sci. 1996. V. 106. № 1. P. 1-11.

[30] A. Belanger, B. E. Forrest. Geometric properties of some subspaces of $V N(G) / /$ Proc. Amer. Math. Soc. 1994. V. 122. №1. P. 131-133.

[31] J.-M. Belley. An ergodic theorem in harmonic analysis // C. R. Math. Rep. Acad. Sci. Canada. 1981. V. 3. №4. P. 203-208.

[32] J. F. Berglund. On extending almost periodic functions // Pacific J. Math. 1970. V. 33. P. 281-289.

[33] J. F. Berglund. Dissipative weakly almost periodic functions // Mat.-Fys. Medd. Danske Vid. Selsk. 1989. V. 42. № 3. P. 77-80.

[34] J. F. Berglund, N. Hindman. Filters and the weak almost periodic compactification of a discrete semigroup // Trans. Amer. Math. Soc. 1984. V. 284. № 1. P. 1-38.

[35] J. F. Berglund, K. H. Hofmann. Compact Semitopological Semigroups and Weakly Almost Periodic Functions. Berlin: Springer-Verlag, 1967. (Lecture Notes in Math. V. 42.)

[36] J. F. Berglund, H.D. Junghenn, P. Milnes. Compact Right Topological Semigroups and Generalizations of Almost Periodicity. Berlin: Springer-Verlag, 1978. (Lecture Notes in Math. V. 663.)

[37] J. F. Berglund, H.D. Junghenn, P. Milnes. Analysis on Semigroups. New York: Wiley, 1989.

[38] A.S. Besicovitch. Almost Periodic Functions. New York: Dover, 1955.

[39] J.-P. Bézivin. Sur les suites presque-périodiques // Arch. Math. (Basel). 1998. V. 70. №6. P. 447-454.

[40] J. Blot. Le théorème de Markov-Kakutani et la presque-périodicité // Fixed Point Theory and Applications (Marseille, 1989). Harlow: Longman, 1991. P. 45-56. (Pitman Res. Notes Math. Ser. V. 252.)

[41] S. Bochner. A new approach to almost periodicity // Proc. Natl. Acad. Sci. USA. 1962. V. 48. P. 2039-2043.

[42] S. Bochner. General almost automorphy // Proc. Natl. Acad. Sci. USA. 1975. V. 72. № 10. P. 3815-3818.

[43] S. Bochner, J. von Neumann. Almost periodic functions in groups. II // Trans. Amer. Math. Soc. 1935. V. 37. P. 21-50; рус. пер.: Почти периодические функции на группах. II // Дж. фон Нейман. Избранные труды. Т. І. М.: Наука, 1987. С. 146-170. 
[44] Н.Н. Боголюбов. О некоторых статистических методах в математической физике. Львов: Изд-во АН УССР, 1945.

[45] P. Bohl. Über eine Differentialgleichnung der Störungstheorie // Crelles J. 1906. V. 131. P. 268-321.

[46] H. Bohr. Collected Mathematical Works. V. II, III. København: Dansk Matematisk Forening, 1952 .

[47] B. Bordbar, J. Pym. The weakly almost periodic compactification of a direct sum of finite groups // Math. Proc. Cambridge Philos. Soc. 1998. V. 124. № 3. P. 421-449.

[48] B. Bordbar, J. Pym. The set of idempotents in the weakly almost periodic compactification of the integers is not closed // Trans. Amer. Math. Soc. 2000. V. 352. № 2. P. 823-842.

[49] J. Bourgain, M. Talagrand. Compacité extrêmale // Proc. Amer. Math. Soc. 1980. V. 80. №1. P. $68-70$.

[50] G. Brown, I. Glicksberg, E. Hewitt. Indicator functions with large Fourier transforms // Pacific J. Math. 1983. V. 105. №1. P. 11-20.

[51] R. B. Burckel. Weakly Almost Periodic Functions on Semigroups. New York: Gordon and Breach, 1970.

[52] R. Chill. Tauberian theorems for vector-valued Fourier and Laplace transforms // Studia Math. 1998. V. 128. №1. P. 55-69.

[53] C. Chou. Weakly almost periodic functions and almost convergent functions on a group // Trans. Amer. Math. Soc. 1975. V. 206. P. 175-200.

[54] C. Chou. Uniform closures of Fourier-Stieltjes algebras // Proc. Amer. Math. Soc. 1979. V. 77. № 1. P. 99-102.

[55] C. Chou. Minimally weakly almost periodic groups // J. Funct. Anal. 1980. V. 36. №1. P. $1-17$.

[56] C. Chou. Weakly almost periodic functions and Fourier-Stieltjes algebras of locally compact groups // Trans. Amer. Math. Soc. 1982. V. 274. № 1. P. 141-157.

[57] C. Chou. Weakly almost periodic functions and thin sets in discrete groups // Trans. Amer. Math. Soc. 1990. V. 321. № 1. P. 333-346.

[58] C. Chou. Almost periodic operators in VN $(G) / /$ Trans. Amer. Math. Soc. 1990. V. 317. №1. P. 229-253.

[59] C. Chou, A.T.-M. Lau, J. Rosenblatt. Approximation of compact operators by sums of translations // Illinois J. Math. 1985. V. 29. №2. P. 340-350.

[60] W.W. Comfort. Topological groups // Handbook of Set-theoretic Topology. Amsterdam: North-Holland, 1984. P. 1143-1263.

[61] M. Cowling, P. Rodway. Restrictions of certain function spaces to closed subgroups of locally compact groups // Pacific J. Math. 1979. V. 80. №1. P. 91-104.

[62] G. Crombez. Compactness and almost periodicity of multipliers // Canad. Math. Bull. 1983. V. 26. №1. P. 58-62.

[63] G. Crombez. Subspaces of $L_{\infty}(G)$ with unique topological left invariant mean // Czechoslovak Math. J. 1984. V. 34 (109). №2. P. 178-182.

[64] G. Crombez, W. Govaerts. Strongly and weakly almost periodic multipliers from $L_{1}(G)$ to $L_{\infty}(G) / /$ Bull. Soc. Math. Belg. Ser. B. 1980. V. 32. № 2. P. 179-188.

[65] C. Datry, G. Muraz. Analyse harmonique dans les modules de Banach, II: presque-périodicité et ergodicité // Bull. Sci. Math. 1996. V. 120. №6. P. 493-536.

[66] М.М.Дэй. Нормированные линейные пространства. М.: ИЛ, 1961.

[67] K. de Leeuw, I. Glicksberg. Applications of almost periodic compactifications // Acta Math. 1961. V. 105. P. 63-97.

[68] K. de Leeuw, I. Glicksberg. The decomposition of certain group representations // J. Analyse Math. 1965. V. 15. P. 135-192.

[69] M.V. Deshpande, M. N. Parekh. Compact operators on a Banach space into the space of almost periodic functions // Proc. Indian Acad. Sci. Math. Sci. 1990. V. 100. № 1. P. 25-36. 
[70] J. Diestel. A survey of results related to the Dunford-Pettis property // Proceedings of the Conference on Integration, Topology, and Geometry in Linear Spaces (Univ. North Carolina, Chapel Hill, N.C., 1979). Providence, RI: Amer. Math. Soc., 1980. P. 15-60. (Contemp. Math. V. 2.)

[71] J. Diestel, J. J. Uhl, Jr. Vector Measures. Providence, RI: American Mathematical Society, 1977. (Mathematical Surveys. V. 15.)

[72] D. N. Dikranjan, I. R. Prodanov, L. N. Stoyanov. Topological Groups. Characters, Dualities and Minimal Group Topologies. New York: Dekker, 1990. (Monogr. Textbooks Pure Appl. Math. V. 130.)

[73] L. Drewnowski. On subspaces of locally convex spaces with unconditional Schauder bases // Colloq. Math. 1987. V. 52. №2. P. 239-245.

[74] W. F. Eber lein. Abstract ergodic theorems and weak almost periodic functions // Trans. Amer. Math. Soc. 1949. V. 67. P. 217-240.

[75] W.F. Eberlein. The point spectrum of weakly almost periodic functions // Michigan Math. J. 1955-56. V. 3. P. 137-139.

[76] Р. Эдвардс. Функциональньй анализ. М.: Мир, 1969.

[77] R. Ellis. Locally compact transformation groups // Duke Math. J. 1957. V. 24. P. 119-125.

[78] R. Ellis. Lectures on topological dynamics. New York: Benjamin, 1969.

[79] R. Ellis. The enveloping semigroup of projective flows // Ergodic Theory Dynam. Systems. 1993. V. 13. №4. P. 635-660.

[80] Р. Энгелькинг. Общая топология. М.: Мир, 1986.

[81] P. Erdős, M. Kac, E. R. van Kampen, A. Wintner. Ramanujan sums and almost periodic functions // Studia Math. 1940. V. 9. P. 43-53.

[82] R. Eymard. L'algèbre de Fourier d'un groupe localement compact // Bull. Soc. Math. France. 1964. V. 92. P. 181-236.

[83] Ку Fan [Сюй Фань]. Invariant subspaces for a semigroup of linear operators // Nederl. Akad. Wetensch. Proc. Ser. A. V. $68=$ Indag. Math. 1965. V. 27. P. 447-451.

[84] S. Ferri, D.Strauss. A note on the $W A P$-compactification and the $L U C$-compactification of a topological group // Semigroup Forum. 2004. V. 69. №1. P. 87-101.

[85] A. M.Fink, G.H. Meisters. On $\Delta-m$ sets, almost periodic functions and group topologies // Rocky Mountain J. Math. 1972. V. 2. № 2. P. 225-233.

[86] E. Følner. On groups with full Banach mean value // Math. Scand. 1946. V. 3. P. 243-254.

[87] E. Følner. Some special $W$-almost periodic sets on the integers // Math. Scand. 1983. V. 53. № 2. P. 185-192.

[88] J. Gil de Lamadrid, L. N. Argabright. Almost periodic measures // Mem. Amer. Math. Soc. 1990. V. 85. № 428.

[89] T. A. Gillespie, T. T. West. Weakly compact groups of operators // Proc. Amer. Math. Soc. 1975. V. 49. P. 78-82.

[90] I. Glicksberg. Uniform boundedness for groups // Canad. J. Math. 1962. V. 14. P. 269-276.

[91] S. Goldberg, P. Irwin. Weakly almost periodic vector-valued functions // Dissertationes Math. (Rozprawy Mat.). 1979. V. 157.

[92] Е.А. Горин. Функционально-алгебраический вариант теоремы Бора-ван Кампена // Матем. сб. 1970. Т. 82 (124). №2. С. 260-272.

[93] Е. А. Горин. Некоторые замечания о теореме Гельфанда о группе обратимых элементов банаховой алгебры // Функц. анализ и его прил. 1978. Т. 12. №1. С. 70-71.

[94] H. Gottschalk, G. A. Hedlund. Topological Dynamics. Providence, RI: Amer. Math. Soc., 1955.

[95] C. C. Graham, T. Ramsey. Three results on I-sets // Colloq. Math. 1987. V. 51. P. $141-147$.

[96] E. E. Granirer. The Schur property and the WRNP for submodules of the dual of the Fourier algebra $A(G)$ // C. R. Math. Acad. Sci. Soc. R. Can. 1997. V. 19. № 1. P. 15-20.

[97] Ф. Гринлиф. Инвариантные средние на топологических группах и их приложения. М.: Мир, 1973. 
[98] W. Greve. Dini's theorem for almost periodic functions // J. Austral. Math. Soc. 1961/1962. V. 2. P. 143-146.

[99] С.А. Григорян. Обобщенные аналитические функции // УМН. 1994. Т. 49. №2. C. $3-42$.

[100] A. Grothendieck. Critères de compacité dans les espaces fonctionnels généraux // Amer. J. Math. 1952. V. 74. P. 168-186.

[101] H. Günzler. Integration of almost periodic functions // Math. Z. 1967. V. 102. P. 253-287.

[102] H. Günzler. Vector valued functions on semigroups with almost periodic differences // Atti Accad. Naz. Lincei Rend. Cl. Sci. Fis. Mat. Natur. (8). 1967. V. 42. P. 775-781.

[103] H. Günzler. Means over countable semigroups and almost-periodicity in $l^{1} / / \mathrm{J}$. Reine Angew. Math. 1968. V. 232. P. 194-206.

[104] H. Günzler. On the countability of the spectrum of weakly almost periodic functions // Proceedings of the International Conference on Partial Differential Equations dedicated to Luigi Amerio on his 70th birthday (Milan/Como, 1982). Milan: Univ. Studi Milano, 1985. P. 109-147. (Rend. Sem. Mat. Fis. Milano. V. 52.)

[105] A. E. Hamza, G. L. Muraz. Spectral criteria of abstract functions; integral and difference problems // Acta Math. Vietnam. 1998. V. 23. № 1. P. 171-184.

[106] G. Hansel, J.-P. Troallic. Suites uniformément distribuées et fonctions faiblement presque-périodiques // Bull. Soc. Math. France. 1980. V. 108. № 2. P. 207-212.

[107] G. Hansel, J.-P. Troallic. Points de continuité à gauche d'une action de semi-groupe // Semigroup Forum. 1983. V. 26. №3-4. P. 205-214.

[108] G. Hansel, J.-P. Troallic. On a class of weakly almost periodic mappings // Semigroup Forum. 1990. V. 41. №3. P. 357-372.

[109] G. Hansel, J.-P. Troallic. Moore groups have the WS-property // Semigroup Forum. 1993. V. 46. № 2. P. 146-151.

[110] D. Helmer. Continuity of semigroup actions // Semigroup Forum. 1981. V. 23.№ 2 . P. $153-188$.

[111] S. Hernández, S. Macario. Invariance of compactness for the Bohr topology // Topology Appl. 2001. V. 111. № 2. P. 161-173.

[112] Э. Хьюитт, К. А. Росс. Абстрактный гармонический анализ. Т. І. М.: Наука, 1975.

[113] K.-H. Indlekofer. On some spaces of arithmetical functions. I // Anal. Math. 1992. V. 18. №3. P. 203-221.

[114] K. Iséki. Vector-space valued functions on semi-groups. I, II, III // Proc. Japan Acad. 1955. V. 31. P. 16-19, 152-155, 699-701.

[115] K. Jacobs. Ergodentheorie und fastperiodische Funktionen auf Halbgruppen // Math. Z. 1956. V. 64. P. 298-338.

[116] K. Jacobs. Fastperiodizitätseigenschaften allgemeiner Halbgruppen in Banach-Räumen // Math. Z. 1957. V. 67. P. 83-92.

[117] B. E. Johnson. Cohomology of Banach Algebras. Providence, RI: Amer. Math. Soc., 1972. (Mem. Amer. Math. Soc. V. 127.)

[118] H. D. Junghenn. Weakly almost periodic representations of semigroups by Markov operators // Semigroup Forum. 1987. V. 35. № 2. P. 195-205.

[119] H. D. Junghenn. Direct sums of spaces of functions on semigroups // Semigroup Forum. 1994. V. 49. №1. P. 115-123.

[120] H.D. Junghenn. Operator semigroup compactifications // Trans. Amer. Math. Soc. 1996. V. 348. № 3. P. 1051-1073.

[121] H.D. Junghenn, P. Milnes. Distal compactifications of group extensions // Rocky Mountain J. Math. 1999. V. 29. № 1. P. 209-227.

[122] М.И. Кадец. Об интегрировании почти периодических функций со значениями в пространстве Банаха // Функц. анализ и его прил. 1969. Т. 3. № 3. С. 71-74.

[123] М.И. Кадец. О связи между сильной и скалярной почти периодичностью банаховых представлений группы вещественных чисел // Функц. анализ и его прил. 1995. Т. 29. № 4. C. $75-77$. 
[124] М. И. Кадец, К. Д. Кюрстен. Счетность спектра слабо почти периодических функций со значениями в банаховом пространстве // Теория функций, функц. анализ и прил. 1980. T. 33. С. $45-49$.

[125] М. И. Кадец, Ю. И. Любич. О связи между различными формами почти периодичности представлений групп // Теория функций, функц. анализ и прил. 1990. Т. 53. С. 3-5.

[126] М.И. Караханян. Условия полноты системы собственных векторов обобщенно нормального оператора // Ереван. гос. ун-т. Учен. зап. Естеств. науки. 1988/1989. № 3. С. 3-8.

[127] T. Kayano. Almost periodic functions on topological semigroups // Mem. Fac. Sci. Shimane Univ. 1979. V. 13. P. 45-53.

[128] S.S. Khurana. Pointwise compactness and measurability // Pacific J. Math. 1979. V. 83. № 2. P. 387-391.

[129] S.S. Khurana, J. Vielma. Weak sequential convergence and weak compactness in spaces of vector-valued continuous functions // J. Math. Anal. Appl. 1995. V. 195.№1. P. 251-260.

[130] Д. В. Косыгин, А. А. Минасов, Я. Г. Синай. Статистические свойства спектров операторов Лапласа-Бельтрами на поверхностях Лиувилля // УМН. 1993. Т. 48. №4. C. $3-130$.

[131] A. T.-M. Lau. Finite-dimensional invariant subspaces for a semigroup of linear operators // J. Math. Anal. Appl. 1983. V. 97. № 2. P. 374-379.

[132] A. T.-M. Lau. Amenability of semigroups // The Analytical and Topological Theory of Semigroups. Berlin: de Gruyter, 1990. P. 313-334. (de Gruyter Exp. Math. V. 1.)

[133] A. T.-M. Lau, V. Losert. Complementation of certain subspaces of $L_{\infty}(G)$ of a locally compact group // Pacific J. Math. 1990. V. 141. № 2. P. 295-310.

[134] A. T.-M. Lau, V. Losert. Ergodic sequences in the Fourier-Stieltjes algebra and measure algebra of a locally compact group // Trans. Amer. Math. Soc. 1999. V. 351. №1. P. $417-428$.

[135] A. T.-M. Lau, P. Milnes, J.S. Pym. Compactifications of locally compact groups and closed subgroups // Trans. Amer. Math. Soc. 1992. V. 329. №1. P. 97-115.

[136] A. T.-M. Lau, P. Milnes, J.S. Pym. Flows on invariant subsets and compactifications of a locally compact group // Colloq. Math. 1998. V. 78. № 2. P. 267-281.

[137] A. T.-M. Lau, P. Milnes, J. Pym. On the structure of minimalleft ideals in the largest compactification of a locally compact group // J. London Math. Soc. (2). 1999. V. 59. №1. P. $133-152$.

[138] A. T.-M. Lau, J. C.S. Wong. Weakly almost periodic elements in $L_{\infty}(G)$ of a locally compact group // Proc. Amer. Math. Soc. 1989. V. 107. № 4. P. 1031-1036.

[139] J. D. Lawson. Joint continuity in semitopological semigroups // Illinois J. Math. 1974. V. 18. P. $275-285$.

[140] Б.М. Левитан. Очерк истории теории почти периодических функций // Историко-математические исследования. 1980. Т. 25. С. 156-166.

[141] Б. М. Левитан, В.В.Жиков. Почти периодические функции и дифференциальные уравнения. М.: Изд-во Моск. ун-та, 1978.

[142] M. Lin, J. Olsen. Besicovitch functions and weighted ergodic theorems for LCA group actions // Convergence in Ergodic Theory and Probability (Columbus, OH, 1993). Berlin: de Gruyter, 1996. P. 277-289. (Ohio State Univ. Math. Res. Inst. Publ. V. 5.)

[143] M. Lin, A. Tempelman. Averaging sequences and modulated ergodic theorems for weakly almost periodic group representations // J. Anal. Math. 1999. V. 77. P. 237-268.

[144] E. Lindenstrauss. Pointwise theorems for amenable groups // Invent. Math. 2001. V. 146. № 2. P. 259-295.

[145] L. H. Loomis. The spectral characterization of a class of almost periodic functions // Ann. of Math. (2). 1960. V. 72. P. 362-368.

[146] L. G. Lucht. Recurrent and almost-periodic sequences // Arch. Math. (Basel). 1997. V. 68. №1. P. 22-26

[147] Ю. И. Любич. Введение в теорию банаховых представлений групп. Харьков: Вища школа, 1985. 
[148] Yu. I. Lyubich, Vũ Quoc Phóng. Asymptotic stability of linear differential equations in Banach spaces // Studia Math. 1988. V. 88. №1. P. 37-42.

[149] W. Maak. Fastperiodische Funktionen. Berlin: Springer-Verlag, 1967. (Grundlehren Math. Wiss. V. 61.)

[150] A. A. Markov. Stabilität im Liapounoffschen Sinne und Fastperiodizität // Math. Z. 1933. V. 36. P. 708-738.

[151] M. Mayer. Strongly mixing groups // Semigroup Forum. 1997. V. 54. №3. P. 303-316.

[152] M. Mayer. Asymptotics of matrix coefficients and closures of Fourier-Stieltjes algebras // J. Funct. Anal. 1997. V. 143. № 1. P. 42-54.

[153] M. G. Megrelishvili. Fragmentability and continuity of semigroup actions // Semigroup Forum. 1998. V. 57. №1. P. 101-126.

[154] M. Megrelishvili. Generalized Heisenberg groups and Shtern's question // Georgian Math. J. 2004. V. 11. № 4. P. 775-782.

[155] M.G. Megrelishvili, V.G. Pestov, V. V. Uspenskij. A note on the precompactness of weakly almost periodic groups // Nuclear Groups and Lie Groups (Madrid, 1999). Lemgo: Heldermann, 1985. P. 209-216. (Res. Exp. Math. V. 24.)

[156] J. Mendoza. Necessary and sufficient conditions for $C(X ; E)$ to be barrelled or infrabarrelled // Simon Stevin. 1983. V. 57. №1-2. P. 103-123.

[157] P. Milnes. On vector-valued weakly almost periodic functions // J. London Math. Soc. (2). 1980. V. 22. №3. P. 467-472.

[158] P. Milnes. Almost periodic functions on semitopological semigroups // Rocky Mountain J. Math. 1982. V. 12. №2. P. 265-269.

[159] P. Milnes. Semigroup compactifications of direct and semidirect products // Canad. Math. Bull. 1983. V. 26. №2. P. 233-239.

[160] P. Milnes. The Bohr almost periodic functions do not form a linear space // Math. Z. 1984. V. 188. №1. P. 1-2.

[161] P. Milnes. Minimal and distal functions on semidirect products of groups. II // Math. Scand. 1986. V. 58. № 2. P. 215-226.

[162] P. Milnes. On Bohr almost periodicity // Math. Proc. Cambridge Philos. Soc. 1986. V. 99. №3. P. 489-493.

[163] P. Milnes. Minimal and distal functions on semidirect products of groups // Colloq. Math. 1988. V. 55. № 2. P. 267-275.

[164] P. Milnes. Bohr almost periodicity and functions of dynamical type // Mat.-Fys. Medd. Danske Vid. Selsk. 1989. V. 42. №3. P. 113-118.

[165] P. Milnes. On distal and equicontinuous compact right topological groups // Int. J. Math. Math. Sci. 1994. V. 17. № 2. P. 379-388.

[166] P. Milnes, J.S. Pym. Function spaces on semitopological semigroups // Semigroup Forum. 1980. V. 19. №4. P. 347-354.

[167] M.R. Miri, M. A. Pourabdollah. Minimally weakly almost periodic semigroups // Far East J. Math. Sci. 1998. Special Volume. Part II. P. 191-202.

[168] R. T. Moore. Measurable, continuous and smooth vectors for semigroup and group representations // Mem. Amer. Math. Soc. 1968. V. 78.

[169] Н. Г. Мощевитин. Дифференциальные уравнения с почти и условно периодическими коэффициентами: возвращаемость и приводимость // Матем. заметки. 1998. Т. 64. № 2 . C. $229-237$.

[170] G. Muni. Functions of $\mathfrak{S}$ almost periodic with respect to a homeomorphic group // Boll. Unione Mat. Ital. (B) (5). 1980. V. 17. №1. P. 232-243.

[171] M. A. Naimark, A. I. Štern [Shtern]. Theory of Group Representations. New York: Springer-Verlag, 1982.

[172] I. Namioka. Separate continuity and joint continuity // Pacific J. Math. 1974. V. 51. P. $515-531$.

[173] I. Namioka, R. R. Phelps. Banach spaces which are Asplund spaces // Duke Math. J. 1975. V. 42. №4. P. 735-750. 
[174] K.-H. Neeb, D. Pickrell. Supplements to the papers entitled: "On a theorem of S. Banach" by Neeb and "The separable representations of $U(H)$ " by Pickrell // J. Lie Theory. 2000. V. 10. №1. P. 107-109.

[175] В. Ф. Осипов. Почти периодические функции Бора-Френеля. СПб.: СПбГУ, 1992.

[176] B. K. Pal, S. N. Mukhopadhyay. The Cesàro-Denjoy-Bochner scale of almost periodicity // Anal. Math. 1991. V. 17. №4. P. 307-331.

[177] A. L. T. Paterson. Amenability. Providence, RI: Amer. Math. Soc., 1988.

[178] V. Pestov. Independence of almost periodicity upon the topology of the acting group // Topology Appl. 1998. V. 90. № 1-3. P. 223-225.

[179] А. Пич. Ядерные локально выпуклые пространства. М.: Мир, 1967.

[180] A. R. Plastino, A. Plastino. Tsallis' entropy, Ehrenfest theorem and information theory // Phys. Lett. A. 1993. V. 177. № 3. P. 177-179.

[181] J. Pym. Semigroup structure in Stone-Čech compactifications // J. London Math. Soc. (2). 1987. V. 36. №3. P. 421-428.

[182] D. Remus, F. J. Trigos-Arrieta. The Bohr topology of Moore groups // Topology Appl. 1999. V. 97. № 1-2. P. 85-98.

[183] S. Romanelli. A generalized notion of almost periodicity in connection with vector means and compactifications // Boll. Unione Mat. Ital. B (6). 1986. V. 5. №2. P. 639-659.

[184] S. Romanelli. A Godement-type decomposition for positive definite functions // Harmonic Analysis (Luxembourg, 1987). Berlin: Springer-Verlag, 1988. P. 242-251. (Lecture Notes in Math. V. 1359.)

[185] J. Rosenblatt, W. M. Ruess, D. Sentilles. On the critical part of a weakly almost periodic function // Houston J. Math. 1991. V. 17. №2. P. 237-249.

[186] J. Rosenblatt, Z. Yang. Functions with a unique mean value // Illinois J. Math. 1990. V. 34. № 4. P. 744-764.

[187] W. M. Ruess, W.H. Summers. Integration of asymptotically almost periodic functions and weak asymptotic almost periodicity // Dissertationes Math. (Rozprawy Mat.). 1989. V. 279.

[188] W. M. Ruess, W.H. Summers. Weak almost periodicity and the strong ergodic limit theorem for periodic evolution systems // J. Funct. Anal. 1990. V. 94. № 1. P. 177-195.

[189] W. Ruppert. Compact Semitopological Semigroups: An Intrinsic Theory. Berlin: SpringerVerlag, 1984. (Lecture Notes in Math. V. 1079.)

[190] W. A. F. Ruppert. On signed a-adic expansions and weakly almost periodic functions // Proc. London Math. Soc. (3). 1991. V. 63. № 3. P. 620-656.

[191] C. Ryll-Nardzewski. On fixed points of semigroups of endomorphisms of linear spaces // Proceedings of the Fifth Berkeley Symposium on Mathematical Statistics and Probability (Berkeley, CA, 1965/66). V. II: Contributions to Probability Theory, Part 1. Berkeley: Univ. California Press, 1967. P. 55-61.

[192] K. Sakai. On almost periodic compactifications of semitopological semigroups // Sci. Rep. Kagoshima Univ. 1989. V. 38. P. 39-48.

[193] A. Satyanarayan Rao. On the successive integration of Stepanov-almost periodic functions // Math. Student. 1985. V. 49. №2-4. P. 153-156.

[194] T. Scarr. Topological and Borel compactifications of Polish G-spaces // Topology Appl. 2000. V. 105. № 1. P. 113-119.

[195] Х. Шефер. Топологические векторные пространства. М.: Мир, 1971.

[196] М. Б. Севрюк. Некоторые проблемы теории КАМ: условно-периодические движения в типичных системах // УМН. 1995. Т. 50. №2. С. 111-124.

[197] А. И. Штерн. Stoicheia и платоновы многогранники // Античная культура и современная наука. М.: Наука, 1984. С. 36-43.

[198] A. I. Shtern. Compact semitopological semigroups and reflexive representability of topological groups // Russian J. Math. Phys. 1994. V. 2. № 1. P. 131-132.

[199] A. I. Shtern. Analog of the Eberlein-de Leeuw-Glicksberg theorem and algebras with unique mean value on amenable groups // Russian J. Math. Phys. 2001. V. 8. № 2. P. 246-249. 
[200] А. И. Штерн. Критерии слабой и сильной непрерьвности представлений топологических групп в банаховых пространствах // Матем. сб. 2002. Т. 193. № 9. С. 139-156.

[201] A. I. Shtern. Values of invariant means, left averaging, and criteria for a locally compact group to be amenable as discrete group // Russian J. Math. Phys. 2003. V. 10.№ 2 . P. $185-198$.

[202] A. I. Shtern. Representations of topological groups in locally convex spaces: continuity properties and weak almost periodicity // Russian J. Math. Phys. 2004. V. 11. № 1. P. 81-108.

[203] A. I. Shtern. Weakly almost periodic representations of topological groups in Mackey locally convex spaces // Adv. Stud. Contemp. Math. 2005. V. 10. № 2 (to appear).

[204] А. И. Штерн. Топологические группы с конечными алгебрами фон Неймана типа I // Матем. сб. 2005. Т. 196. №3. С. 143-160.

[205] М. А. Шубин. Спектральная теория и индекс эллиптических операторов с почти периодическими коэффициентами // УМН. 1979. Т. 34. № 2. С. 95-135.

[206] C. Stegall. The Radon-Nikodým property in conjugate Banach spaces // Trans. Amer. Math. Soc. 1975. V. 206. P. 213-223.

[207] C. Stegall. The duality between Asplund spaces and spaces with the Radon-Nikodým property // Israel J. Math. 1978. V. 29. №4. P. 408-412.

[208] S. Stoiński. On compactness in variation of almost periodic functions // Demonstratio Math. 1998. V. 31. № 1. P. 131-134.

[209] M. Talagrand. Some functions with a unique invariant mean // Proc. Amer. Math. Soc. 1981. V. 82. № 2. P. 253-256.

[210] J.L. Taylor. Measure Algebras. Providence, RI: Amer. Math. Soc., 1973. (CBMS Reg. Conf. Ser. Math. V. 16.)

[211] S. Teleman. Sur la représentation linéaire des groupes topologiques // Ann. Sci. École Norm. Sup. (3). 1957. V. 74. P. 319-339.

[212] J.-P. Troallic. Une approche nouvelle de la presque-périodicité faible // Semigroup Forum. 1981. V. 22. № 3. P. 247-255.

[213] J.-P. Troallic. Semigroupes semitopologiques et presque-périodicité // Recent Developments in the Algebraic, Analytical, and Topological Theory of Semigroups (Oberwolfach, 1981). Berlin: Springer-Verlag, 1983. P. 239-251. (Lecture Notes in Math. V. 998.)

[214] E. R. van Kampen, A. Wintner. On the almost periodic behavior of multiplicative number-theoretical functions // Amer. J. Math. 1940. V. 62. P. 613-626.

[215] W. A. Veech. Almost automorphic functions on groups // Amer. J. Math. 1965. V. 87. P. $719-751$.

[216] W. A. Veech. Topological dynamics // Bull. Amer. Math. Soc. 1977. V. 83. №5. P. $775-830$.

[217] K. Venkataraman. Boolean valued almost periodic functions: existence of the mean // J. Indian Math. Soc. (N.S.). 1979. V. 43. № 1-4. P. 275-283.

[218] E. Vesentini. Spectral properties of weakly asymptotically almost periodic semigroups // Adv. Math. 1997. V. 128. № 2. P. 217-241.

[219] J. von Neumann. Almost periodic functions in a group. I // Trans. Amer. Math. Soc. 1934. V. 36. № 2. P. 445-492.

[220] J. von Neumann. On complete topological groups // Trans. Amer. Math. Soc. 1935. V. 37. P. 1-20.

[221] B. Weiss. Single Orbit Dynamics. Providence, RI: Amer. Math. Soc., 2000 (CBMS Reg. Conf. Ser. Math. V. 95.).

[222] H. Weyl. Almost periodic invariant vector sets in a metric vector space // Amer. J. Math. 1949. V. 71. P. $178-205$.

[223] T.S. Wu. Left almost periodicity does not imply right almost periodicity // Bull. Amer. Math. Soc. 1966. V. 72. P. 314-316.

[224] G. Xu. Herz-Schur multipliers and weakly almost periodic functions on locally compact groups // Trans. Amer. Math. Soc. 1997. V. 349. №6. P. 2525-2536.

[225] Xu, Q.. Representations and compactifications of locally compact groups // Math. Japon. 2000. V. 52. №2. P. 323-329. 
[226] D. Yost. Asplund spaces for beginners // Acta Univ. Carolin. Math. Phys. 1993. V. 34. № 2. P. 159-177.

[227] N. J. Young. Periodicity of Functionals and Representations of Normed Algebras on Reflexive Spaces // Proc. Edinburgh Math. Soc. (2). 1976/77. V. 20. № 2. P. 99-120.

[228] S. D. Zaidman. Almost-periodic Functions in Abstract Spaces. Boston, MA: Pitman, 1985.

[229] C.-Y. Zhang. Vector-valued means and weakly almost periodic functions // Int. J. Math. Math. Sci. 1994. V. 17. №2. P. 227-237.

[230] C.-Y. Zhang. Vector-valued means and their applications in some vector-valued function spaces // Dissertationes Math. (Rozprawy Mat.). 1994. V. 334. P. 1-35.

[231] C.-Y. Zhang. Integration of vector-valued pseudo-almost periodic functions // Proc. Amer. Math. Soc. 1994. V. 121. № 1. P. 167-174.

[232] C.-Y. Zhang. Vector-valued pseudo almost periodic functions // Czechoslovak Math. J. 1997. V. 47. № 3. P. 385-394.

[233] C.-y. Zhang. Ergodicity and its applications. I. Basic properties // Acta Anal. Funct. Appl. 1999. V. 1. №1. P. 28-39.

[234] C.-y. Zhang. Ergodicity and its applications. II. Averaging method of some dynamical systems // Acta Anal. Funct. Appl. 1999. V. 1. №2. P. 146-159.

[235] В. В. ЖКиков. Об одной задаче Бохнера и фон Неймана // Матем. заметки. 1968. Т. 3. № 5. C. 529-538.

[236] В. В. ЖКиков, С. М. Козлов, О. А. Олейник. Усреднение дифференциальных операторов. М.: Наука, 1993.

[237] В.В.ЖКиков, Е. В. Кривенко. Усреднение сингулярно возмущенных эллиптических операторов // Матем. заметки. 1983. Т. 33. №4. С. 571-582.

Московский государственньй университет им. М. В. Ломоносова

Поступила в редакцию

E-mail: ashtern@member.ams.org

18.08 .2004 